\title{
1 Association of genetic risk factors with cognitive decline: The PATH Through Life Project
}

$3 \quad$ Shea J. Andrews ${ }^{\mathrm{a}}$, Debjani Das ${ }^{\mathrm{a}}$, Nicolas Cherbuin ${ }^{\mathrm{b}}$, Kaarin J. Anstey ${ }^{\mathrm{b}}$ and Simon

${ }_{5}$ a John Curtin School of Medical Research, Australian National University. 131 Garran Rd,

6 Canberra ACT 2601 Australia.

7 besearch School of Population Health, Australian National University. Florey Building 54,

8 Mills Road, Canberra ACT 2601 Australia.

9

${ }_{10}{ }^{*}$ Correspondence to: Shea Andrews, JCSMR, ANU, 131 Garran Rd, Canberra ACT 2601

11 Australia. Tel: +61 26125 2392; Fax: +61 26125 2499; E-mail: shea.andrews@anu.edu.au 
12 Abstract

${ }_{13}$ We examined the association of 28 single nucleotide polymorphisms (SNPs), previously

14

15

26 associated with dementia or cognitive performance, with tests assessing episodic memory, working memory, vocabulary and perceptual speed in 1,689 non-demented older Australians of European ancestry. In addition to testing each variant individually, we assessed the collective association of the 12 risk SNPs for Late-onset Alzheimer's disease (LOAD) using weighted and unweighted genetic risk scores (GRS). Significant associations with cognitive performance were observed for $A P O E *_{\epsilon} 4$ allele, $A B C A 7$-rs3764650, CR1-rs3818361, MS4A4E-rs6109332, BDNF-rs6265, COMT-rs4680, CTNNBL1-rs6125962, FRMD4A-rs17314229, FRMD4A-rs17314229, intergenic SNP chrX-rs12007229, PDE7A-rs10808746, SORL1-rs668387 and ZNF224-rs3746319. Additionally, the weighted GRS was associated with worse performance on episodic memory. The identification of genetic risk factors, that act individually or collectively, may help in screening for people with elevated risk of cognitive decline and for understanding the biological pathways that underlie cognitive decline.

Keywords: Alzheimer's Disease; Cognitive Decline; SNPs; Genetic Risk Scores, Population-Based Study. 


\section{Introduction}

Cognitive differences in the elderly consist of differences in stable, life-long cognitive traits and differences in age-associated cognitive change. For both of these there is significant inter-individual variability in the population (Wilson et al., 2002). Loss of cognitive function due to age-associated cognitive decline is associated with increased difficulties in performing tasks involving memory or rapid information processing and can have a major impact on an individual's quality of life, even in the absence of dementia (Boyle et al., 2012; Kobayashi et al., 2014; Tucker-Drob, 2011; Wilson et al., 2013; Yam and Marsiske, 2013; Zahodne et al., 2013). Identifying factors that predispose individuals to a faster rate of cognitive decline is an important step for developing intervention and treatment strategies aimed at maintaining cognitive health.

Genetic factors likely contribute to the inter-individual variability observed in cognitive decline, with common genetic variants estimated to account for between $40-50 \%$ of the variability associated with general cognitive functioning in later life and $24 \%$ of the variability in lifetime cognitive change (Davies et al., 2011; Deary et al., 2012). To date the majority of genetic research on cognitive decline has focused on candidate genes that have been previously associated with age-related disease, traits or mechanisms (Payton, 2009; Harris and Deary, 2011), and particularly with genes related to neurotransmitters, neurotrophins, cognitive function and neurodegenerative disease. Two of the most widely studied such genes are COMT, which encodes the neurotransmiter catechol-O-methyl transferase, and $B D N F$, which encodes the neurotrophin brain-derived neurotrophic factor. Functional variants in these genes have been primarily associated with decline in executive functioning and memory, respectively, although results are inconsistent (Payton, 2009). Late-onset Alzheimer's disease (LOAD) susceptibility genes are also good candidates for association with cognitive decline as the pathological features of LOAD progress to varying degrees in individuals without dementia or cognitive impairment and are associated with non-clinical cognitive 
decline (Boyle et al., 2013b; Savva et al., 2009). This cross-over effect is exemplified by the $A P O E *_{\epsilon 4}$ allele, which confers the largest known genetic risk for LOAD, approximately 2-3 times and 10-12 times for heterozygotes and homozygotes respectively (Farrer et al., 1997). The APOE genotypes is also associated with specific effects on the cognitive domains of episodic memory, executive functioning, perceptual speed and global cognitive ability (Wisdom et al., 2011).

Despite the publication of numerous genetic associations with cognitive decline, the variants identified typically explain a very small fraction of the phenotypic variability and many remain to be replicated. Furthermore, failure to replicate an initial positive result is common due to differences in participant characteristics (e.g. baseline education, mean age, gender and ethnicity) and methodologies (e.g. sample size, duration of the study, number of follow-ups, population stratification, variation in classification and cognitive measures) (Payton, 2009).

Here we investigate the association between selected genetic risk factors with cognitive decline in a longitudinally followed community-based cohort of 1,689 older adults without dementia who have undergone comprehensive cognitive testing. First, we investigate whether 12 SNPs from the top replicated LOAD associated genes (Morgan and Carrasquillo, 2013; Supplementary Tables 1 and 2) are individually, or collectively as a genetic risk score (GRS), associated with cognitive decline. Second, we investigate whether 16 SNPs, previously associated with either dementia or cognition (Table 1 and Supplementary Table 2), are also associated with cognitive decline.

\section{Methods}

\subsection{Participants}

Participants were recruited randomly from the electoral rolls (registration is a legal requirement 
for Australian Citizens) of Canberra and Queanbeyan into the Personality and Total Health (PATH) Through Life Project. PATH consists of three cohorts 20-24 (20+), 40-44 (40+) and 60-64 (60+) years at baseline, who have participated in a large longitudinal community survey of health and wellbeing in adults, the background and procedures for which have been described in detail elsewhere (Anstey et al., 2012). Written informed consent was obtained from all participants and approval for the study was obtained from the Human Research Ethics Committee of The Australian National University.

The $60+$ cohort is the focus of this study. Individuals were assessed at 4-year intervals for a period of 8 years with interviews conducted in 2001-2002 $(\mathrm{n}=2,551), 2005-2006(\mathrm{n}=$ $2,222)$ and 2009-2010 $(\mathrm{n}=1,973)$. Individuals were excluded from further analysis based on the following criteria: attendance at only one interview $(\mathrm{n}=309)$, no genomic DNA available for genotyping $(\mathrm{n}=185), A P O E{ }^{*} \epsilon 2 /{ }^{*} \epsilon 4$ genotype $(\mathrm{n}=60$; to avoid the conflation of ${ }^{*} \epsilon 2$ protective and ${ }^{*} \epsilon 4$ risk effect), non-European ancestry $(\mathrm{n}=110)$, probable dementia at any wave (Mini Mental State Examination score $<24$ (Folstein et al., 1975), self-reported medical history of epilepsy, stroke, transient ischaemic attack, brain tumour or brain infection $(\mathrm{n}=327)$. Missing values, which can reduce power and result in biased estimates, were imputed for the covariate 'Education' (total years of education) using random forests via the 'missForest' package available in R (Stekhoven and Bühlmann, 2012) ( $\mathrm{n}=139)$. This left a final sample of 1,689 individuals. At baseline, the individuals retained in the final sample had on average of 0.69 more years of education and scored 0.74 points higher on the MMSE than those excluded (Table 1).

\subsection{Cognitive Assessment}

All participants were assessed at baseline and at each subsequent interview for the following five cognitive abilities: perceptual speed was assessed using the Symbol Digit Modalities Test, which asks the participant to substitute as many digits for symbols as possible in 
90s (Smith, 2002); episodic memory was assessed using the immediate recall and delayed recall of the first trial of the California Verbal Learning Test, which involves recalling a list of 16 nouns (Delis et al., 1987); working memory was assessed using the Digit Span Backward from the Wechsler Memory Scale, which presents participants with series of digits increasing in length at the rate of one digit per second and asks them to repeat the digits backwards (Wechsler, 1945); and vocabulary was assessed with the Spot-the-Word Test, which asks participants to choose the real words from 60 pairs of words and nonsense words (Baddeley et al., 1993). (Supplementary Tables 5 and 6).

\subsection{Genotyping}

Sixty-four single nucleotide polymorphisms (SNPs) were selected for genotyping based on previous associations with dementia, cognition, neuroanatomical differences and blood pressure (Supplementary Table 2). Genomic DNA was extracted from cheek swabs ( $\mathrm{n}=$ 4,597) using Qiagen DNA blood kits or from peripheral blood leukocytes $(\mathrm{n}=64)$ using QIAamp DNA 96 DNA blood kits.

Pre-amplification of the targeted loci was performed using the TaqMan PreAmp Master Mix Kit (Life Technologies). Each reaction included 2.5 $\mu \mathrm{L}$ TaqMan PreAmp Master Mix $(2 \times), 1.25 \mu \mathrm{L}$ Pre-amplification Assay Pool, $0.5 \mu \mathrm{L} \mathrm{H}_{2} 0$ and $1.2 \mu \mathrm{L}$ genomic DNA. These reactions were incubated in a Biorad thermocycler for 10 min at $95^{\circ} \mathrm{C}$, followed by 12 cycles of $95^{\circ} \mathrm{C}$ for $15 \mathrm{sec}$ and $60^{\circ} \mathrm{C}$ for $4 \mathrm{~min}$, and then incubated at $99.9^{\circ} \mathrm{C}$ for 10 minutes. The PreAmplified products were then held at $4^{\circ} \mathrm{C}$ until they were diluted 1:20 in $1 \times$ TE buffer and then stored at $-20^{\circ} \mathrm{C}$ until use.

$2.5 \mu \mathrm{L}$ diluted pre-amplified products was mixed with $2.5 \mu \mathrm{L}$ TaqMan OpenArray Master Mix. The resulting samples were dispensed using the OpenArray ${ }^{\circledR}$ AccuFill ${ }^{\mathrm{TM}}$ System onto OpenArray plates with each plate containing 48 samples and 64 SNP assays per sample. 
The QuantStudio $^{\text {TM }}$ 12K Flex instrument (Applied Biosystems, Carlsbad, California) was used to perform the real time PCR reactions on the loaded OpenArray plates. The fluorescence emission results were read using the OpenArray ${ }^{\circledR}$ SNP Genotyping Analysis software v1 (Applied Biosystems) and the genotyping analysis was performed using TaqMan ${ }^{\circledR}$ Genotyper v1.3, using the autocalling feature. Participant-specific quality controls included filters for genotype success rate $(>90 \%)$, genotype-derived gender concordant with reported gender and sample provenance error assessed via pairwise comparisons of genotype calls between all samples to identify samples with $>90 \%$ similarity. Samples that were flagged in the initial quality control checks were repeated, those that still failed quality control were excluded. SNP-specific filters included genotype call rate $(>90 \%)$ and Hardy-Weinberg equilibrium $(p>0.001)$ assessed using an exact test with the PLINK toolkit (Purcell et al., 2007).

For this study, data for 28 of the 64 genotyped SNPs was extracted based on a priori hypotheses (Supplementary Table 1). These SNPs have being previously identified as being associated with dementia or cognition through GWAS or candidate gene studies (Supplementary Table 2) and consist of 12 SNPs that have been highly replicated as being associated with LOAD and an additional 16 SNPs whose associations are ambiguous and are in need of further replication. Genotyping of the PATH sample for $A P O E$ variants was performed separately and has been described previously (Jorm et al., 2007). The SNPs were in Hardy-Weinberg equilibrium and genotype frequencies are presented in Supplementary Tables 3 and 4.

\subsection{Data Preparation and Statistical Analysis}

Data were analysed in the R Statistical Computing environment (R Core Team, 2013).

We created an index for episodic memory using the average scores of the immediate and delayed recall tasks. To allow for comparison across all cognitive tasks, the tests scores for each cognitive task at all three waves were transformed into $\mathrm{Z}$ scores $(\mathrm{M}=0, \mathrm{SD}=$ 1), using the baseline means and standard deviations. Higher test scores indicate better 
cognitive function.

Genetic dominance was assumed for previously reported risk alleles (Bertram et al., 2007) for LOAD GWAS SNPs, and for minor alleles (alleles with the lowest frequency in the population) of the 16 additional SNPs. The $A P O E *^{*} \epsilon 4$ and ${ }^{*} \epsilon 2$ alleles were assumed to be dominant to the $\epsilon 3$ allele. For $A P O E$ participants were classified as either APOE $*_{\epsilon} 4+$ $\left({ }^{*} \epsilon 4 /{ }^{*} \epsilon 4+{ }^{*} \epsilon 4 /{ }^{*} \epsilon 3\right),{ }^{*} \epsilon 2+\left({ }^{*} \epsilon 2 /{ }^{*} \epsilon 2+{ }^{*} \epsilon 2 /{ }^{*} \epsilon 3\right)$ or ${ }^{*} \epsilon 3\left({ }^{*} \epsilon 3 /{ }^{*} \epsilon 3\right)$. Because we wanted to assess the independent contributions of ${ }^{*} \epsilon 4$ and ${ }^{*} \epsilon 2$ to cognitive decline, those with the $*_{\epsilon} 2 /{ }^{*} \epsilon 4$ genotype were excluded.

Three genetic risk scores (Che and Motsinger-Reif, 2013) were calculated using the LOAD GWAS SNPs: 1) a simple count genetic risk (SC-GRS): the sum of all risk alleles across all loci; 2) an odds-ratio weighted genetic risk score (OR-GRS): the sum of all risk alleles across all loci, weighted by effect size of the risk allele on AD, as reported in the AlzGene Database (Bertram et al., 2007); 3) an explained variance-weighted genetic risk score (EV-GRS): the sum of all risk alleles across all loci, weighted by minor allele frequency and effect size on $\mathrm{AD}$, as reported in the AlzGene Database. For all genetic risk scores a higher value indicates greater risk. The MAF and OR used to derived the GRS are presented in Supplementary Table 1. Individuals missing any genetic data $(\mathrm{n}=69)$ were excluded from the analysis.

Linear mixed effect models (LMM) with maximum likelihood estimation and subject-specific random slopes and intercepts were used to assess the effect of predictors on change in cognitive test scores over time. Age, centered on mean age at baseline, was used as an indicator of time in the study. The predictor variables included in the analysis were the individual SNPs or the three GRS's: SC-GRS, OR-GRS and EV-GRS. Covariates used in the models included, sex, education and for individual SNP models APOE genotype. LMM's were estimated using the R package 'Ime4' (Bates et al., 2014) and $F$ and $p$ values were estimated using Satterthwaite-type approximation were used to determine the statistical significance 
of the fixed effects. To evaluate if the random slopes were significantly different from 0 and to determine if there was residual variability in the rate of change that could be explained by predictor variables, LMM's that included random slopes were compared to models that did not include random slopes using parametric bootstrap methods where 1000 simulations of the likelihood ratio test statistic were generated ( $\mathrm{R}$ package 'pbkrtest', Halekoh and Højsgaard, 2012). For each SNP and GRS we compared the model fit of the full model with the covariates-only model to evaluate if there was an overall effect of the SNP or GRS on cognitive decline. Model fit was assessed using a Kenward-Rodger approximation for F-tests (R package 'pbkrtest', Halekoh and Højsgaard, 2012). Two $R^{2}$ statistics were calculated to quantify, 1) the proportion of outcome variation explained by the fixed factors (marginal $R^{2}$ ) and 2) the amount of outcome variation explained by the fixed and random factors (conditional $R^{2}$; Johnson, 2014; Nakagawa and Schielzeth, 2013; R package 'MuMIn' Barton and Barton, 2013). Additionally, we performed a secondary analysis in which changes in the rate of cognitive decline by genotype were estimated separately for participants who were classified as cognitive impaired (CI) at wave 3 if they scored $<=27$ on the MMSE $(\mathrm{n}=118)$ and those classified as cognitively normal $(\mathrm{CN}, \mathrm{n}=1340)$. For the secondary analysis, LMM's were performed with the inclusion of the additional terms for a time by cognitive status and separate time by genotype interactions for the CI and CN classifications. We did not adjust for multiple comparison as strong a priori evidence for all our hypothesis based on previous findings for LOAD and cognitive decline was available; a $P$-value $<0.05$ was considered statistically significant.

\section{Results}

\subsection{Population characteristics of the PATH cohort}

General demographics of the PATH cohort are presented in 1. Linear Mixed Models 1-3 in Supplementary Table 7-9 show the average rate of change for each cognitive test. Random slopes for all cognitive tests scores were significantly different from 0 , indicating that there 
was sufficient variability in the rate of change between participants thus allowing potential genetic predictors of this change to be tested (bootstrap $P$ value: Episodic Memory $=$ 0.04; Digits backwards $=0.01 ;$ Spot-the-Word test $=0.0001 ;$ Symbol digits modalities test $=0.01)$. Significant change in test scores over time was observed for all cognitive tests except Digits Backwards. In model 2, participants experienced an overall decline in test scores for Episodic memory and Symbol digits Modalities Test, and an increase in test scores for Spot-the-Word. 'Time' explained 57-89\% of outcome variation for the entire model. The covariates in model 3 improved the model fit for all cognitive tests and explained $7-21 \%$ of the outcome variation in the fixed effects, although they did not explain any additional random effect variation for the entire model (Supplementary Tables 9).

\subsection{Main effects of LOAD GWAS SNPs}

There was a significant improvement in model fit for various cognitive tests after the introduction of the $A P O E, A B C A 7, C R 1$ and $M S_{4} A 4 E$ SNPs into their respective models. $A P O E \epsilon 4+$ was associated with a greater rate of decline in Episodic memory and the association remained unchanged when $A P O E \epsilon 3 / \epsilon 4$ heterozygotes were assessed separately from $A P O E \in 4$ homozygotes and $A P O E \in 2$ carriers; $A B C A 7$-rs3764650-G was associated with a lower initial status at baseline in Episodic memory test scores; CR1-rs3818361-A was associated with a greater rate of decline in Episodic memory and; $M S_{4} A 4 E$-rs670139-T was associated with a higher baseline Spot-the-Word Test score and a slower decline in Episodic memory test scores. The group differences resulted in a small increase in the marginal $R^{2}$ ranging from 0.001 to 0.002, though there was no increase in the conditional $R^{2}$ statistics. Table 2 , Supplementary Tables 10-21.

The remaining SNPs (BIN1, CD2AP, CD33, CLU, EPHA1, MS4A4A, MS4A6A and PICALM) were not significantly associated with baseline status or rate of change for any of cognitive tests.

In the secondary analysis assessing the rate of cognitive decline separately for participants 
who were classified as CI (supplementary Table 44), the APOE $\epsilon 4+$ was associated with a faster rate of decline in Episodic memory for CI and CN participants, with a steeper decline observed in CI participants, and a reduced rate of decline in Digits Backwards test scores in CN participants; $A B C A$ \%-rs3764650-G was associated with a faster rate of decline in Digits backwards tests scores in CI participants and; EPHA1-rs11767557-T was associated with as faster rate of decline SDMT tests in CI participants.

\subsection{Effect of Genetic Risk Scores}

The equally-weighted SC-GRS has an approximately normal distribution in the PATH Cohort $($ Figure 1 ; mean $=10.5$; range $=3-18)$. The bimodal distribution and long upper tails of the weighted OR- and EV-GRS reflect the strong effect of $A P O E$ relative to other loci $($ Figure $1 ;$ mean $=1.47$; range $=-0.7-4.5 \&$ mean $=0.92 ;$ range $=-0.1-2.4$ respectively $)$. The SC-GRS was not significantly associated with either initial status at baseline or rate of change for any of the cognitive tests. There was a significant improvement in model fit for Episodic memory for both the OR- and EV-GRS, with higher OR- and EV-GRS being associated with a greater rate of decline in cognitive performance. These associations resulted in a small increase in the amount of explained variation in the fixed effects, in comparison to that explained by time and the covariates, of 0.001 and 0.002 for the OR-GRS and EV-GRS respectively though there was no increase in the conditional $R^{2}$ statistics. (Table 2, supplementary Tables 22-24). The OR- and EV-GRS were not associated with cognitive performance when the APOE allele was excluded (supplementary Tables 25-27). In the secondary analysis (supplementary Table 44), the OR- and EV-GRS were associated with a faster rate of decline in episodic memory in both CI and CN participants, with a steeper decline observed in CI participants.

\subsection{Main effects of SNPs associated with dementia or cognition}

Statistics for the models introducing the additional dementia and cognition SNPs are shown 
in Table 3. See Supplementary Tables 28-43 for full models with random and fixed effects. A significant improvement in model fit was observed for a number of cognitive tests after the introduction of the following SNPs: BDNF, COMT, FRMD4A-rs7081208, Intergenic chrX, PDE $7 A$ and ZNF224 into their respective models. In these models significant parameter estimates were observed. $B D N F$-rs6265-T was associated with a lower baseline Digits Backwards tests scores while COMT-rs4680-A with a greater rate of decline in Episodic memory test scores. FRMD4A-rs7081208-A was associated with a lower baseline score as well as a slower rate of decline in Digits Backwards test scores and with higher baseline scores, but a greater rate of decline in Spot-the-Word test scores. Intergenic-rs12007229-A was associated with a greater rate of decline in Episodic memory test scores. PDE 7 A-rs10808746-A was associated with a slower rate of decline in Symbol Digits Modalities test scores; ZNF224-rs3746319-A associated with a higher baseline Spot-the-Word Test scores.

Statistically significant parameter estimates in the absence of improvement in model fit were also observed, with CTNNBL1-rs6125962-C associated with a reduced rate of decline in Episodic memory test scores; FRMD4A-rs17314229-T was associated with a greater rate of decline in digits backwards test scores; $P D E 7 A$-rs10808746-A was associated with lower digits backwards test baseline scores and a slower rate of decline in Symbol Digits Modalities test scores; SORL1-rs668387-T was associated with higher Spot-the-Word Test scores at baseline.

These significant associations result in a small increase in explained variation in the fixed effects ranging from 0.0003 to 0.003 , though no increase in the conditional $R^{2}$.

In the secondary analysis assessing the rate of cognitive decline separately for participants who were classified as CI or CN are presented in supplementary Table 45). BDNF-rs6265-T, CETP-rs5882-G, MTHFD1L-rs11754661-A, CTNNBL1-rs6125962-C, FRMD4A-rs17314229-T, PAICS-rs11549976-A and PEDYA-rs10808746-A were associated with rate of change in participants classifed as CI. COMT-rs4680-A, FRMD4A-rs7081208-A and Intergenic-rs12007229-A were associated with rate of change in participants who were classified as CN. 


\section{Discussion}

In this study we investigated the association between common genetic variants that have been previously reported to be associated with LOAD, dementia or cognition with change in episodic memory, working memory, vocabulary and perceptual speed. The top LOAD GWAS SNPs were primarily associated with cognitive performance in episodic memory. This is likely indicative of their role in Alzheimer's disease, as progressive deficits in episodic memory that begin early on in the disease course are one of its defining features (Dubois et al., 2010). Associations with rate of change in cognitive performance were observed for $A P O E, C R 1, M S_{4} A 4 E$, while $A B C A 7$ was associated with baseline cognitive performance. The direction of the effect for $A P O E, C R 1$, and $A B C A 7$ was as expected, however for the $M S 4 A 4 E$ the $\mathrm{AD}$ risk allele was associated with a protective effect on episodic memory, and the same trend was also observed for the SNPs $M S_{4} A 6 A$ and $M S_{4} A 4 A$, though they were not significant. However, the parameter estimates for the effect of these SNPs on change in cognitive abilities ranged from $-0.9 \%$ to $0.9 \%$ over four years, while the increase in the marginal $R^{2}$ statistics after inclusion of the genetic predictors ranged from 0.001 to 0.002, emphasising that the effect of individual SNPs on cognition are extremely small.

Additionally, we constructed three genetic risk scores to investigate the combined effect of the LOAD risk SNPs on cognitive decline. An odds ratio weighted GRS and a novel combined odds ratio and minor allele frequency weighted GRS were significantly associated with steeper rate of cognitive decline in episodic memory. The EV-GRS takes into account that within the same OR disease risk can vary depending on the risk allele frequencies and has been shown to be more robust approach for identifying associations in the presence of potential genetic interactions, linkage disequilibrium and false positive predictors (Che and Motsinger-Reif, 2013). An unweighted GRS was not associated with cognitive performance. This latter score utilised a simple count of the number of risk allele per individual and does 
not take into account the varying effect sizes among the LOAD risk SNPs. The lack of significant associations with the SC-GRS, in contrast to the weighted methods, indicates that the significant associations observed for the OR- and EV-GRS can be attributed to the dominant role of the $A P O E{ }^{*} \epsilon 4$ allele, which was further confirmed when $A P O E$ was excluded from the GRS.

These results are similar to those of several comparable candidate gene (Carrasquillo et al., 2015; Engelman et al., 2013) and GWAS based (Davies et al., 2014; De Jager et al., 2012; Zhang and Pierce, 2014) studies, that reported a lack of robust associations between cognitive decline and non-APOE LOAD risk genes. These previous studies have only identified suggestive evidence for CR1 (De Jager et al., 2012; Vivot et al., 2015), CLU (Mengel-From et al., 2013) BIN1 (Vivot et al., 2015) and PICALM (Zhang and Pierce, 2014). However, when examining genetic associations with general cognitive function in middle and older age in a large meta-analysis of 31 studies $(\mathrm{n}=53,949)$ no associations were observed with any of these LOAD risk genes, though two other AD related genes, $M E F 2 C$ and $A B C G 1$, were associated (Davies et al., 2015). Furthermore, when the non-APOE LOAD GWAS risk loci were assessed collectively as a GRS weighted by their estimated OR's, no associations were observed with cognitive decline (De Jager et al., 2012; Carrasquillo et al., 2015), though after inclusion of $A P O E$ the GRS did reach significance (Carrasquillo et al., 2015; Vivot et al. (2015)). An alternative approach using a polygenic risk score of all LOAD associated variants, not just the top associated loci, found no association with cognitive ability in later life or with age-related cognitive change (Harris et al., 2014). Investigating interactions between environmental and lifestyle factors and GRS may provide more promising results, with a higher GRS composed of $A P O E, C L U, C R 1$ and PICALM, while not independently associated with cognitive decline was shown to exacerbate the deleterious effects of type 2 diabetes on cognitive decline (McFall et al., 2015). 
Alzheimer's related genes may be associated with cognitive decline in subjects who are in the preclinical stages of dementia and who, if followed for long enough, might eventually develop dementia. We also assessed the effect of the LOAD risk SNPs separately for those who were classified as cognitively impaired according to MMSE at wave 3 and observed faster rates of decline associated with the $A B C A 7$ risk allele and reduced rates of decline associated with the EPHA1 risk allele In previous studies, variants in $A B C A 7, E P H A 1$ (Carrasquillo et al., 2015) and $C L U$ (Thambisetty et al., 2013) have been observed to be associated with cognitive decline in subjects who eventually converted to dementia, but not in individuals who remained cognitively normal throughout the study. This also suggests that associations observed between LOAD risk genes and cognitive decline could be due to individuals who are in the preclinical stages of Alzheimer's disease and that the retrospective removal of these individuals could attenuate the observed associations (Knight et al., 2014; Davies et al., 2015). However, removing individuals who are in the preclinical stages of disease in the early analytic stages of a study is likely to be difficult due to the long and asymptomatic nature of the preclinical stages of LOAD.

The above findings, and those of previous studies, suggest that the added predictive value of the top LOAD SNPs for cognitive decline in non-demented individuals may be limited. This is consistent with polygenic models of cognitive decline indicating that there is a large number of variants with modest effects sizes rather than a few variants with large or moderate effect sizes. Additionally, this is consistent with indices of LOAD pathology (amyloid and neurofibrillary tangles) that only explain $30 \%$ of observed variance in cognitive decline, and cerebrovascular (macro and micro infarcts) and Lewy body disease neuropathologies explaining an additional 10\% of variation (Boyle et al., 2013a). This is consistent with the notion that while LOAD pathology is important in the development of cognitive decline, it occurs in conjunction with other pathological features that are observed in brain ageing. As such, the cognitive deficits observed in brain ageing are unlikely to be due to an isolated 
pathological feature, but the interaction between multiple neuropathologies (Keller, 2006).

This highlights the need to investigate additional genetic variants in addition to those associated with AD.

For the remaining 16 SNPs investigated, which were previously associated with dementia or cognitive performance, we observed associations with a increased rate of decline in cognitive performance for the minor alleles of COMT and Intergenic chrX, while for CTNNBL1 and $P D E 7 A$ the minor alleles were associated with a reduced rate of cognitive decline. At baseline, $B D N F$ and $P D E 7 A$ minor alleles were associated with worse cognitive performance, while SORL1 and ZNF224 minor alleles were associated with better cognitive performance. FRMD4A-rs7081208 was associated with a reduced and a greater rate of decline for working memory and vocabulary respectively, though at baseline working memory was associated with worse performance while vocabulary was associated with better performance. Additionally, FRMD 4A-rs17314229 minor allele were associated with a greater rate of decline in Working memory.

In comparison to the top $\mathrm{AD}$ related SNPs, the additional $\mathrm{AD}$ related genetic variants in FRMD4A, SORL1 and ZNF224 were associated with cognitive performance in vocabulary and working memory, potentially indicating that they may be involved in the development of atypical $\mathrm{AD}$, in which the development of non-amnestic cognitive deficits occurs early on in the disease process (Dubois et al., 2010). For the cognition related genetic variants, SNPs in COMT and CTNNBL1 have been associated with differences in regional brain structures and activations that are involved in episodic memory processes, potentially explaining the differential associations of these variants with episodic memory (Papassotiropoulos et al., 2013; Witte and Flöel, 2012). BDNF is widely expressed in the prefrontal cortex, which is associated, amongst other functions, with working memory (Galloway et al., 2008). As with the LOAD risk loci, the additional SNPs were primarily associated with cognitive decline in participants who were classified cognitively impaired. However, as with the LOAD 
risk loci, the effect sizes for these SNPs were small and inclusion of the SNPs in the model resulted in a negligible increase in the amount of explained variability in cognitive performance.

The presented findings should be interpreted in conjunction with some study limitations. The sample used in this study is somewhat better educated than the population from which it was drawn. Higher education is associated with a reduced risk of cognitive decline and incident dementia. Additionally, the sample is relatively young, which in combination with a higher level of education could limit our ability to detect an effect of the genetic factor with cognitive decline. This is possibly reflected in the limited person specific variation in the rate of decline in the linear mixed models. Second, the subjects in this study are Caucasian and as such our findings need to be replicated in other ethnic groups. Third, despite excluding individuals with probable dementia at each wave, it is still possible that individuals in the preclinical phase of dementia were included in the analysis. Fourth, in concordance with the avaliable data, we have specified time as linear, however, cognitive decline may accelerate at older ages (Wilson et al., 2009) highlighting the need to investigate nonlinear cognitive trajectories (Weuve et al., 2015). Finally, although we have a strong a priori evidence for all our hypothesis, it should be noted that correcting for multiple testing using Bonferroni correction, all corrected $p$ values would have yielded non-significant results.

Despite these limitations however, this study investigated a large community based cohort followed longitudinally for a period of eight years, with three waves of assessment that included a comprehensive cognitive assessment of different cognitive abilities. These strengths allow for a robust statistical inference about the effect the selected genetic factors have on non-clinical cognitive decline. The narrow age-cohort design also reduced the impact of age-differences influencing results. 
434

435

436

437

438

439

440

441

442

443

444

445

446

447

448

449

450

451

452

453

454

To conclude, our findings suggest that the majority of LOAD risk genes are not individually associated with non-clinical cognitive decline in a cohort of older adults who were followed for a period of 8 years. When considered collectively as a genetic risk score, the observed associations are due to the significantly larger weight associated with $A P O E{ }^{*} \epsilon 4$ allele. The PATH study is ongoing and the number of incident cases of mild cognitive impairment and dementia among participants is increasing. The work presented here thus provides an excellent basis for further investigating the effects of $\mathrm{AD}$ risk variants in non-pathological versus pathological decline (Knight et al., 2014), gene-gene interactions (Barral et al., 2012; Engelman et al., 2013) and gene-environment interactions (Ferencz et al., 2014) in future studies.

\section{Acknowledgements}

We thank the participants of the PATH study, Peter Butterworth, Andrew Mackinnon, Anthony Jorm, Bryan Rodgers, Helen Christensen, Patricia Jacomb, Karen Mawell and Jorge Velez. The study was supported by the Dementia Collaborative Research Centers and the National Health and Medical Research Council (NHMRC) grants 973302, 179805, 1002160. DD is funded by NHMRC Project Grant No. 1043256. NC is funded by Research Fellowship No. 12010227. KJA is funded by NHMRC Research Fellowship No. 1102694.

\section{Disclosures}

The authors have no conflict of interests to report. 


\section{${ }_{455}$ References}

Anstey, K.J., Christensen, H., Butterworth, P., Easteal, S., Mackinnon, A., Jacomb, T., Maxwell, K., Rodgers, B., Windsor, T., Cherbuin, N., Jorm, A.F., 2012. Cohort profile: the PATH through life project. International Journal of Epidemiology 41, 951-960.

Baddeley, A., Emslie, H., Nimmo-Smith, I., 1993. The Spot-the-Word test: a robust estimate of verbal intelligence based on lexical decision. The British journal of Clinical Psychology 32, 55-65.

Barral, S., Bird, T., Goate, A., Farlow, M.R., Diaz-Arrastia, R., Bennett, D.A., Graff-Radford, N., Boeve, B.F., Sweet, R.A., Stern, Y., Wilson, R.S., Foroud, T., Ott, J., Mayeux, R., 2012. Genotype patterns at PICALM, CR1, BIN1, CLU, and APOE genes are associated with episodic memory. Neurology 78, 1464-1471.

Barton, K., Barton, M.K., 2013. Package 'MuMIn'. Version .

Bates, D., Maechler, M., Bolker, B., Walker, S., 2014. lme4: Linear mixed-effects models using Eigen and S4. URL: http://CRAN.R-project.org/package=lme4. r package version 1.1-7.

Bertram, L., McQueen, M.B., Mullin, K., Blacker, D., Tanzi, R.E., $2007 . \quad$ Systematic meta-analyses of Alzheimer disease genetic association studies: the AlzGene database. Nature Genetics 39, 17-23.

Boyle, P.A., Wilson, R.S., Yu, L., Barr, A.M., Honer, W.G., Schneider, J.A., Bennett, D.A., 2013a. Much of late life cognitive decline is not due to common neurodegenerative pathologies. Annals of Neurology 74, 478-489.

Boyle, P.A., Yu, L., Wilson, R.S., Gamble, K., Buchman, A.S., Bennett, D.A., 2012. Poor decision making is a consequence of cognitive decline among older persons without Alzheimer's disease or mild cognitive impairment. PLoS ONE 7, e43647. 
Boyle, P.A., Yu, L., Wilson, R.S., Schneider, J.A., Bennett, D.A., 2013b. Relation of neuropathology with cognitive decline among older persons without dementia. Frontiers in Aging Neuroscience 5, 50 .

Carrasquillo, M.M., Crook, J.E., Pedraza, O., Thomas, C.S., Pankratz, V.S., Allen, M., Nguyen, T., Malphrus, K.G., Ma, L., Bisceglio, G.D., Roberts, R.O., Lucas, J.A., Smith, G.E., Ivnik, R.J., Machulda, M.M., Graff-Radford, N.R., Petersen, R.C., Younkin, S.G., Ertekin-Taner, N., 2015. Late-onset Alzheimer's risk variants in memory decline, incident mild cognitive impairment, and Alzheimer's disease. Neurobiology of Aging 36, 60-67.

Che, R., Motsinger-Reif, A.A., 2013. Evaluation of genetic risk score models in the presence of interaction and linkage disequilibrium. Frontiers in Genetics 4, 138.

Davies, G., Armstrong, N., Bis, J.C., Bressler, J., Chouraki, V., Giddaluru, S., Hofer, E., Ibrahim-Verbaas, C.A., Kirin, M., Lahti, J., van der Lee, S.J., Le Hellard, S., Liu, T., Marioni, R.E., Oldmeadow, C., Postmus, I., Smith, A.V., Smith, J.A., Thalamuthu, A., Thomson, R., Vitart, V., Wang, J., Yu, L., Zgaga, L., Zhao, W., Boxall, R., Harris, S.E., Hill, W.D., Liewald, D.C., Luciano, M., Adams, H., Ames, D., Amin, N., Amouyel, P., Assareh, A.A., Au, R., Becker, J.T., Beiser, A., Berr, C., Bertram, L., Boerwinkle, E., Buckley, B.M., Campbell, H., Corley, J., De Jager, P.L., Dufouil, C., Eriksson, J.G., Espeseth, T., Faul, J.D., Ford, I., Scotland, G., Gottesman, R.F., Griswold, M.E., Gudnason, V., Harris, T.B., Heiss, G., Hofman, A., Holliday, E.G., Huffman, J., Kardia, S.L.R., Kochan, N., Knopman, D.S., Kwok, J.B., Lambert, J.C., Lee, T., Li, G., Li, S.C., Loitfelder, M., Lopez, O.L., Lundervold, A.J., Lundqvist, A., Mather, K.A., Mirza, S.S., Nyberg, L., Oostra, B.A., Palotie, A., Papenberg, G., Pattie, A., Petrovic, K., Polasek, O., Psaty, B.M., Redmond, P., Reppermund, S., Rotter, J.I., Schmidt, H., Schuur, M., Schofield, P.W., Scott, R.J., Steen, V.M., Stott, D.J., van Swieten, J.C., Taylor, K.D., Trollor, J., Trompet, S., Uitterlinden, A.G., Weinstein, G., Widen, E., Windham, B.G., Jukema, J.W., Wright, A.F., Wright, M.J., Yang, Q., Amieva, H., 
Attia, J.R., Bennett, D.A., Brodaty, H., de Craen, A.J.M., Hayward, C., Ikram, M.A., Lindenberger, U., Nilsson, L.G., Porteous, D.J., Raikkonen, K., Reinvang, I., Rudan, I., Sachdev, P.S., Schmidt, R., Schofield, P.R., Srikanth, V., Starr, J.M., Turner, S.T., Weir, D.R., Wilson, J.F., van Duijn, C., Launer, L., Fitzpatrick, A.L., Seshadri, S., Mosley, T.H.J., Deary, I.J., 2015. Genetic contributions to variation in general cognitive function: a meta-analysis of genome-wide association studies in the CHARGE consortium ( $=53$ 949). Molecular Psychiatry 20, 183-192.

Davies, G., Harris, S.E., Reynolds, C.A., Payton, A., Knight, H.M., Liewald, D.C., Lopez, L.M., Luciano, M., Gow, A.J., Corley, J., Henderson, R., Murray, C., Pattie, A., Fox, H.C., Redmond, P., Lutz, M.W., Chiba-Falek, O., Linnertz, C., Saith, S., Haggarty, P., McNeill, G., Ke, X., Ollier, W., Horan, M., Roses, A.D., Ponting, C.P., Porteous, D.J., Tenesa, A., Pickles, A., Starr, J.M., Whalley, L.J., Pedersen, N.L., Pendleton, N., Visscher, P.M., Deary, I.J., 2014. A genome-wide association study implicates the APOE locus in nonpathological cognitive ageing. Molecular Psychiatry 19, 76-87.

Davies, G., Tenesa, A., Payton, A., Yang, J., Harris, S.E., Liewald, D., Ke, X., Le Hellard, S., Christoforou, A., Luciano, M., McGhee, K., Lopez, L., Gow, A.J., Corley, J., Redmond, P., Fox, H.C., Haggarty, P., Whalley, L.J., McNeill, G., Goddard, M.E., Espeseth, T., Lundervold, A.J., Reinvang, I., Pickles, A., Steen, V.M., Ollier, W., Porteous, D.J., Horan, M., Starr, J.M., Pendleton, N., Visscher, P.M., Deary, I.J., 2011. Genome-wide association studies establish that human intelligence is highly heritable and polygenic. Molecular Psychiatry 16, 996-1005.

De Jager, P.L., Shulman, J.M., Chibnik, L.B., Keenan, B.T., Raj, T., Wilson, R.S., Yu, L., Leurgans, S.E., Tran, D., Aubin, C., Anderson, C.D., Biffi, A., Corneveaux, J.J., Huentelman, M.J., Rosand, J., Daly, M.J., Myers, A.J., Reiman, E.M., Bennett, D.A., Evans, D.A., 2012. A genome-wide scan for common variants affecting the rate of age-related cognitive decline. Neurobiology of Aging 33, 1017.e1-1017.e15. 
Deary, I.J., Yang, J., Davies, G., Harris, S.E., Tenesa, A., Liewald, D., Luciano, M., Lopez, L.M., Gow, A.J., Corley, J., Redmond, P., Fox, H.C., Rowe, S.J., Haggarty, P., McNeill, G., Goddard, M.E., Porteous, D.J., Whalley, L.J., Starr, J.M., Visscher, P.M., 2012. Genetic contributions to stability and change in intelligence from childhood to old age. Nature 482, 212-215.

Delis, D.C., Kramer, J.H., Kaplan, E., Ober, B.A., 1987. California Verbal Learning Test. Psychological Corporation, San Antonio.

Dubois, B., Feldman, H.H., Jacova, C., Cummings, J.L., DeKosky, S.T., Barberger-Gateau, P., Delacourte, A., Frisoni, G., Fox, N.C., Galasko, D., Gauthier, S., Hampel, H., Jicha, G.A., Meguro, K., O’Brien, J., Pasquier, F., Robert, P., Rossor, M., Salloway, S., Sarazin, M., de Souza, L.C., Stern, Y., Visser, P.J., Scheltens, P., 2010. Revising the definition of Alzheimer's disease: a new lexicon. The Lancet Neurology 9, 1118-1127.

Engelman, C.D., Koscik, R.L., Jonaitis, E.M., Okonkwo, O.C., Hermann, B.P., La Rue, A., Sager, M.A., 2013. Interaction between two cholesterol metabolism genes influences memory: findings from the Wisconsin Registry for Alzheimer's Prevention. Journal of Alzheimer's Disease 36, 749-757.

Farrer, L.A., Cupples, L.A., Haines, J.L., Hyman, B., Kukull, W.A., Mayeux, R., Myers, R.H., Pericak-Vance, M.A., Risch, N., van Duijn, C.M., 1997. Effects of Age, Sex, and Ethnicity on the Association Between Apolipoprotein E Genotype and Alzheimer Disease: A Meta-analysis. JAMA 278, 1349-1356.

Ferencz, B., Jonsson Laukka, E., Welmer, A.K., Kalpouzos, G., Angleman, S., Keller, L., Graff, C., Lovden, M., Backman, L., 2014. The Benefits of Staying Active in Old Age: Physical Activity Counteracts the Negative Influence of PICALM, BIN1, and CLU Risk Alleles on Episodic Memory Functioning. Psychology and Aging 52, 181-184.

Folstein, M.F., Folstein, S.E., McHugh, P.R., 1975. "Mini-mental state". A practical 
method for grading the cognitive state of patients for the clinician. Journal of Psychiatric Research 12, 189-198.

Galloway, E.M., Woo, N.H., Lu, B., 2008. Chapter 15 Persistent neural activity in the prefrontal cortex: A mechanism by which BDNF regulates working memory?, in: Castellucci, W.S.S.V.F., Sylvie Belleville, J.C.L. (Eds.), Essence of Memory. Elsevier, pp. 251-266.

Halekoh, U., Højsgaard, S., 2012. A Kenward-Roger approximation and parametric bootstrap methods for tests in linear mixed models-the R package pbkrtest. Journal of Statistical Software 59, 17-23.

Harris, S.E., Davies, G., Luciano, M., Payton, A., Fox, H.C., Haggarty, P., Ollier, W., Horan, M., Porteous, D.J., Starr, J.M., Whalley, L.J., Pendleton, N., Deary, I.J., 2014. Polygenic Risk for Alzheimer's Disease is not Associated with Cognitive Ability or Cognitive Aging in Non-Demented Older People. Journal of Alzheimer's Disease 39, $565-574$.

Harris, S.E., Deary, I.J., 2011. The genetics of cognitive ability and cognitive ageing in healthy older people. Trends in Cognitive Sciences 15, 388-394.

Hintze, J.L., Nelson, R.D., 1998. Violin plots: a box plot-density trace synergism. The American Statistician 29, 440-449.

Johnson, P., 2014. Extension of Nakagawa \& Schielzeth's R2GLMM to random slopes models. Methods in Ecology and Evolution 5, 944-946.

Jorm, A.F., Mather, K.A., Butterworth, P., Anstey, K.J., Christensen, H., Easteal, S., 2007. APOE genotype and cognitive functioning in a large age-stratified population sample. Neuropsychology 21, 1-8.

Keller, J., 2006. Age-related neuropathology, cognitive decline, and Alzheimer's disease. Ageing Research Reviews 5, 1-13. 
Knight, R.G., Tsui, H.S.L., Abraham, W.C., Skeaff, C.M., McMahon, J.A., Cutfield, N.J., 2014. Lack of effect of the apolipoprotein E epsilon4 genotype on cognition during healthy aging. Journal of Clinical and Experimental Neuropsychology 36, 1-9.

Kobayashi, L.C., Wardle, J., Wolf, M.S., von Wagner, C., 2014. Aging and Functional Health Literacy: A Systematic Review and Meta-Analysis. The Journals of Gerontology Series B: Psychological Sciences and Social Sciences gbu161.

McFall, G.P., Wiebe, S.A., Vergote, D., Anstey, K.J., Dixon, R.A., 2015. Alzheimer's genetic risk intensifies neurocognitive slowing associated with diabetes in nondemented older adults. Alzheimer's \& Dementia: Diagnosis, Assessment \& Disease Monitoring 1, 395-402.

Mengel-From, J., Thinggaard, M., Lindahl-Jacobsen, R., McGue, M., Christensen, K., Christiansen, L., 2013. CLU genetic variants and cognitive decline among elderly and oldest old. PLoS ONE 8, e79105.

Morgan, K., Carrasquillo, M.M., 2013. Genetic Variants in Alzheimer's Disease. Springer Science \& Business Media.

Nakagawa, S., Schielzeth, H., 2013. A general and simple method for obtaining R2 from generalized linear mixed-effects models. Methods in Ecology and Evolution 4, 133-142.

Papassotiropoulos, A., Stefanova, E., Vogler, C., Gschwind, L., Ackermann, S., Spalek, K., Rasch, B., Heck, A., Aerni, A., Hanser, E., Demougin, P., Huynh, K.D., Luechinger, R., Klarhofer, M., Novakovic, I., Kostic, V., Boesiger, P., Scheffler, K., de Quervain, D.J.F., 2013. A genome-wide survey and functional brain imaging study identify CTNNBL1 as a memory-related gene. Molecular Psychiatry 18, 255-263.

Payton, A., 2009. The Impact of Genetic Research on our Understanding of Normal Cognitive Ageing: 1995 to 2009. Neuropsychology Review 19, 451-477. 
Purcell, S., Neale, B., Todd-Brown, K., Thomas, L., Ferreira, M.A.R., Bender, D., Maller, J., Sklar, P., de Bakker, P.I.W., Daly, M.J., Sham, P.C., 2007. PLINK: a tool set for whole-genome association and population-based linkage analyses. American journal of human genetics 81, 559-575.

R Core Team, 2013. R: A Language and Environment for Statistical Computing. R Foundation for Statistical Computing. Vienna, Austria. URL: http://www.R-project.org/. ISBN 3-900051-07-0.

Savva, G.M., Wharton, S.B., Ince, P.G., Forster, G., Matthews, F.E., Brayne, C., 2009. Age, Neuropathology, and Dementia. New England Journal of Medicine 360, 2302-2309.

Smith, A., 2002. Symbol digit modalities test: Manual. Western Psychological Corporation, Los Angeles.

Stekhoven, D.J., Bühlmann, P., 2012. MissForest-non-parametric missing value imputation for mixed-type data. Bioinformatics 28, 112-118.

Thambisetty, M., Beason-Held, L.L., An, Y., Kraut, M., Nalls, M., Hernandez, D.G., Singleton, A.B., ZONDERMAN, A.B., Ferrucci, L., Lovestone, S., RESNICK, S.M., 2013. Alzheimer Risk Variant CLU and Brain Function During Aging. Biological psychiatry 73, 399-405.

Tucker-Drob, E.M., 2011. Neurocognitive functions and everyday functions change together in old age. Neuropsychology 25, 368-377.

Vivot, A., Glymour, M.M., Tzourio, C., Amouyel, P., Chene, G., Dufouil, C., 2015. Association of Alzheimer's related genotypes with cognitive decline in multiple domains: results from the Three-City Dijon study. Molecular Psychiatry 20, 1173-1178.

Wechsler, D., 1945. A standardized memory scale for clinical use. The Journal of Psychology 19, 87-95. 
Weuve, J., Proust-Lima, C., Power, M.C., Gross, A.L., Hofer, S.M., Thiébaut, R., Chêne, G., Glymour, M.M., Dufouil, C., 2015. Guidelines for reporting methodological challenges and evaluating potential bias in dementia research. Alzheimer's \& Dementia 11, 1098-1109.

Wilson, R.S., Beckett, L.A., Barnes, L.L., Schneider, J.A., Bach, J., Evans, D.A., Bennett, D.A., 2002. Individual differences in rates of change in cognitive abilities of older persons. Psychology and Aging 17, 179-193.

Wilson, R.S., Boyle, P.A., Segawa, E., Yu, L., Begeny, C.T., Anagnos, S.E., Bennett, D.A., 2013. The influence of cognitive decline on well-being in old age. Psychology and Aging $28,304-313$.

Wilson, R.S., Hebert, L.E., Scherr, P.A., Barnes, L.L., Mendes de Leon, C.F., Evans, D.A., 2009. Educational attainment and cognitive decline in old age. Neurology 72, 460-465.

Wisdom, N.M., Callahan, J.L., Hawkins, K.A., 2011. The effects of apolipoprotein E on non-impaired cognitive functioning: A meta-analysis. Neurobiology of Aging 32, 63-74.

Witte, A.V., Flöel, A., 2012. Effects of COMT polymorphisms on brain function and behavior in health and disease. Brain Research Bulletin 88, 418-428.

Yam, A., Marsiske, M., 2013. Cognitive longitudinal predictors of older adults' self-reported IADL function. Journal of Aging and Health 25, 163S-85S.

Zahodne, L.B., Manly, J.J., MacKay-Brandt, A., Stern, Y., 2013. Cognitive declines precede and predict functional declines in aging and Alzheimer's disease. PLoS ONE 8, e73645.

Zhang, C., Pierce, B.L., 2014. Genetic susceptibility to accelerated cognitive decline in the US Health and Retirement Study. Neurobiology of Aging 35, 1512.e11-8. 
Table 1: Sample Demographics

\begin{tabular}{lccccc}
\hline & $\begin{array}{c}\text { Excluded } \\
(n=861)\end{array}$ & $\begin{array}{c}\text { Included } \\
(n=1,689)\end{array}$ & $\begin{array}{c}\text { Degrees of } \\
\text { Freedom }\end{array}$ & $t / \chi^{2}$ & $p$ \\
\hline Age $^{\dagger}$ & $62.46 \pm 1.49$ & $62.54 \pm 1.51$ & 1,753 & -1.22 & 0.21 \\
Education $^{\dagger}$ & $3.31 \pm 3.09$ & $14 \pm 2.59$ & 1,488 & -5.62 & $<0.001$ \\
MMSE $^{\dagger}$ & $28.6 \pm 2.13$ & $29.35 \pm 0.92$ & 1010 & -9.77 & $<0.001$ \\
Male $n(\%)^{\ddagger}$ & $443(51.4 \%)$ & $873(51.7 \%)$ & 1 & 0.005 & 0.94 \\
APOE Genotypes $n(\%)$ & & & & \\
${ }^{*} \epsilon 2 /{ }^{*} \epsilon 2$ & $6(0.70 \%)$ & $13(0.77 \%)$ & & & \\
${ }^{*} \epsilon 3 /{ }^{*} \epsilon 3$ & $395(45.82 \%)$ & $1048(62 \%)$ & & & \\
${ }^{*} \epsilon 4 /{ }^{*} \epsilon 4$ & $20(2.32 \%)$ & $29(1.71 \%)$ & & & \\
${ }^{*} \epsilon 2 /{ }^{*} \epsilon 3$ & $70(8.12 \%)$ & $204(12.07 \%)$ & & & \\
$*_{\epsilon 2}{ }^{*} \epsilon 4$ & $60(6.96 \%)$ & $0(0 \%)$ & & & \\
${ }^{*} \epsilon 3 /{ }^{*} \epsilon 4$ & $137(15.89 \%)$ & $395(23.37 \%)$ & & & \\
\hline
\end{tabular}

${ }^{\dagger}$ Unpaired 2-tailed t-test. ${ }^{\ddagger}$ Pearson’s $\chi^{2}$ 2-tailed test. 

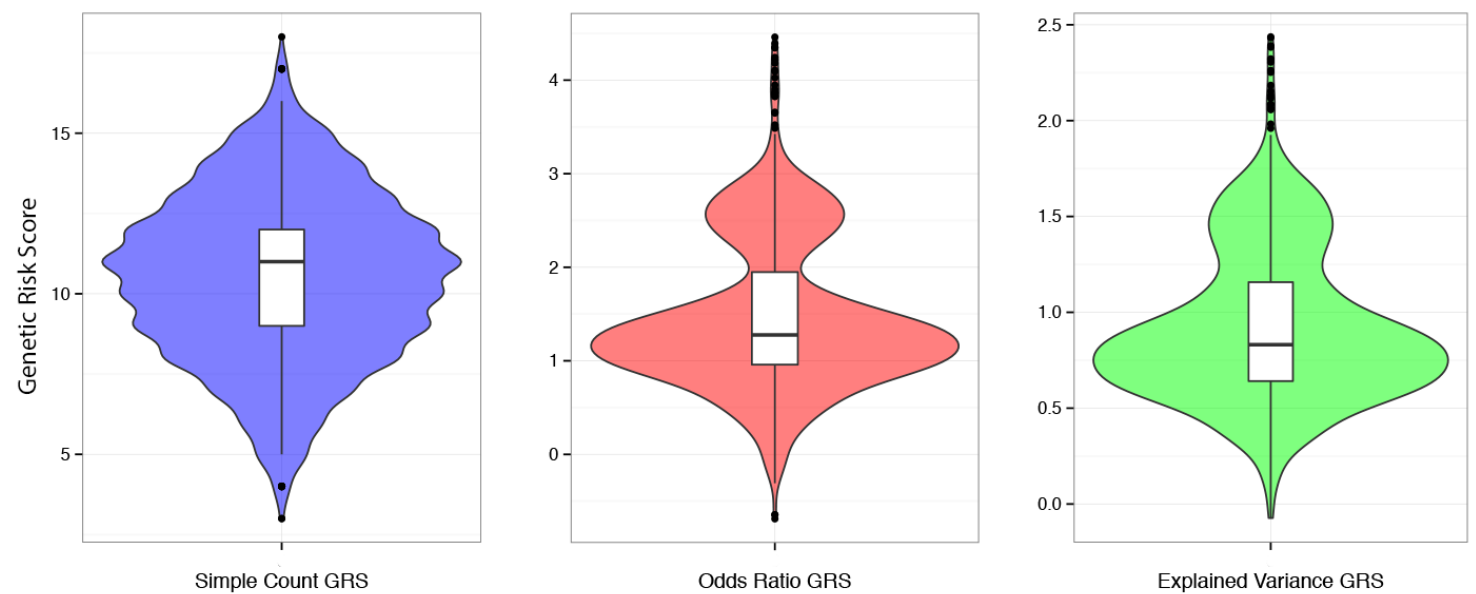

Figure 1: Distributions of the three genetic risk scores: SC-GRS $($ Mean $=10.5 ;$ sd $=2.58)$, OR-GRS $($ Mean $=1.47 ; \mathrm{sd}=0.8)$ and EV-GRS $($ Mean $=0.92 ; \mathrm{sd}=0.4)$. The variable widths of each violin plot indicate the probability density of the data at each score, with the box plots indicating the first, median and third quartile (Hintze and Nelson, 1998). 
Table 2: Top LOAD risk SNPs and GRS: Parameter estimates and model fit statistics for SNP/GRS main effects

\begin{tabular}{|c|c|c|c|c|c|}
\hline & & Episodic & Digits & Spot-the-Word & Symbol Digits \\
\hline & & Memory & Backwards & & Modalities Test \\
\hline & & Estimate (SE) & Estimate (SE) & Estimate (SE) & Estimate (SE) \\
\hline \multirow[t]{2}{*}{$A P O E \in \mathcal{2}$} & Intercept & $-0.02(0.07)$ & $0.01(0.07)$ & $0.06(0.06)$ & $-0.04(0.06)$ \\
\hline & Slope & $-0.00(0.01)$ & $0.01(0.01)$ & $-0.00(0.00)$ & $0.01(0.01)$ \\
\hline \multirow[t]{3}{*}{$A P O E \in 4$} & Intercept & $0.03(0.05)$ & $0.02(0.05)$ & $0.02(0.05)$ & $-0.01(0.05)$ \\
\hline & Slope & $-0.02(0.01)^{* *}$ & $0.01(0.01)$ & $-0.00(0.00)$ & $-0.01(0.00)$ \\
\hline & $F$-test & $2.61^{*}$ & 1.78 & 0.30 & 0.80 \\
\hline \multirow[t]{3}{*}{$A B C A$ 7-rs3764650 } & Intercept & $-0.12(0.06)^{*}$ & $0.02(0.06)$ & $-0.05(0.05)$ & $-0.04(0.06)$ \\
\hline & Slope & $-0.0003(0.007)$ & $0.004(0.007)$ & $-0.0002(0.004)$ & $0.01(0.01)$ \\
\hline & $F$-test & 2.93 & 0.46 & 0.59 & 0.84 \\
\hline \multirow[t]{3}{*}{ BIN1-rs744373 } & Intercept & $-0.03(0.04)$ & $0.07(0.04)$ & $-0.00(0.04)$ & $0.04(0.04)$ \\
\hline & Slope & $-0.006(0.005)$ & $0.0002(0.005)$ & $-0.002(0.003)$ & $-0.001(0.004)$ \\
\hline & $F$-test & 1.71 & 1.78 & 0.18 & 0.49 \\
\hline \multirow[t]{3}{*}{ CD2AP-rs9296559 } & Intercept & $0.02(0.04)$ & $0.03(0.05)$ & $-0.02(0.04)$ & $0.004(0.04)$ \\
\hline & Slope & $-0.006(0.006)$ & $-0.001(0.005)$ & $-0.003(0.003)$ & $-0.001(0.004)$ \\
\hline & $F$-test & 0.56 & 0.27 & 0.91 & 0.03 \\
\hline CD33-rs34813869 & Intercept & $-0.02(0.07)$ & $-0.05(0.07)$ & $-0.07(0.06)$ & $0.06(0.07)$ \\
\hline
\end{tabular}




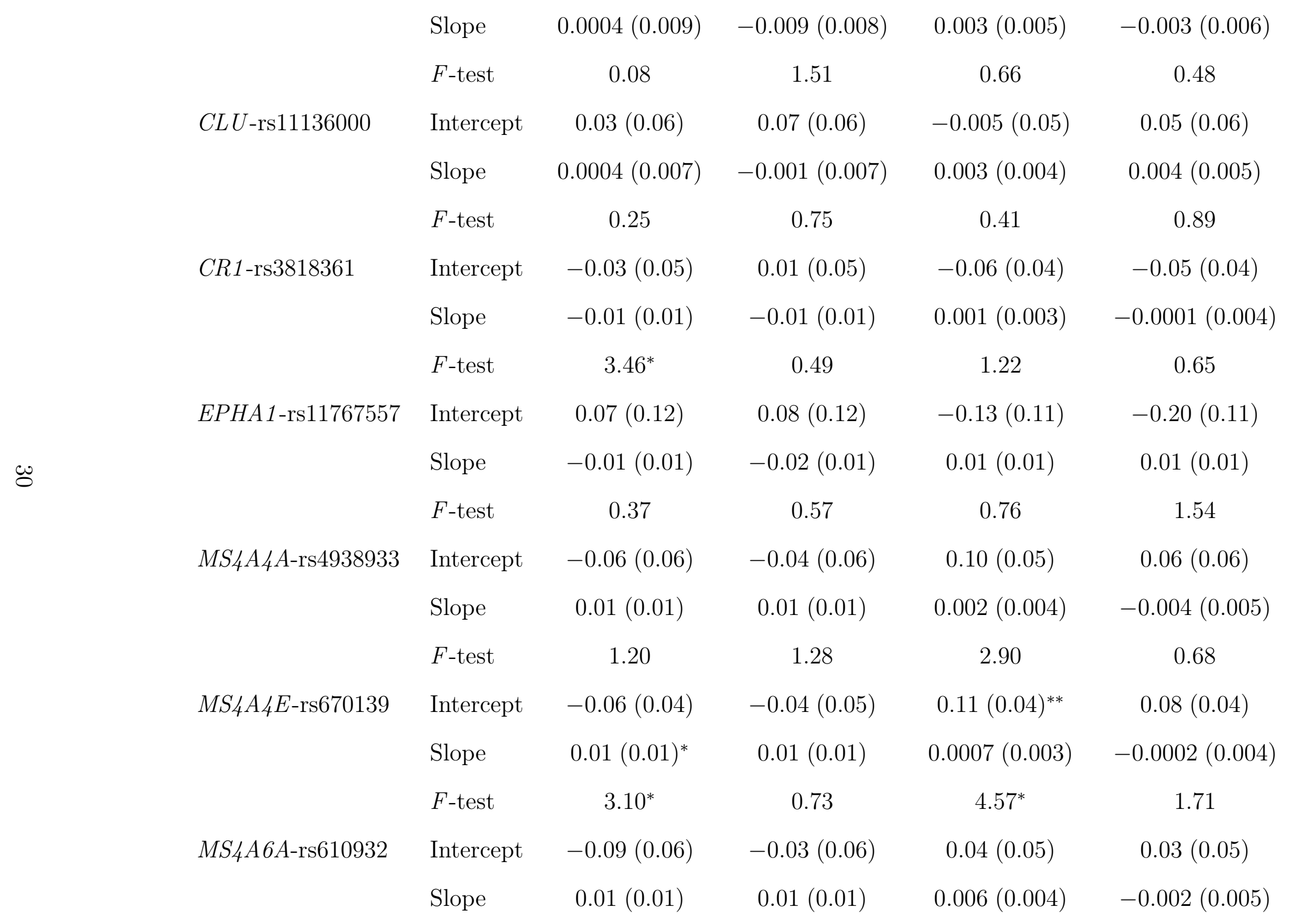




\begin{tabular}{llcccc} 
& $F$-test & 1.64 & 1.39 & 2.12 & 0.17 \\
PICALM-rs3851179 & Intercept & $0.01(0.06)$ & $-0.03(0.06)$ & $-0.02(0.06)$ & $0.01(0.06)$ \\
& Slope & $0.0001(0.008)$ & $0.006(0.007)$ & $0.003(0.004)$ & $0.009(0.006)$ \\
& $F$-test & 0.01 & 0.35 & 0.19 & 1.41 \\
SC-GRS & Intercept & $-0.01(0.009)$ & $-0.001(0.009)$ & $0.001(0.008)$ & $0.008(0.008)$ \\
& Slope & $-0.001(0.001)$ & $0.0006(0.001)$ & $-0.0005(0.0006)$ & $0.0002(0.0008)$ \\
& F-test & 1.95 & 0.18 & 0.35 & 0.53 \\
OR-GRS & Intercept & $-0.00001(0.03)$ & $-0.003(0.03)$ & $-0.001(0.02)$ & $0.007(0.03)$ \\
& Slope & $-0.009(0.003)^{*}$ & $0.002(0.003)$ & $-0.002(0.002)$ & $-0.004(0.003)$ \\
& F-test & $4.20^{*}$ & 0.13 & 0.58 & 1.01 \\
& Intercept & $-0.007(0.05)$ & $-0.007(0.06)$ & $0.004(0.05)$ & $0.02(0.05)$ \\
& Slope & $-0.017(0.007)^{*}$ & $0.004(0.007)$ & $-0.004(0.004)$ & $-0.006(0.005)$ \\
& F-GRS & $4.44^{*}$ & 0.20 & 0.64 & 0.75 \\
\hline
\end{tabular}


Table 3: Additional SNPs: Model Fit for SNP main effects

\begin{tabular}{|c|c|c|c|c|c|}
\hline & & Episodic & Digits & Spot-the-Word & Symbol Digits \\
\hline & & Memeory & Backwards & & Modalities Test \\
\hline & & Estimate (SE) & Estimate (SE) & Estimate (SE) & Estimate (SE) \\
\hline \multirow[t]{3}{*}{$B D N F$-rs 6265} & Intercept & $0.002(0.05)$ & $-0.1(0.05)^{*}$ & $-0.02(0.04)$ & $0.05(0.05)$ \\
\hline & Slope & $-0.008(0.006)$ & $-0.003(0.006)$ & $-0.005(0.003)$ & $0.001(0.004)$ \\
\hline & $F$-test & 1.31 & $3.25^{*}$ & 1.75 & 0.64 \\
\hline \multirow[t]{3}{*}{$C E T P$-rs5882 } & Intercept & $0.005(0.043)$ & $-0.053(0.045)$ & $-0.007(0.039)$ & $-0.003(0.042)$ \\
\hline & Slope & $-0.0002(0.005)$ & $0.01(0.005)$ & $-0.005(0.003)$ & $-0.002(0.004)$ \\
\hline & $F$-test & 0.01 & 1.77 & 1.77 & 0.13 \\
\hline \multirow[t]{3}{*}{$C O M T-\mathrm{rs} 4680$} & Intercept & $0.078(0.050)$ & $0.032(0.052)$ & $0.033(0.045)$ & $-0.005(0.049)$ \\
\hline & Slope & $-0.015(0.006)^{*}$ & $-0.003(0.006)$ & $0.004(0.004)$ & $-0.009(0.005)$ \\
\hline & $F$-test & $3.09^{*}$ & 0.22 & 1.57 & 2.09 \\
\hline \multirow[t]{3}{*}{$C T N N B L 1$-rs6125962 } & Intercept & $-0.054(0.067)$ & $-0.017(0.07)$ & $0.024(0.061)$ & $-0.077(0.065)$ \\
\hline & Slope & $0.018(0.008)^{*}$ & $0.010(0.008)$ & $-0.002(0.005)$ & $0.006(0.006)$ \\
\hline & $F$-test & 2.27 & 0.89 & 0.13 & 0.90 \\
\hline \multirow[t]{3}{*}{ FRMD4A-rs17314229 } & Intercept & $-0.022(0.043)$ & $0.04(0.045)$ & $0.037(0.039)$ & $-0.006(0.042)$ \\
\hline & Slope & $0.007(0.005)$ & $-0.010(0.005)^{*}$ & $-0.005(0.003)$ & $0.001(0.004)$ \\
\hline & $F$-test & 0.75 & 1.96 & 1.33 & 0.04 \\
\hline
\end{tabular}




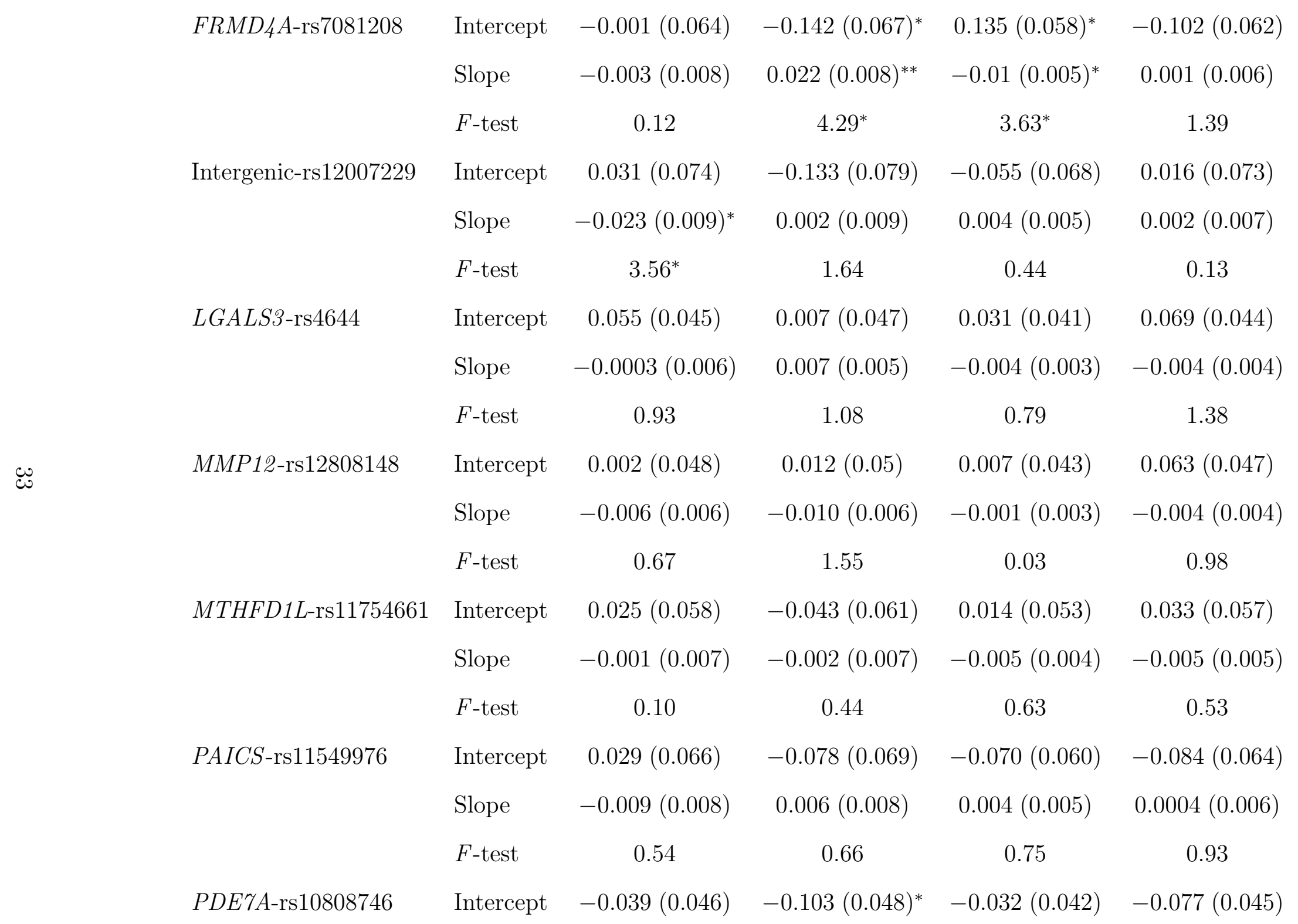




\begin{tabular}{|c|c|c|c|c|c|}
\hline & Slope & $0.006(0.006)$ & $0.010(0.006)$ & $-0.000(0.003)$ & $0.009(0.004)^{*}$ \\
\hline & $F$-test & 0.69 & 2.60 & 0.35 & 2.76 \\
\hline SNTG1-rs16914781 & Intercept & $0.086(0.046)$ & $0.038(0.048)$ & $-0.048(0.042)$ & $0.008(0.045)$ \\
\hline & Slope & $-0.008(0.006)$ & $-0.002(0.006)$ & $0.0002(0.003)$ & $-0.001(0.004)$ \\
\hline & $F$-test & 1.94 & 0.31 & 0.74 & 0.06 \\
\hline SORL1-rs668387 & Intercept & $0.046(0.047)$ & $0.006(0.049)$ & $0.084(0.043)^{*}$ & $0.010(0.046)$ \\
\hline & Slope & $-0.007(0.006)$ & $0.007(0.006)$ & $-0.006(0.003)$ & $0.005(0.004)$ \\
\hline & $F$-test & 0.85 & 0.94 & 2.73 & 0.92 \\
\hline SPON1-rs11023139 & Intercept & $0.058(0.069)$ & $-0.093(0.072)$ & $-0.104(0.063)$ & $0.009(0.067)$ \\
\hline U & Slope & $-0.009(0.009)$ & $0.007(0.008)$ & $0.007(0.005)$ & $-0.004(0.006)$ \\
\hline & $F$-test & 0.62 & 0.88 & 1.69 & 0.17 \\
\hline ZNF224-rs3746319 & Intercept & $0.012(0.046)$ & $0.071(0.049)$ & $0.100(0.042)^{*}$ & $0.047(0.045)$ \\
\hline & Slope & $-0.002(0.006)$ & $0.007(0.006)$ & $-0.001(0.003)$ & $0.005(0.004)$ \\
\hline & $F$-test & 0.06 & $3.43^{*}$ & $3.04^{*}$ & 1.81 \\
\hline
\end{tabular}


Supplementary Data 
Table 1: SNPs used in this study

\begin{tabular}{|c|c|c|c|c|c|c|}
\hline Gene & Protein & SNP & Chromosome & Alleles ${ }^{\dagger}$ & $\mathrm{MAF}^{\ddagger}$ & Odds Ratio ${ }^{\S}$ \\
\hline \multicolumn{7}{|c|}{ Top Report Alzheimer's disease risk SNPs } \\
\hline$A P O E$ & Apolipoprotein E & rs429358/rs7412 & 19 & $\epsilon 2 / \epsilon 3 / \epsilon 4$ & $0.8 / 0.14$ & $0.54 / 3.81$ \\
\hline$A B C A Y$ & $\begin{array}{l}\text { ATP-binding cassette subfamily A } \\
\text { member } 7\end{array}$ & rs3764650 & 19 & $\mathrm{~T} / \mathrm{G}$ & 0.11 & 1.23 \\
\hline$B I N 1$ & Myc box-dependent-interacting protein 1 & rs744373 & 2 & $\mathrm{~A} / \mathrm{G}$ & 0.31 & 1.17 \\
\hline$C D \mathscr{2 A P}$ & CD2-associated protein & rs9296559 & 6 & $\mathrm{~T} / \mathrm{C}$ & 0.27 & 1.11 \\
\hline CDЗ3 & Myeloid cell surface antigen CD33 & rs34813869 & 19 & $\mathrm{~A} / \mathrm{G}$ & 0.29 & 0.89 \\
\hline$C L U$ & Clusterin & rs11136000 & 8 & $\mathrm{C} / \mathrm{T}$ & 0.35 & 0.88 \\
\hline$C R 1$ & Complement receptor type 1 & rs3818361 & 1 & $\mathrm{G} / \mathrm{A}$ & 0.26 & 1.17 \\
\hline EPHA1 & Ephrin type-A receptor 1 & rs11767557 & 7 & $\mathrm{~T} / \mathrm{C}$ & 0.20 & 0.89 \\
\hline$M S_{4} A_{4} A$ & $\begin{array}{l}\text { Membrane-spanning 4-domains } \\
\text { subfamily A member 4A }\end{array}$ & rs4938933 & 11 & $\mathrm{~T} / \mathrm{C}$ & 0.50 & 0.88 \\
\hline$M S_{4} A_{4} E$ & $\begin{array}{l}\text { Membrane-spanning 4-domains } \\
\text { subfamily A member } 4 \mathrm{E}\end{array}$ & rs670139 & 11 & $\mathrm{G} / \mathrm{T}$ & 0.34 & 1.08 \\
\hline$M S_{4} A 6 A$ & $\begin{array}{l}\text { Membrane-spanning 4-domains } \\
\text { subfamily A member } 6 \mathrm{~A}\end{array}$ & rs610932 & 11 & $\mathrm{~T} / \mathrm{G}$ & 0.45 & 0.90 \\
\hline$P I C A L M$ & $\begin{array}{l}\text { Phosphatidylinositol-binding clathrin } \\
\text { assembly protein }\end{array}$ & rs3851179 & 11 & $\mathrm{C} / \mathrm{T}$ & 0.41 & 0.88 \\
\hline
\end{tabular}


Additional AD, dementia and cognition SNPs

\begin{tabular}{|c|c|c|c|c|c|}
\hline$B D N F$ & Brain-derived neurotrophic factor & rs6265 & 11 & $\mathrm{C} / \mathrm{T}$ & 0.20 \\
\hline CETP & Cholesteryl ester transfer protein & rs5882 & 16 & $\mathrm{~A} / \mathrm{G}$ & 0.36 \\
\hline$C O M T$ & Catechol O-methyltransferase & rs4680 & 22 & $\mathrm{G} / \mathrm{A}$ & 0.48 \\
\hline$C T N N B L 1$ & Beta-catenin-like protein 1 & rs6125962 & 20 & $\mathrm{~T} / \mathrm{C}$ & 0.60 \\
\hline$F R M D 4 A$ & FERM domain-containing protein $4 \mathrm{~A}$ & rs17314229 & 10 & $\mathrm{C} / \mathrm{T}$ & 0.09 \\
\hline$F R M D 4 A$ & FERM domain-containing protein $4 \mathrm{~A}$ & rs7081208 & 10 & $\mathrm{G} / \mathrm{A}$ & 0.29 \\
\hline Intergenic & - & rs12007229 & $\mathrm{X}$ & $\mathrm{C} / \mathrm{A}$ & 0.12 \\
\hline$L G A L S 3$ & Galectin-3 & rs4644 & 14 & $\mathrm{C} / \mathrm{A}$ & 0.49 \\
\hline MMP12 & Macrophage metalloelastase & rs12808148 & 11 & $\mathrm{~T} / \mathrm{C}$ & 0.20 \\
\hline \multirow[t]{3}{*}{ MTHFD1L } & Methylenetetrahydrofolate & rs11754661 & 6 & $\mathrm{G} / \mathrm{A}$ & 0.07 \\
\hline & dehydrogenase (NADP + dependent) & & & & \\
\hline & 1-like & & & & \\
\hline PAICS & Multifunctional protein ADE2 & rs11549976 & 4 & $\mathrm{~A} / \mathrm{C}$ & 0.08 \\
\hline PDE $7 A$ & $\begin{array}{l}\text { High affinity cAMP-specific 3',5'-cyclic } \\
\text { phosphodiesterase } 7 \mathrm{~A}\end{array}$ & rs10808746 & 8 & $\mathrm{G} / \mathrm{A}$ & 0.48 \\
\hline SNTG1 & Gamma-1-syntrophin & rs16914781 & 8 & $\mathrm{~A} / \mathrm{G}$ & 0.40 \\
\hline SORL1 & Sortilin-related receptor & rs668387 & 11 & $\mathrm{C} / \mathrm{T}$ & 0.48 \\
\hline SPON1 & Spondin-1 & rs11023139 & 11 & $\mathrm{G} / \mathrm{A}$ & 0.06 \\
\hline ZNF224 & Zinc finger protein 224 & rs3746319 & 19 & $\mathrm{G} / \mathrm{A}$ & 0.19 \\
\hline
\end{tabular}


Table 2: SNPs that were genotyped as part of this study

\begin{tabular}{|c|c|c|c|c|c|c|}
\hline Gene & SNP & Chromsome & Alleles ${ }^{\dagger}$ & $\mathrm{MAF}^{\ddagger}$ & Association & Citations \\
\hline$A B C A 7^{*}$ & rs3764650 & 19 & $\mathrm{~T} / \mathrm{G}$ & 0.11 & Alzheimer's disease & Hollingworth et al. (2011) \\
\hline$A D R B 1$ & rs1801253 & 10 & $\mathrm{C} / \mathrm{G}$ & 0.31 & Blood Pressure & Johnson et al. (2011b) Johnson et al. (2011a) \\
\hline$A G T$ & rs2004776 & 1 & $\mathrm{C} / \mathrm{T}$ & 0.26 & Blood Pressure & Johnson et al. (2011b) Johnson et al. (2011a) \\
\hline ARHGAP42 & rs633185 & 11 & $\mathrm{C} / \mathrm{G}$ & 0.30 & Blood Pressure & Bis et al. (2012) \\
\hline$A S T N 2$ & rs7852872 & 9 & $\mathrm{C} / \mathrm{G}$ & 0.39 & Neuroanatomy & Bis et al. (2012) \\
\hline ATP2B1 & rs2681472 & 12 & $\mathrm{~A} / \mathrm{G}$ & 0.12 & Blood Pressure & Levy et al. (2009) \\
\hline$B A G 6$ & rs805303 & 6 & $\mathrm{G} / \mathrm{A}$ & 0.31 & Blood Pressure & Bis et al. (2012) \\
\hline$B D N F^{*}$ & rs6265 & 11 & $\mathrm{C} / \mathrm{T}$ & 0.20 & Cognition & Harris et al. (2006) Mandelman and Grigorenko (2012) \\
\hline$B I N 1^{*}$ & rs744373 & 2 & $\mathrm{~A} / \mathrm{G}$ & 0.31 & Alzheimer's disease & Naj et al. (2011) Seshadri S and et al (2010) \\
\hline$C D 2 A P^{*}$ & rs9296559 & 6 & $\mathrm{~T} / \mathrm{C}$ & 0.27 & Alzheimer's disease & Naj et al. (2011) Hollingworth et al. (2011) \\
\hline CD33* & rs34813869 & 19 & $\mathrm{~A} / \mathrm{G}$ & 0.29 & Alzheimer's disease & Naj et al. (2011) Hollingworth et al. (2011) \\
\hline$C E T P^{*}$ & rs5882 & 16 & $\mathrm{~A} / \mathrm{G}$ & 0.36 & Cognition & Izaks et al. (2012) \\
\hline $\mathrm{CHRNA}_{4}$ & rs1044396 & 20 & $\mathrm{G} / \mathrm{A}$ & 0.42 & Neuroanatomy & Markett et al. (2013) \\
\hline$C L U^{*}$ & rs11136000 & 8 & $\mathrm{C} / \mathrm{T}$ & 0.35 & Alzheimer's disease & Naj et al. (2011) Lambert et al. (2009) \\
\hline$C O M T^{*}$ & rs4680 & 22 & $\mathrm{G} / \mathrm{A}$ & 0.48 & Cognition & Houlihan et al. (2009) Harris et al. (2005) \\
\hline$C R 1^{*}$ & rs3818361 & 1 & $\mathrm{G} / \mathrm{A}$ & 0.26 & Alzheimer's disease & Naj et al. (2011) Lambert et al. (2009) \\
\hline$C S K$ & rs1378942 & 15 & $\mathrm{~A} / \mathrm{C}$ & 0.32 & Blood Pressure & Bis et al. (2012) \\
\hline
\end{tabular}




\begin{tabular}{|c|c|c|c|c|c|c|}
\hline$C T N N B L 1^{*}$ & rs6125962 & 20 & $\mathrm{~T} / \mathrm{C}$ & 0.60 & Cognition & Papassotiropoulos et al. (2013) \\
\hline CYP19A1 & rs700518 & 15 & $\mathrm{C} / \mathrm{T}$ & 0.42 & Neuroanatomy & Bayer et al. (2013) \\
\hline $\mathrm{DPP}_{4}$ & rs6741949 & 2 & $\mathrm{G} / \mathrm{C}$ & 0.43 & Neuroanatomy & Bis et al. (2012) \\
\hline DRD2 & rs6277 & 11 & $\mathrm{~A} / \mathrm{G}$ & 0.47 & Neuroanatomy & Markett et al. (2013) \\
\hline$E P H A 1-A S 1^{*}$ & rs11767557 & 7 & $\mathrm{~T} / \mathrm{C}$ & 0.20 & Alzheimer's disease & Naj et al. (2011) Hollingworth et al. (2011) \\
\hline$F 5$ & rs6703865 & 1 & $\mathrm{G} / \mathrm{A}$ & 0.40 & Neuroanatomy & Melville et al. (2012) \\
\hline FGF5 & rs1458038 & 4 & $\mathrm{C} / \mathrm{T}$ & 0.27 & Blood Pressure & Bis et al. (2012) \\
\hline$F R M D 4 A^{*}$ & rs17314229 & 10 & $\mathrm{C} / \mathrm{T}$ & 0.09 & Alzheimer's disease & Lambert et al. (2013) \\
\hline$F R M D_{4} A$ & rs2446581 & & & & Alzheimer's disease & Lambert et al. (2013) \\
\hline$F R M D 4 A^{*}$ & rs7081208 & 10 & $\mathrm{G} / \mathrm{A}$ & 0.29 & Alzheimer's disease & Lambert et al. (2013) \\
\hline FTO & rs3751812 & 16 & $\mathrm{G} / \mathrm{T}$ & 0.46 & Neuroanatomy & Ho et al. (2010) \\
\hline$G C F C 2$ & rs2298948 & 2 & $\mathrm{~T} / \mathrm{C}$ & 0.33 & Neuroanatomy & Melville et al. (2012) \\
\hline GNAS-EDN3 & rs6015450 & 20 & $\mathrm{~A} / \mathrm{G}$ & 0.07 & Blood Pressure & Bis et al. (2012) \\
\hline GRIN2B & rs10845840 & 12 & $\mathrm{C} / \mathrm{T}$ & 0.46 & Neuroanatomy & Kohannim et al. (2012) \\
\hline$H F E$ & rs1799945 & 6 & $\mathrm{C} / \mathrm{G}$ & 0.18 & Blood Pressure & Bis et al. (2012) \\
\hline Intergenic & rs7294919 & 12 & $\mathrm{~T} / \mathrm{C}$ & 0.10 & Neuroanatomy & Bis et al. (2012) \\
\hline Intergenic & rs11139399 & 9 & $\mathrm{~T} / \mathrm{C}$ & 0.41 & Neuroanatomy & Melville et al. (2012) \\
\hline Intergenic & rs2942354 & 1 & $\mathrm{C} / \mathrm{A}$ & 0.44 & Neuroanatomy & Melville et al. (2012) \\
\hline Intergenic* & rs12007229 & $\mathrm{X}$ & $\mathrm{C} / \mathrm{A}$ & 0.12 & Dementia & Schrijvers et al. (2012) \\
\hline
\end{tabular}




\begin{tabular}{|c|c|c|c|c|c|c|}
\hline$L G A L S 3^{*}$ & rs4644 & 14 & $\mathrm{C} / \mathrm{A}$ & 0.49 & Cognition & Trompet et al. (2012) \\
\hline$M E C P \mathscr{2}$ & rs2239464 & $\mathrm{X}$ & $\mathrm{G} / \mathrm{A}$ & 0.22 & Neuroanatomy & Joyner et al. (2009) \\
\hline$M M P 12^{*}$ & rs12808148 & 11 & $\mathrm{~T} / \mathrm{C}$ & 0.20 & Dementia & Kamboh et al. (2012) \\
\hline$M S 4 A 4 A^{*}$ & rs4938933 & 11 & $\mathrm{~T} / \mathrm{C}$ & 0.50 & Alzheimer's disease & Naj et al. (2011) \\
\hline$M S_{4} A 6 A^{*}$ & rs610932 & 11 & $\mathrm{~T} / \mathrm{G}$ & 0.45 & Alzheimer's disease & Hollingworth et al. (2011) \\
\hline$M S R B 3$ & rs17178006 & 12 & $\mathrm{~T} / \mathrm{G}$ & 0.09 & Neuroanatomy & Bis et al. (2012) \\
\hline$M T H F D 1 L^{*}$ & rs11754661 & 6 & $\mathrm{G} / \mathrm{A}$ & 0.07 & Alzheimer's disease & Beecham et al. (2009) Naj et al. (2011) \\
\hline NTSR 1 & rs4334545 & 20 & $\mathrm{C} / \mathrm{T}$ & 0.29 & Neuroanatomy & Li et al. (2013) \\
\hline$O P R D 1$ & rs678849 & 1 & $\mathrm{~T} / \mathrm{C}$ & 0.47 & Neuroanatomy & Roussotte et al. (2014) \\
\hline$P A I C S^{*}$ & rs11549976 & 4 & $\mathrm{~A} / \mathrm{C}$ & 0.08 & Dementia & Velez et al. (2013) \\
\hline$P A R P 1$ & rs1136410 & 1 & $\mathrm{~A} / \mathrm{G}$ & 0.15 & Neuroanatomy & Nho et al. (2013) \\
\hline$P D E E^{*} A^{*}$ & rs10808746 & 8 & $\mathrm{G} / \mathrm{A}$ & 0.48 & Cognition & De Jager et al. (2012) \\
\hline PICALM* & rs3851179 & 11 & $\mathrm{C} / \mathrm{T}$ & 0.41 & Alzheimer's disease & Naj et al. (2011) Harold et al. (2009) \\
\hline SELP & rs3917836 & 1 & $\mathrm{~T} / \mathrm{C}$ & 0.05 & Neuroanatomy & Melville et al. (2012) \\
\hline
\end{tabular}




\begin{tabular}{llcccll} 
SNTG1* & rs16914781 & 8 & $\mathrm{~A} / \mathrm{G}$ & 0.40 & Dementia & Velez et al. (2013) \\
SORL1* & $\mathrm{rs668387}$ & 11 & $\mathrm{C} / \mathrm{T}$ & 0.48 & Alzheimer's disease & Rogaeva et al. (2007) \\
SPON1 & $\mathrm{rs} 2618516$ & 11 & $\mathrm{C} / \mathrm{T}$ & 0.36 & Neuroanatomy & Jahanshad et al. (2013) \\
SPON1* & $\mathrm{rs} 11023139$ & 11 & $\mathrm{G} / \mathrm{A}$ & 0.06 & Cognition & Sherva et al. (2013) \\
TNF & $\mathrm{rs} 1800629$ & 6 & $\mathrm{G} / \mathrm{A}$ & 0.17 & Neuroanatomy & Baune et al. (2012) \\
TRIM65 & $\mathrm{rs} 3744028$ & 17 & $\mathrm{~T} / \mathrm{C}$ & 0.20 & Neuroanatomy & Fornage et al. (2011) \\
WDR41 & $\mathrm{rs} 163030$ & 5 & $\mathrm{~A} / \mathrm{C}$ & 0.47 & Neuroanatomy & Stein et al. (2011) \\
WIF1 & $\mathrm{rs6581612}$ & 12 & $\mathrm{~A} / \mathrm{C}$ & 0.25 & Neuroanatomy & Bis et al. (2012) \\
ZNF224* & $\mathrm{rs} 3746319$ & 19 & $\mathrm{G} / \mathrm{A}$ & 0.19 & Dementia & Shulman et al. (2010) \\
\hline
\end{tabular}

${ }^{*}$ SNPs included in the current analysis; ${ }^{\dagger}$ Major/Minor Allele; ${ }^{\ddagger}$ Minor Allele Frequence: HapMap-CEU 
Table 3: Frequency of top AD risk SNP genotypes and alleles at baseline

\begin{tabular}{|c|c|c|}
\hline & Genotype & $\mathrm{n}(\%)$ \\
\hline \multirow[t]{4}{*}{$A P O E$} & $\mathrm{e} 3 / \mathrm{e} 3$ & $1048(62)$ \\
\hline & $\mathrm{e} 2+$ & $217(12.8)$ \\
\hline & $\mathrm{e} 4+$ & $424(25.1)$ \\
\hline & NA & $0(0)$ \\
\hline \multirow[t]{4}{*}{$A B C A 7$} & $\mathrm{~T} / \mathrm{T}$ & $1394(82.5)$ \\
\hline & $\mathrm{G} / \mathrm{T}$ & $278(16.5)$ \\
\hline & $\mathrm{G} / \mathrm{G}$ & $13(0.8)$ \\
\hline & NA & $4(0.2)$ \\
\hline \multirow[t]{4}{*}{$B I N 1$} & $\mathrm{~A} / \mathrm{A}$ & $856(50.7)$ \\
\hline & $\mathrm{A} / \mathrm{G}$ & $683(40.4)$ \\
\hline & $\mathrm{G} / \mathrm{G}$ & $147(8.7)$ \\
\hline & NA & $3(0.2)$ \\
\hline \multirow[t]{4}{*}{$C D 2 A P$} & $\mathrm{~T} / \mathrm{T}$ & $863(51.1)$ \\
\hline & $\mathrm{C} / \mathrm{T}$ & $667(39.5)$ \\
\hline & $\mathrm{C} / \mathrm{C}$ & $153(9.1)$ \\
\hline & NA & $6(0.4)$ \\
\hline \multirow[t]{4}{*}{ CD33 } & $\mathrm{G} / \mathrm{G}$ & 731 (43.3) \\
\hline & $\mathrm{A} / \mathrm{G}$ & $758(44.9)$ \\
\hline & $\mathrm{A} / \mathrm{A}$ & $191(11.3)$ \\
\hline & $\mathrm{NA}$ & $9(0.5)$ \\
\hline \multirow[t]{4}{*}{$C L U$} & $\mathrm{~T} / \mathrm{T}$ & $595(35.2)$ \\
\hline & $\mathrm{C} / \mathrm{T}$ & $814(48.2)$ \\
\hline & $\mathrm{C} / \mathrm{C}$ & $268(15.9)$ \\
\hline & NA & $12(0.7)$ \\
\hline$C R 1$ & $\mathrm{G} / \mathrm{G}$ & $1143(67.7)$ \\
\hline
\end{tabular}




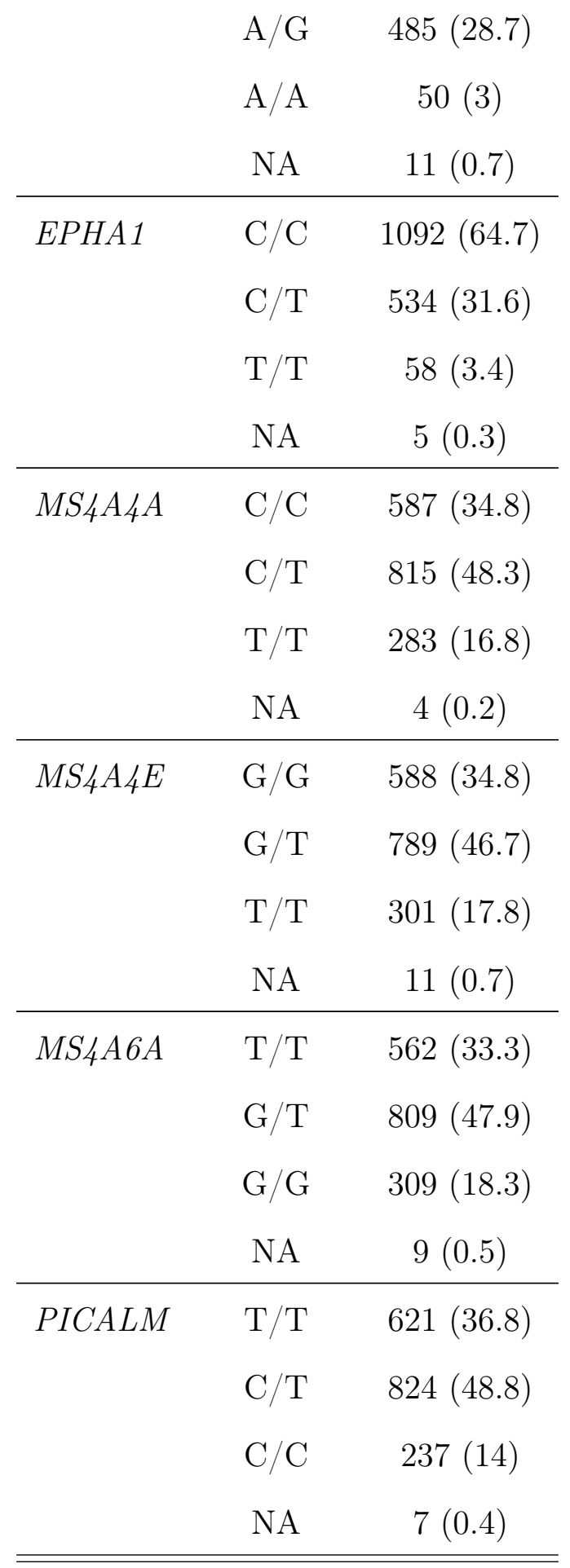

Table 4: Genotype Frequencies of the 16 additional SNPs

Genotype $\mathrm{n}(\%)$




\begin{tabular}{|c|c|c|}
\hline \multirow[t]{4}{*}{$B D N F$} & $\mathrm{C} / \mathrm{C}$ & $1103(65.3)$ \\
\hline & $\mathrm{C} / \mathrm{T}$ & $494(29.2)$ \\
\hline & $\mathrm{T} / \mathrm{T}$ & $50(3)$ \\
\hline & NA & $42(2.5)$ \\
\hline \multirow[t]{4}{*}{ CETP } & $\mathrm{A} / \mathrm{A}$ & $773(45.8)$ \\
\hline & $\mathrm{A} / \mathrm{G}$ & $744(44)$ \\
\hline & $\mathrm{G} / \mathrm{G}$ & $171(10.1)$ \\
\hline & $\mathrm{NA}$ & $1(0.1)$ \\
\hline \multirow[t]{4}{*}{ COMT } & $\mathrm{G} / \mathrm{G}$ & $407(24.1)$ \\
\hline & $\mathrm{A} / \mathrm{G}$ & $827(49)$ \\
\hline & $\mathrm{A} / \mathrm{A}$ & $451(26.7)$ \\
\hline & NA & $4(0.2)$ \\
\hline \multirow[t]{4}{*}{ CTNNBL1 } & $\mathrm{T} / \mathrm{T}$ & $1492(88.3)$ \\
\hline & $\mathrm{C} / \mathrm{T}$ & $188(11.1)$ \\
\hline & $\mathrm{C} / \mathrm{C}$ & $7(0.4)$ \\
\hline & $\mathrm{NA}$ & $2(0.1)$ \\
\hline \multirow[t]{4}{*}{$F R M D 4 A(1)$} & $\mathrm{G} / \mathrm{G}$ & $908(53.8)$ \\
\hline & $\mathrm{A} / \mathrm{G}$ & $646(38.2)$ \\
\hline & $\mathrm{A} / \mathrm{A}$ & $122(7.2)$ \\
\hline & NA & $13(0.8)$ \\
\hline \multirow[t]{4}{*}{$F R M D 4 A(2)$} & $\mathrm{C} / \mathrm{C}$ & $1462(86.6)$ \\
\hline & $\mathrm{C} / \mathrm{T}$ & $212(12.6)$ \\
\hline & $\mathrm{T} / \mathrm{T}$ & $7(0.4)$ \\
\hline & NA & $8(0.5)$ \\
\hline \multirow[t]{2}{*}{ Intergenic chrX } & $\mathrm{C} / \mathrm{C}$ & $1524(90.2)$ \\
\hline & $\mathrm{A} / \mathrm{C}$ & $91(5.4)$ \\
\hline
\end{tabular}




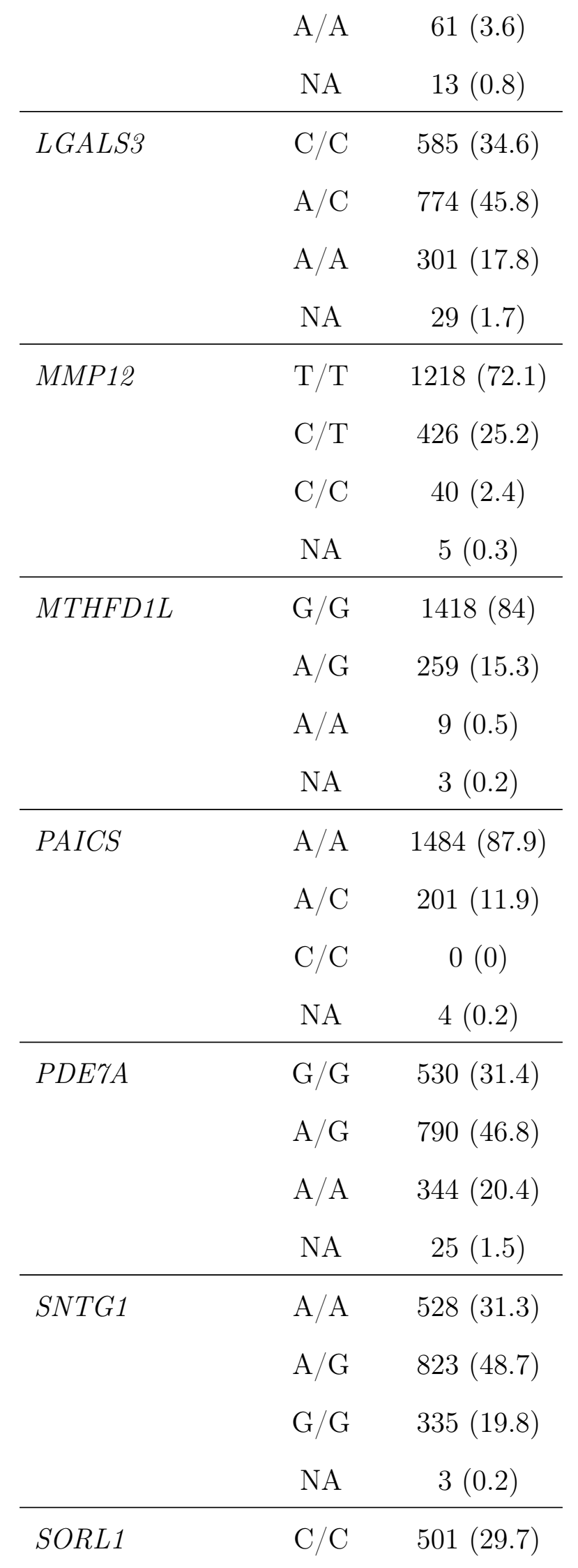




\begin{tabular}{ccc}
$\mathrm{C} / \mathrm{T}$ & $829(49.1)$ \\
$\mathrm{T} / \mathrm{T}$ & $334(19.8)$ \\
$\mathrm{NA}$ & $25(1.5)$ \\
\hline SPON1 & $\mathrm{G} / \mathrm{G}$ & $1506(89.2)$ \\
& $\mathrm{A} / \mathrm{G}$ & $177(10.5)$ \\
$\mathrm{A} / \mathrm{A}$ & $4(0.2)$ \\
& $\mathrm{NA}$ & $2(0.1)$ \\
\hline ZNF224 & $\mathrm{G} / \mathrm{G}$ & $1170(69.3)$ \\
& $\mathrm{A} / \mathrm{G}$ & $469(27.8)$ \\
& $\mathrm{A} / \mathrm{A}$ & $48(2.8)$ \\
$\mathrm{NA}$ & $2(0.1)$ \\
\hline \hline
\end{tabular}

Table 5: Raw cognitive test scores (mean \pm standard deviation)

\begin{tabular}{lllll}
\hline & Episodic Memory & Digits Backwards & Spot-the-Word & $\begin{array}{l}\text { Symbol Digits } \\
\text { Modalities Test }\end{array}$ \\
\hline Wave 1 & $6.62 \pm 2.30$ & $5.05 \pm 2.2$ & $52.57 \pm 5.3$ & $51.11 \pm 8.78$ \\
Wave 2 & $6.51 \pm 2.20$ & $5.21 \pm 2.17$ & $53.32 \pm 4.92$ & $50 \pm 8.93$ \\
Wave 3 & $6.25 \pm 2.18$ & $5.16 \pm 2.15$ & $53.55 \pm 4.86$ & $48.28 \pm 8.97$ \\
\hline
\end{tabular}


Table 6: Person correlations of wave 1 cognitive test scores

\begin{tabular}{|c|c|c|c|c|c|}
\hline & Recall-Immediate & Recall-Delayed & Digits Backwards & Spot-the-Word & $\begin{array}{l}\text { Symbol Digits } \\
\text { Modalities Test }\end{array}$ \\
\hline Recall-Immedite & 1 & & & & \\
\hline Recall-Delayed & $0.84^{* * *}$ & 1 & & & \\
\hline Digits Backwards & $0.24^{* * *}$ & $0.22^{* * *}$ & 1 & & \\
\hline Spot-the-word & $0.24^{* * *}$ & $0.23^{* * *}$ & $0.32^{* * *}$ & 1 & \\
\hline $\begin{array}{l}\text { Symbol Digits } \\
\text { Modaltiies Test }\end{array}$ & $0.27 * * *$ & $0.28 * * *$ & $0.35 * * *$ & $0.33^{* * *}$ & 1 \\
\hline
\end{tabular}


Table 7: Unconditional Means Models

\begin{tabular}{lcccc}
\hline & Episodic Memory & Digits Backwards & Spot-the-Word & Symbol Digits Modalities Test \\
\hline Intercept & $-0.0004(0.0199)$ & $0.1033(0.0207)^{* * *}$ & $0.1986(0.0206)^{* * *}$ & $0.0156(0.0205)$ \\
\hline Individuals & 1689 & 1689 & 1684 & 1688 \\
Variance: Intercept & 0.5364 & 0.6051 & 0.6723 & 0.6338 \\
Variance: Residual & 0.3732 & 0.3311 & 0.1053 & 0.2084 \\
Log Likelihood & -5829.9641 & -5665.0670 & -3839.8119 & -4938.9976 \\
\hline${ }^{* * *} p<0.001,{ }^{* *} p<0.01,{ }^{*} p<0.05$ & & &
\end{tabular}


Table 8: Unconditional Growth Models

\begin{tabular}{lcccc}
\hline & Episodic Memory & Digits Backwards & Spot-the-Word & Symbol Digits Modalities Test \\
\hline Intercept & $0.1152(0.0226)^{* * *}$ & $0.0936(0.0232)^{* * *}$ & $0.1385(0.0217)^{* * *}$ & $0.1643(0.0216)^{* * *}$ \\
Time & $-0.0297(0.0027)^{* * *}$ & $0.0026(0.0026)$ & $0.0161(0.0015)^{* * *}$ & $-0.0390(0.0020)^{* * *}$ \\
\hline Individuals & 1689 & 1689 & 1684 & 1688 \\
Variance: Intercept & 0.5774 & 0.6460 & 0.7135 & 0.6447 \\
Variance: Time & 0.0012 & 0.0013 & 0.0008 & 0.0007 \\
Variance: Residual & 0.3384 & 0.3091 & 0.0891 & 0.1714 \\
Log Likelihood & -5763.8218 & -5660.5924 & -3763.6267 & -4737.9415 \\
$F$-test & $123.59^{* * *}$ & 1.02 & $112.96^{* * *}$ & $393.46^{* * *}$ \\
$R_{m}^{2}$ & 0.013 & 0 & 0.004 & 0.023 \\
$R_{c}^{2}$ & 0.63 & 0.67 & 0.885 & 0.798 \\
\hline
\end{tabular}

$F$-test with Kenward-Roger approximation

$R_{m}^{2}$ : Marginal $R^{2}$ statistics; $R_{c}^{2}$ : Conditional $R^{2}$ statistic

${ }^{* * *} p<0.001,{ }^{* *} p<0.01,{ }^{*} p<0.05$ 
Table 9: Covarites

\begin{tabular}{|c|c|c|c|c|}
\hline & Episodic Memory & Digits Backwards & Spot-the-Word & Symbol Digits Modalities Test \\
\hline \multicolumn{5}{|l|}{ Initial Status } \\
\hline Intercept & $-1.3659(0.1239)^{* * *}$ & $-1.1585(0.1302)^{* * *}$ & $-2.0484(0.1130)^{* * *}$ & $-1.2125(0.1210)^{* * *}$ \\
\hline Gender & $0.4761(0.0432)^{* * *}$ & $-0.0550(0.0454)$ & $0.0060(0.0392)$ & $0.1087(0.0422)^{* *}$ \\
\hline Education & $0.0894(0.0084)^{* * *}$ & $0.0913(0.0088)^{* * *}$ & $0.1559(0.0076)^{* * *}$ & $0.0946(0.0082)^{* * *}$ \\
\hline \multicolumn{5}{|l|}{ Rate of Change } \\
\hline Time & $-0.0174(0.0156)$ & $-0.0130(0.0152)$ & $0.0153(0.0090)$ & $-0.0390(0.0115)^{* * *}$ \\
\hline Gender & $0.0034(0.0054)$ & $-0.0051(0.0052)$ & $0.0021(0.0031)$ & $0.0045(0.0040)$ \\
\hline Education & $-0.0010(0.0010)$ & $0.0013(0.0010)$ & $-0.0000(0.0006)$ & $-0.0002(0.0008)$ \\
\hline Individuals & 1689 & 1689 & 1684 & 1688 \\
\hline Variance: Intercept & 0.4849 & 0.5851 & 0.5525 & 0.5870 \\
\hline Variance: Time & 0.0012 & 0.0012 & 0.0008 & 0.0008 \\
\hline Variance: Residual & 0.3380 & 0.3106 & 0.0890 & 0.1709 \\
\hline Log Likelihood & -5638.9910 & -5577.8450 & -3546.1752 & -4664.8600 \\
\hline$F$-test & $67.07^{* * *}$ & $43.28^{* * *}$ & $123.58^{* * *}$ & $38.03^{* * *}$ \\
\hline$R_{m}^{2}$ & 0.112 & 0.071 & 0.21 & 0.091 \\
\hline$R_{c}^{2}$ & 0.629 & 0.669 & 0.885 & 0.798 \\
\hline
\end{tabular}

$F$-test with Kenward-Roger approximation

$R_{m}^{2}$ : Marginal $R^{2}$ statistics; $R_{c}^{2}$ : Conditional $R^{2}$ statistic

${ }^{* * *} p<0.001,{ }^{* *} p<0.01,{ }^{*} p<0.05$ 
Table 10: $A P O E$

\begin{tabular}{lcccc}
\hline & Episodic Memory & Digits Backwards & Spot-the-Word & Symbol Digits Modalities Test \\
\hline Initial Status & & & & \\
$\quad$ Intercept & $-1.3727(0.1251)^{* * *}$ & $-1.1629(0.1315)^{* * *}$ & $-2.0618(0.1140)^{* * *}$ & $-1.2041(0.1221)^{* * *}$ \\
Gender & $0.4762(0.0432)^{* * *}$ & $-0.0550(0.0454)$ & $0.0065(0.0392)$ & $0.1084(0.0422)^{*}$ \\
Education & $0.0894(0.0084)^{* * *}$ & $0.0913(0.0088)^{* * *}$ & $0.1559(0.0076)^{* * *}$ & $0.0946(0.0082)^{* * *}$ \\
APOE e2 & $-0.0225(0.0657)$ & $0.0100(0.0691)$ & $0.0590(0.0594)$ & $-0.0419(0.0640)$ \\
APOE e4 & $0.0346(0.0502)$ & $0.0151(0.0528)$ & $0.0217(0.0457)$ & $-0.0109(0.0492)$ \\
Rate of Change & & & & $-0.0384(0.0116)^{* * *}$ \\
Time & $-0.0121(0.0157)$ & $-0.0177(0.0153)$ & $0.0160(0.0091)$ & $0.0045(0.0040)$ \\
Gender & $0.0033(0.0054)$ & $-0.0047(0.0052)$ & $0.0020(0.0031)$ & $-0.0001(0.0008)$ \\
Education & $-0.0010(0.0010)$ & $0.0013(0.0010)$ & $-0.0000(0.0006)$ & $0.0050(0.0060)$ \\
APOE e2 & $-0.0029(0.0082)$ & $0.0149(0.0079)$ & $-0.0019(0.0046)$ & $-0.0056(0.0046)$ \\
APOE e4 & $-0.0193(0.0063)^{* *}$ & $0.0095(0.0061)$ & $-0.0019(0.0036)$ & 1688 \\
\hline Individuals & 1689 & 1689 & 1684 & 0.5870 \\
Variance: Intercept & 0.4847 & 0.5846 & 0.5521 & 0.0008 \\
Variance: Time & 0.0012 & 0.0011 & 0.0008 & 0.1708 \\
Variance: Residual & 0.3377 & 0.3112 & 0.0890 & -4663.2460 \\
Log Likelihood & -5633.7563 & -5574.2830 & -3545.5803 & 0.80 \\
$F$-test & $2.61^{*}$ & 1.78 & 0.30 & 0.091 \\
$R_{m}^{2}$ & 0.114 & 0.072 & 0.21 & 0.798 \\
$R_{c}^{2}$ & 0.63 & 0.668 & 0.885 & \\
\hline$F$-t & & & &
\end{tabular}

$F$-test with Kenward-Roger approximation

$R_{m}^{2}$ : Marginal $R^{2}$ statistics; $R_{c}^{2}$ : Conditional $R^{2}$ statistic

${ }^{* * *} p<0.001,{ }^{* *} p<0.01,{ }^{*} p<0.05$ 
Table 11: $A B C A$ r

\begin{tabular}{|c|c|c|c|c|}
\hline & Episodic Memory & Digits Backwards & Spot-the-Word & Symbol Digits Modalities Test \\
\hline \multicolumn{5}{|l|}{ Initial Status } \\
\hline Intercept & $-1.3615(0.1251)^{* * *}$ & $-1.1630(0.1318)^{* * *}$ & $-2.0545(0.1142)^{* * *}$ & $-1.1973(0.1223)^{* * *}$ \\
\hline Gender & $0.4805(0.0432)^{* * *}$ & $-0.0577(0.0455)$ & $0.0092(0.0393)$ & $0.1077(0.0422)^{*}$ \\
\hline Education & $0.0897(0.0083)^{* * *}$ & $0.0912(0.0088)^{* * *}$ & $0.1558(0.0076)^{* * *}$ & $0.0947(0.0082)^{* * *}$ \\
\hline$A P O E \mathrm{e} 2$ & $-0.0184(0.0656)$ & $0.0092(0.0691)$ & $0.0608(0.0594)$ & $-0.0421(0.0640)$ \\
\hline$A P O E \mathrm{e} 4$ & $0.0332(0.0502)$ & $0.0117(0.0529)$ & $0.0234(0.0457)$ & $-0.0133(0.0492)$ \\
\hline$A B C A 7$ & $-0.1174(0.0563)^{*}$ & $0.0198(0.0593)$ & $-0.0507(0.0513)$ & $-0.0404(0.0551)$ \\
\hline \multicolumn{5}{|l|}{ Rate of Change } \\
\hline Time & $-0.0118(0.0157)$ & $-0.0185(0.0153)$ & $0.0160(0.0091)$ & $-0.0390(0.0116)^{* * *}$ \\
\hline Gender & $0.0031(0.0054)$ & $-0.0049(0.0052)$ & $0.0020(0.0031)$ & $0.0044(0.0040)$ \\
\hline Education & $-0.0010(0.0010)$ & $0.0013(0.0010)$ & $-0.0000(0.0006)$ & $-0.0002(0.0008)$ \\
\hline$A P O E \mathrm{e} 2$ & $-0.0032(0.0082)$ & $0.0148(0.0079)$ & $-0.0019(0.0046)$ & $0.0048(0.0060)$ \\
\hline$A P O E \mathrm{e} 4$ & $-0.0194(0.0063)^{* *}$ & $0.0095(0.0061)$ & $-0.0019(0.0036)$ & $-0.0054(0.0047)$ \\
\hline$A B C A 7$ & $-0.0003(0.0070)$ & $0.0043(0.0068)$ & $-0.0002(0.0040)$ & $0.0064(0.0051)$ \\
\hline Individuals & 1685 & 1685 & 1680 & 1684 \\
\hline Variance: Intercept & 0.4816 & 0.5845 & 0.5515 & 0.5853 \\
\hline Variance: Time & 0.0012 & 0.0011 & 0.0008 & 0.0008 \\
\hline Variance: Residual & 0.3378 & 0.3111 & 0.0891 & 0.1710 \\
\hline Log Likelihood & -5618.7534 & -5562.8453 & -3539.6808 & -4652.9621 \\
\hline$F$-test & 2.93 & 0.46 & 0.59 & 0.84 \\
\hline$R_{m}^{2}$ & 0.116 & 0.072 & 0.211 & 0.092 \\
\hline$R_{c}^{2}$ & 0.63 & 0.668 & 0.885 & 0.798 \\
\hline
\end{tabular}

$F$-test with Kenward-Roger approximation

$R_{m}^{2}$ : Marginal $R^{2}$ statistics; $R_{c}^{2}$ : Conditional $R^{2}$ statistic

${ }^{* * *} p<0.001,{ }^{* *} p<0.01,{ }^{*} p<0.05$ 
Table 12: BIN1

\begin{tabular}{|c|c|c|c|c|}
\hline & Episodic Memory & Digits Backwards & Spot-the-Word & Symbol Digits Modalities Test \\
\hline \multicolumn{5}{|l|}{ Initial Status } \\
\hline Intercept & $-1.3524(0.1261)^{* * *}$ & $-1.1802(0.1325)^{* * *}$ & $-2.0679(0.1151)^{* * *}$ & $-1.2134(0.1231)^{* * *}$ \\
\hline Gender & $0.4753(0.0432)^{* * *}$ & $-0.0593(0.0454)$ & $0.0066(0.0393)$ & $0.1056(0.0423)^{*}$ \\
\hline Education & $0.0889(0.0084)^{* * *}$ & $0.0900(0.0088)^{* * *}$ & $0.1563(0.0076)^{* * *}$ & $0.0938(0.0082)^{* * *}$ \\
\hline$A P O E \mathrm{e} 2$ & $-0.0207(0.0657)$ & $0.0126(0.0691)$ & $0.0585(0.0595)$ & $-0.0403(0.0640)$ \\
\hline$A P O E \mathrm{e} 4$ & $0.0361(0.0503)$ & $0.0114(0.0529)$ & $0.0234(0.0458)$ & $-0.0126(0.0492)$ \\
\hline$B I N 1$ & $-0.0298(0.0427)$ & $0.0739(0.0449)$ & $-0.0002(0.0388)$ & $0.0409(0.0418)$ \\
\hline \multicolumn{5}{|l|}{ Rate of Change } \\
\hline Time & $-0.0105(0.0158)$ & $-0.0186(0.0154)$ & $0.0170(0.0091)$ & $-0.0386(0.0117)^{* * *}$ \\
\hline Gender & $0.0036(0.0054)$ & $-0.0044(0.0052)$ & $0.0021(0.0031)$ & $0.0047(0.0040)$ \\
\hline Education & $-0.0009(0.0010)$ & $0.0014(0.0010)$ & $-0.0000(0.0006)$ & $-0.0001(0.0008)$ \\
\hline$A P O E \mathrm{e} 2$ & $-0.0030(0.0082)$ & $0.0146(0.0079)$ & $-0.0019(0.0046)$ & $0.0048(0.0060)$ \\
\hline$A P O E \mathrm{e} 4$ & $-0.0190(0.0063)^{* *}$ & $0.0093(0.0061)$ & $-0.0020(0.0036)$ & $-0.0057(0.0047)$ \\
\hline BIN1 & $-0.0061(0.0053)$ & $0.0002(0.0052)$ & $-0.0017(0.0030)$ & $-0.0007(0.0039)$ \\
\hline Individuals & 1686 & 1686 & 1681 & 1685 \\
\hline Variance: Intercept & 0.4838 & 0.5832 & 0.5526 & 0.5867 \\
\hline Variance: Time & 0.0012 & 0.0011 & 0.0008 & 0.0008 \\
\hline Variance: Residual & 0.3380 & 0.3117 & 0.0889 & 0.1708 \\
\hline Log Likelihood & -5624.9056 & -5564.5501 & -3540.2586 & -4655.9700 \\
\hline$F$-test & 1.71 & 1.78 & 0.18 & 0.49 \\
\hline$R_{m}^{2}$ & 0.113 & 0.073 & 0.211 & 0.091 \\
\hline$R_{c}^{2}$ & 0.629 & 0.667 & 0.885 & 0.798 \\
\hline
\end{tabular}

$F$-test with Kenward-Roger approximation

$R_{m}^{2}$ : Marginal $R^{2}$ statistics; $R_{c}^{2}$ : Conditional $R^{2}$ statistic

${ }^{* * *} p<0.001,{ }^{* *} p<0.01,{ }^{*} p<0.05$ 
Table 13: $C D 2 A P$

\begin{tabular}{lcccc}
\hline & Episodic Memory & Digits Backwards & Spot-the-Word & Symbol Digits Modalities Test \\
\hline Initial Status & & & & $-1.2124(0.1228)^{* * *}$ \\
Intercept & $-1.3860(0.1259)^{* * *}$ & $-1.1727(0.1323)^{* * *}$ & $-2.0579(0.1148)^{* * *}$ & $0.1092(0.0423)^{* *}$ \\
Gender & $0.4749(0.0433)^{* * *}$ & $-0.0579(0.0455)$ & $0.0047(0.0393)$ & $0.0949(0.0082)^{* * *}$ \\
Education & $0.0898(0.0084)^{* * *}$ & $0.0909(0.0088)^{* * *}$ & $0.1562(0.0076)^{* * *}$ & $-0.0480(0.0641)$ \\
APOE e & $-0.0247(0.0659)$ & $0.0011(0.0692)$ & $0.0530(0.0596)$ & $-0.0081(0.0493)$ \\
APOE e4 & $0.0328(0.0504)$ & $0.0134(0.0530)$ & $0.0213(0.0459)$ & $0.0048(0.0419)$ \\
CD2AP & $0.0182(0.0429)$ & $0.0322(0.0451)$ & $-0.0162(0.0390)$ & $-0.0385(0.0117)^{* * *}$ \\
Rate of Change & & & & $0.0048(0.0040)$ \\
Time & $-0.0114(0.0158)$ & $-0.0173(0.0154)$ & $0.0165(0.0091)$ & $-0.0001(0.0008)$ \\
Gender & $0.0037(0.0054)$ & $-0.0047(0.0053)$ & $0.0024(0.0031)$ & $0.0059(0.0060)$ \\
Education & $-0.0009(0.0010)$ & $0.0013(0.0010)$ & $0.0000(0.0006)$ & $-0.0057(0.0047)$ \\
APOE e2 & $-0.0030(0.0082)$ & $0.0150(0.0080)$ & $-0.0018(0.0047)$ & $-0.0010(0.0040)$ \\
APOE e4 & $-0.0188(0.0063)^{* *}$ & $0.0096(0.0062)$ & $-0.0013(0.0036)$ & 1682 \\
CD2AP & $-0.0057(0.0054)$ & $-0.0009(0.0052)$ & $-0.0032(0.0031)$ & 0.5858 \\
Individuals & 1683 & 1683 & 1678 & 0.0008 \\
Variance: Intercept & 0.4863 & 0.5841 & 0.5529 & 0.1711 \\
Variance: Time & 0.0012 & 0.0012 & 0.0008 & -4646.5940 \\
Variance: Residual & 0.3355 & 0.3107 & 0.0887 & 0.03 \\
Log Likelihood & -5605.3421 & -5554.3613 & -3533.6684 & 0.092 \\
F-test & 0.56 & 0.27 & 0.91 & 0.798 \\
$R_{m}^{2}$ & 0.115 & 0.072 & 0.211 & 0.885 \\
$R_{c}^{2}$ & 0.632 & 0.668 & &
\end{tabular}

$F$-test with Kenward-Roger approximation

$R_{m}^{2}$ : Marginal $R^{2}$ statistics; $R_{c}^{2}$ : Conditional $R^{2}$ statistic

${ }^{* * *} p<0.001,{ }^{* *} p<0.01,{ }^{*} p<0.05$ 
Table 14: CD33

\begin{tabular}{lcccc}
\hline & Episodic Memory & Digits Backwards & Spot-the-Word & Symbol Digits Modalities Test \\
\hline Initial Status & & & & $-1.2592(0.1369)^{* * *}$ \\
Intercept & $-1.3509(0.1398)^{* * *}$ & $-1.1070(0.1471)^{* * *}$ & $-1.9917(0.1273)^{* * *}$ & $0.1076(0.0424)^{*}$ \\
Gender & $0.4794(0.0432)^{* * *}$ & $-0.0590(0.0455)$ & $0.0022(0.0393)$ & $0.0946(0.0082)^{* * *}$ \\
Education & $0.0892(0.0084)^{* * *}$ & $0.0911(0.0088)^{* * *}$ & $0.1555(0.0076)^{* * *}$ & $-0.0431(0.0642)$ \\
APOE e2 & $-0.0226(0.0657)$ & $0.0047(0.0691)$ & $0.0567(0.0594)$ & $-0.0157(0.0494)$ \\
APOE e4 & $0.0370(0.0503)$ & $0.0162(0.0529)$ & $0.0200(0.0458)$ & $0.0638(0.0657)$ \\
CD33 & $-0.0242(0.0669)$ & $-0.0531(0.0704)$ & $-0.0697(0.0609)$ & $-0.0359(0.0130)^{* *}$ \\
Rate of Change & & & & $0.0042(0.0040)$ \\
Time & $-0.0125(0.0176)$ & $-0.0112(0.0172)$ & $0.0126(0.0101)$ & $-0.0001(0.0008)$ \\
Gender & $0.0030(0.0054)$ & $-0.0047(0.0052)$ & $0.0020(0.0031)$ & $0.0052(0.0060)$ \\
Education & $-0.0010(0.0010)$ & $0.0014(0.0010)$ & $0.0001(0.0006)$ & $-0.0056(0.0047)$ \\
APOE e2 & $-0.0030(0.0082)$ & $0.0146(0.0079)$ & $-0.0019(0.0046)$ & $-0.0029(0.0062)$ \\
APOE e4 & $-0.0190(0.0063)^{* *}$ & $0.0094(0.0061)$ & $-0.0016(0.0036)$ & 1679 \\
CD33 & $0.0004(0.0085)$ & $-0.0086(0.0082)$ & $0.0026(0.0048)$ & 0.5892 \\
Individuals & 1680 & 1680 & 1675 & 0.0008 \\
Variance: Intercept & 0.4829 & 0.5822 & 0.5513 & 0.1713 \\
Variance: Time & 0.0012 & 0.0011 & 0.0008 & -4643.3035 \\
Variance: Residual & 0.3382 & 0.3121 & 0.0891 & 0.48 \\
Log Likelihood & -5604.7889 & -5543.8680 & -3526.3746 & 0.091 \\
F-test & 0.08 & 1.51 & 0.66 & 0.798 \\
$R_{m}^{2}$ & 0.114 & 0.073 & 0.211 & 0.885 \\
$R_{c}^{2}$ & 0.629 & 0.667 & &
\end{tabular}

$F$-test with Kenward-Roger approximation

$R_{m}^{2}$ : Marginal $R^{2}$ statistics; $R_{c}^{2}$ : Conditional $R^{2}$ statistic

${ }^{* * *} p<0.001,{ }^{* *} p<0.01,{ }^{*} p<0.05$ 
Table 15: $C L U$

\begin{tabular}{|c|c|c|c|c|}
\hline & Episodic Memory & Digits Backwards & Spot-the-Word & Symbol Digits Modalities Test \\
\hline \multicolumn{5}{|l|}{ Initial Status } \\
\hline Intercept & $-1.4076(0.1343)^{* * *}$ & $-1.2214(0.1416)^{* * *}$ & $-2.0821(0.1198)^{* * *}$ & $-1.2448(0.1315)^{* * *}$ \\
\hline Gender & $0.4820(0.0433)^{* * *}$ & $-0.0581(0.0456)$ & $0.0035(0.0385)$ & $0.1032(0.0424)^{*}$ \\
\hline Education & $0.0896(0.0084)^{* * *}$ & $0.0914(0.0088)^{* * *}$ & $0.1576(0.0075)^{* * *}$ & $0.0947(0.0082)^{* * *}$ \\
\hline$A P O E \mathrm{e} 2$ & $-0.0235(0.0659)$ & $0.0052(0.0694)$ & $0.0585(0.0583)$ & $-0.0435(0.0643)$ \\
\hline$A P O E \mathrm{e} 4$ & $0.0330(0.0505)$ & $0.0217(0.0532)$ & $0.0394(0.0449)$ & $-0.0104(0.0495)$ \\
\hline$C L U$ & $0.0343(0.0585)$ & $0.0710(0.0616)$ & $-0.0047(0.0519)$ & $0.0506(0.0572)$ \\
\hline \multicolumn{5}{|l|}{ Rate of Change } \\
\hline Time & $-0.0125(0.0169)$ & $-0.0169(0.0164)$ & $0.0164(0.0090)$ & $-0.0418(0.0125)^{* * *}$ \\
\hline Gender & $0.0023(0.0054)$ & $-0.0055(0.0053)$ & $0.0027(0.0029)$ & $0.0047(0.0040)$ \\
\hline Education & $-0.0010(0.0010)$ & $0.0014(0.0010)$ & $-0.0003(0.0006)$ & $-0.0001(0.0008)$ \\
\hline$A P O E \mathrm{e} 2$ & $-0.0024(0.0082)$ & $0.0150(0.0080)$ & $-0.0018(0.0043)$ & $0.0055(0.0060)$ \\
\hline$A P O E \mathrm{e} 4$ & $-0.0182(0.0063)^{* *}$ & $0.0086(0.0062)$ & $-0.0044(0.0034)$ & $-0.0052(0.0047)$ \\
\hline$C L U$ & $0.0004(0.0072)$ & $-0.0013(0.0070)$ & $0.0034(0.0038)$ & $0.0035(0.0053)$ \\
\hline Individuals & 1677 & 1677 & 1672 & 1676 \\
\hline Variance: Intercept & 0.4833 & 0.5868 & 0.5301 & 0.5895 \\
\hline Variance: Time & 0.0012 & 0.0011 & 0.0004 & 0.0008 \\
\hline Variance: Residual & 0.3384 & 0.3112 & 0.0841 & 0.1712 \\
\hline Log Likelihood & -5594.4717 & -5535.4866 & -3361.6835 & -4636.9077 \\
\hline$F$-test & 0.25 & 0.75 & 0.41 & 0.89 \\
\hline$R_{m}^{2}$ & 0.115 & 0.073 & 0.215 & 0.092 \\
\hline$R_{c}^{2}$ & 0.63 & 0.669 & 0.89 & 0.799 \\
\hline
\end{tabular}

$F$-test with Kenward-Roger approximation

$R_{m}^{2}$ : Marginal $R^{2}$ statistics; $R_{c}^{2}$ : Conditional $R^{2}$ statistic

${ }^{* * *} p<0.001,{ }^{* *} p<0.01,{ }^{*} p<0.05$ 
Table 16: $C R 1$

\begin{tabular}{lcccc}
\hline & Episodic Memory & Digits Backwards & Spot-the-Word & Symbol Digits Modalities Test \\
\hline Initial Status & & & & $-1.1752(0.1235)^{* * *}$ \\
Intercept & $-1.3531(0.1266)^{* * *}$ & $-1.1660(0.1333)^{* * *}$ & $-2.0363(0.1155)^{* * *}$ & $0.1134(0.0423)^{* *}$ \\
Gender & $0.4772(0.0433)^{* * *}$ & $-0.0508(0.0456)$ & $0.0058(0.0394)$ & $0.0934(0.0082)^{* * *}$ \\
Education & $0.0888(0.0084)^{* * *}$ & $0.0913(0.0088)^{* * *}$ & $0.1556(0.0076)^{* * *}$ & $-0.0403(0.0643)$ \\
APOE e & $-0.0303(0.0661)$ & $0.0188(0.0696)$ & $0.0663(0.0598)$ & $-0.0147(0.0492)$ \\
APOE e4 & $0.0256(0.0504)$ & $0.0105(0.0530)$ & $0.0196(0.0459)$ & $-0.0481(0.0448)$ \\
CR1 & $-0.0313(0.0459)$ & $0.0067(0.0483)$ & $-0.0647(0.0417)$ & $-0.0386(0.0118)^{* *}$ \\
Rate of Change & & & & $0.0043(0.0040)$ \\
Time & $-0.0084(0.0159)$ & $-0.0153(0.0155)$ & $0.0145(0.0092)$ & $-0.0001(0.0008)$ \\
Gender & $0.0028(0.0054)$ & $-0.0053(0.0052)$ & $0.0024(0.0031)$ & $0.0055(0.0061)$ \\
Education & $-0.0010(0.0010)$ & $0.0012(0.0010)$ & $0.0001(0.0006)$ & $-0.0055(0.0047)$ \\
APOE e2 & $-0.0032(0.0082)$ & $0.0143(0.0080)$ & $-0.0028(0.0047)$ & $-0.0001(0.0042)$ \\
APOE e4 & $-0.0190(0.0063)^{* *}$ & $0.0103(0.0061)$ & $-0.0020(0.0036)$ & 1677 \\
CR1 & $-0.0107(0.0057)$ & $-0.0052(0.0056)$ & $0.0013(0.0033)$ & 0.5844 \\
Individuals & 1678 & 1678 & 1673 & 0.0008 \\
Variance: Intercept & 0.4830 & 0.5866 & 0.5530 & 0.1711 \\
Variance: Time & 0.0011 & 0.0012 & 0.0008 & -4635.0873 \\
Variance: Residual & 0.3383 & 0.3099 & 0.0889 & 0.65 \\
Log Likelihood & -5595.6655 & -5539.2700 & -3522.9848 & 0.091 \\
F-test & $3.46^{*}$ & 0.49 & 1.22 & 0.797 \\
$R_{m}^{2}$ & 0.115 & 0.072 & 0.212 & 0.885 \\
$R_{c}^{2}$ & 0.629 & 0.67 & &
\end{tabular}

$F$-test with Kenward-Roger approximation

$R_{m}^{2}$ : Marginal $R^{2}$ statistics; $R_{c}^{2}$ : Conditional $R^{2}$ statistic

${ }^{* * *} p<0.001,{ }^{* *} p<0.01,{ }^{*} p<0.05$ 
Table 17: EPHA1

\begin{tabular}{lcccc}
\hline & Episodic Memory & Digits Backwards & Spot-the-Word & Symbol Digits Modalities Test \\
\hline Initial Status & & & & \\
Intercept & $-1.4503(0.1732)^{* * *}$ & $-1.2446(0.1824)^{* * *}$ & $-1.9318(0.1576)^{* * *}$ & $-1.0002(0.1694)^{* * *}$ \\
Gender & $0.4776(0.0432)^{* * *}$ & $-0.0518(0.0455)$ & $0.0042(0.0393)$ & $0.1052(0.0423)^{*}$ \\
Education & $0.0898(0.0084)^{* * *}$ & $0.0915(0.0088)^{* * *}$ & $0.1557(0.0076)^{* * *}$ & $0.0939(0.0082)^{* * *}$ \\
APOE e2 & $-0.0210(0.0657)$ & $0.0126(0.0692)$ & $0.0545(0.0595)$ & $-0.0460(0.0641)$ \\
APOE e4 & $0.0303(0.0502)$ & $0.0176(0.0529)$ & $0.0199(0.0458)$ & $-0.0129(0.0493)$ \\
EPHA1 & $0.0740(0.1172)$ & $0.0789(0.1233)$ & $-0.1289(0.1063)$ & $-0.1990(0.1147)$ \\
Rate of Change & & & & $-0.0476(0.0161)^{* *}$ \\
Time & $-0.0004(0.0216)$ & $-0.0020(0.0211)$ & $0.0102(0.0124)$ & $0.0046(0.0040)$ \\
Gender & $0.0031(0.0054)$ & $-0.0051(0.0052)$ & $0.0020(0.0031)$ & $-0.0001(0.0008)$ \\
Education & $-0.0010(0.0010)$ & $0.0012(0.0010)$ & $0.0000(0.0006)$ & $0.0053(0.0060)$ \\
APOE e2 & $-0.0037(0.0082)$ & $0.0147(0.0079)$ & $-0.0019(0.0046)$ & $-0.0054(0.0047)$ \\
APOE e4 & $-0.0196(0.0063)^{* *}$ & $0.0094(0.0061)$ & $-0.0020(0.0036)$ & $0.0091(0.0109)$ \\
EPHA1 & $-0.0120(0.0145)$ & $-0.0151(0.0142)$ & $0.0055(0.0083)$ & 1683 \\
\hline Individuals & 1684 & 1684 & 1679 & 0.5873 \\
Variance: Intercept & 0.4823 & 0.5851 & 0.5522 & 0.0008 \\
Variance: Time & 0.0012 & 0.0011 & 0.0008 & 0.1706 \\
Variance: Residual & 0.3379 & 0.3115 & 0.0889 & -4651.0877 \\
Log Likelihood & -5614.3431 & -5561.8987 & -3531.5628 & 1.54 \\
F-test & 0.37 & 0.57 & 0.76 & 0.092 \\
$R_{m}^{2}$ & 0.114 & 0.072 & 0.212 & 0.799 \\
$R_{c}^{2}$ & 0.629 & 0.668 & 0.885 & \\
\hline$F$-test & & & &
\end{tabular}

$F$-test with Kenward-Roger approximation

$R_{m}^{2}$ : Marginal $R^{2}$ statistics; $R_{c}^{2}$ : Conditional $R^{2}$ statistic

${ }^{* * *} p<0.001,{ }^{* *} p<0.01,{ }^{*} p<0.05$ 
Table 18: MS4A4A

\begin{tabular}{|c|c|c|c|c|}
\hline & Episodic Memory & Digits Backwards & Spot-the-Word & Symbol Digits Modalities Test \\
\hline \multicolumn{5}{|l|}{ Initial Status } \\
\hline Intercept & $-1.3130(0.1346)^{* * *}$ & $-1.1293(0.1415)^{* * *}$ & $-2.1357(0.1225)^{* * *}$ & $-1.2538(0.1312)^{* * *}$ \\
\hline Gender & $0.4738(0.0432)^{* * *}$ & $-0.0535(0.0455)$ & $0.0040(0.0392)$ & $0.1058(0.0422)^{*}$ \\
\hline Education & $0.0891(0.0084)^{* * *}$ & $0.0910(0.0088)^{* * *}$ & $0.1555(0.0076)^{* * *}$ & $0.0946(0.0082)^{* * *}$ \\
\hline$A P O E \mathrm{e} 2$ & $-0.0191(0.0659)$ & $0.0142(0.0692)$ & $0.0543(0.0595)$ & $-0.0337(0.0641)$ \\
\hline$A P O E \mathrm{e} 4$ & $0.0374(0.0503)$ & $0.0149(0.0529)$ & $0.0174(0.0457)$ & $-0.0088(0.0492)$ \\
\hline$M S_{4} A_{4} A$ & $-0.0650(0.0572)$ & $-0.0378(0.0601)$ & $0.0997(0.0518)$ & $0.0604(0.0558)$ \\
\hline \multicolumn{5}{|l|}{ Rate of Change } \\
\hline Time & $-0.0212(0.0168)$ & $-0.0272(0.0163)$ & $0.0134(0.0097)$ & $-0.0348(0.0124)^{* *}$ \\
\hline Gender & $0.0036(0.0054)$ & $-0.0050(0.0052)$ & $0.0021(0.0031)$ & $0.0048(0.0040)$ \\
\hline Education & $-0.0010(0.0010)$ & $0.0013(0.0010)$ & $-0.0000(0.0006)$ & $-0.0002(0.0008)$ \\
\hline$A P O E \mathrm{e} 2$ & $-0.0032(0.0082)$ & $0.0146(0.0079)$ & $-0.0010(0.0046)$ & $0.0048(0.0060)$ \\
\hline$A P O E$ e4 & $-0.0200(0.0063)^{* *}$ & $0.0097(0.0061)$ & $-0.0018(0.0036)$ & $-0.0060(0.0046)$ \\
\hline$M_{4} A_{4} A$ & $0.0105(0.0071)$ & $0.0110(0.0069)$ & $0.0025(0.0040)$ & $-0.0041(0.0052)$ \\
\hline Individuals & 1685 & 1685 & 1680 & 1684 \\
\hline Variance: Intercept & 0.4846 & 0.5845 & 0.5509 & 0.5855 \\
\hline Variance: Time & 0.0011 & 0.0011 & 0.0008 & 0.0007 \\
\hline Variance: Residual & 0.3376 & 0.3115 & 0.0885 & 0.1711 \\
\hline Log Likelihood & -5620.2648 & -5562.5626 & -3527.4762 & -4652.2710 \\
\hline$F$-test & 1.20 & 1.28 & 2.90 & 0.68 \\
\hline$R_{m}^{2}$ & 0.114 & 0.072 & 0.211 & 0.092 \\
\hline$R_{c}^{2}$ & 0.63 & 0.668 & 0.885 & 0.798 \\
\hline
\end{tabular}

$F$-test with Kenward-Roger approximation

$R_{m}^{2}$ : Marginal $R^{2}$ statistics; $R_{c}^{2}$ : Conditional $R^{2}$ statistic

${ }^{* * *} p<0.001,{ }^{* *} p<0.01,{ }^{*} p<0.05$ 
Table 19: MS4A4E

\begin{tabular}{lcccc}
\hline & Episodic Memory & Digits Backwards & Spot-the-Word & Symbol Digits Modalities Test \\
\hline Initial Status & & & & \\
Intercept & $-1.3285(0.1285)^{* * *}$ & $-1.1390(0.1357)^{* * *}$ & $-2.1359(0.1175)^{* * *}$ & $-1.2635(0.1254)^{* * *}$ \\
Gender & $0.4804(0.0432)^{* * *}$ & $-0.0541(0.0456)$ & $0.0093(0.0393)$ & $0.1104(0.0422)^{* *}$ \\
Education & $0.0891(0.0084)^{* * *}$ & $0.0914(0.0088)^{* * *}$ & $0.1559(0.0076)^{* * *}$ & $0.0954(0.0082)^{* * *}$ \\
APOE e2 & $-0.0424(0.0661)$ & $-0.0026(0.0697)$ & $0.0589(0.0599)$ & $-0.0621(0.0643)$ \\
APOE e4 & $0.0357(0.0503)$ & $0.0168(0.0531)$ & $0.0166(0.0458)$ & $-0.0179(0.0492)$ \\
MS4A4E & $-0.0602(0.0447)$ & $-0.0372(0.0472)$ & $0.1107(0.0407)^{* *}$ & $0.0773(0.0437)$ \\
Rate of Change & & & & $-0.0375(0.0120)^{* *}$ \\
Time & $-0.0207(0.0161)$ & $-0.0216(0.0157)$ & $0.0159(0.0094)$ & $0.0046(0.0040)$ \\
Gender & $0.0029(0.0054)$ & $-0.0048(0.0052)$ & $0.0021(0.0031)$ & $-0.0002(0.0008)$ \\
Education & $-0.0011(0.0010)$ & $0.0013(0.0010)$ & $-0.0000(0.0006)$ & $0.0066(0.0061)$ \\
APOE e2 & $-0.0017(0.0082)$ & $0.0163(0.0080)^{*}$ & $-0.0024(0.0047)$ & $-0.0054(0.0047)$ \\
APOE e4 & $-0.0194(0.0063)^{* *}$ & $0.0093(0.0061)$ & $-0.0018(0.0036)$ & $-0.0002(0.0041)$ \\
MS4A4E & $0.0139(0.0056)^{*}$ & $0.0064(0.0054)$ & $0.0007(0.0032)$ & 1677 \\
Individuals & 1678 & 1678 & 1673 & 0.5815 \\
Variance: Intercept & 0.4810 & 0.5874 & 0.5517 & 0.0008 \\
Variance: Time & 0.0011 & 0.0012 & 0.0008 & 0.1714 \\
Variance: Residual & 0.3371 & 0.3105 & 0.0895 & -4632.2743 \\
Log Likelihood & -5590.2460 & -5537.5490 & -3528.4349 & 1.71 \\
$F$-test & $3.10^{*}$ & 0.73 & $4.57^{*}$ & 0.094 \\
$R_{m}^{2}$ & 0.631 & 0.072 & 0.212 & 0.797 \\
$R_{c}^{2}$ & 0.669 & 0.884 & \\
\hline$F$-tist & & & &
\end{tabular}

$F$-test with Kenward-Roger approximation

$R_{m}^{2}$ : Marginal $R^{2}$ statistics; $R_{c}^{2}$ : Conditional $R^{2}$ statistic

${ }^{* * *} p<0.001,{ }^{* *} p<0.01,{ }^{*} p<0.05$ 
Table 20: $M S_{4} A 6 A$

\begin{tabular}{lcccc}
\hline & Episodic Memory & Digits Backwards & Spot-the-Word & Symbol Digits Modalities Test \\
\hline Initial Status & & & & \\
Intercept & $-1.3035(0.1337)^{* * *}$ & $-1.1283(0.1407)^{* * *}$ & $-2.0948(0.1222)^{* * *}$ & $-1.2260(0.1306)^{* * *}$ \\
Gender & $0.4703(0.0432)^{* * *}$ & $-0.0585(0.0455)$ & $0.0062(0.0394)$ & $0.1129(0.0423)^{* *}$ \\
Education & $0.0898(0.0084)^{* * *}$ & $0.0908(0.0088)^{* * *}$ & $0.1561(0.0076)^{* * *}$ & $0.0944(0.0082)^{* * *}$ \\
APOE e2 & $-0.0279(0.0660)$ & $-0.0029(0.0694)$ & $0.0569(0.0598)$ & $-0.0400(0.0643)$ \\
APOE e4 & $0.0287(0.0503)$ & $0.0111(0.0530)$ & $0.0197(0.0459)$ & $-0.0175(0.0493)$ \\
MS4A6A & $-0.0855(0.0552)$ & $-0.0299(0.0580)$ & $0.0374(0.0502)$ & $0.0290(0.0539)$ \\
Rate of Change & & & & $-0.0373(0.0124)^{* *}$ \\
Time & $-0.0200(0.0167)$ & $-0.0265(0.0163)$ & $0.0110(0.0097)$ & $0.0042(0.0040)$ \\
Gender & $0.0038(0.0054)$ & $-0.0046(0.0052)$ & $0.0021(0.0031)$ & $-0.0001(0.0008)$ \\
Education & $-0.0011(0.0010)$ & $0.0013(0.0010)$ & $-0.0000(0.0006)$ & $0.0052(0.0061)$ \\
APOE e2 & $-0.0026(0.0082)$ & $0.0153(0.0080)$ & $-0.0019(0.0047)$ & $-0.0056(0.0047)$ \\
APOE e4 & $-0.0186(0.0063)^{* *}$ & $0.0099(0.0061)$ & $-0.0018(0.0036)$ & $-0.0019(0.0051)$ \\
MS4A6A & $0.0109(0.0069)$ & $0.0110(0.0067)$ & $0.0059(0.0039)$ & 1679 \\
Individuals & 1680 & 1680 & 1675 & 0.5864 \\
Variance: Intercept & 0.4844 & 0.5856 & 0.5541 & 0.0008 \\
Variance: Time & 0.0012 & 0.0011 & 0.0008 & 0.1709 \\
Variance: Residual & 0.3366 & 0.3110 & 0.0894 & -4640.1869 \\
Log Likelihood & -5599.0620 & -5543.4736 & -3533.2931 & 0.17 \\
$F$-test & 1.64 & 1.39 & 2.12 & 0.092 \\
$R_{m}^{2}$ & 0.114 & 0.072 & 0.211 & 0.798 \\
$R_{c}^{2}$ & 0.631 & 0.668 & 0.885 & \\
\hline$F$-t & & & &
\end{tabular}

$F$-test with Kenward-Roger approximation

$R_{m}^{2}$ : Marginal $R^{2}$ statistics; $R_{c}^{2}$ : Conditional $R^{2}$ statistic

${ }^{* * *} p<0.001,{ }^{* *} p<0.01,{ }^{*} p<0.05$ 
Table 21: PICALM

\begin{tabular}{lcccc}
\hline & Episodic Memory & Digits Backwards & Spot-the-Word & Symbol Digits Modalities Test \\
\hline Initial Status & & & & \\
Intercept & $-1.3845(0.1384)^{* * *}$ & $-1.1445(0.1454)^{* * *}$ & $-2.0514(0.1262)^{* * *}$ & $-1.2231(0.1355)^{* * *}$ \\
Gender & $0.4813(0.0432)^{* * *}$ & $-0.0600(0.0454)$ & $0.0061(0.0393)$ & $0.1099(0.0423)^{* *}$ \\
Education & $0.0896(0.0084)^{* * *}$ & $0.0920(0.0088)^{* * *}$ & $0.1561(0.0076)^{* * *}$ & $0.0954(0.0082)^{* * *}$ \\
APOE e2 & $-0.0211(0.0657)$ & $0.0096(0.0690)$ & $0.0578(0.0595)$ & $-0.0438(0.0641)$ \\
APOE e4 & $0.0364(0.0503)$ & $0.0148(0.0528)$ & $0.0146(0.0458)$ & $-0.0151(0.0493)$ \\
PICALM & $0.0071(0.0613)$ & $-0.0311(0.0644)$ & $-0.0151(0.0557)$ & $0.0112(0.0601)$ \\
Rate of Change & & & & $-0.0464(0.0129)^{* * *}$ \\
Time & $-0.0113(0.0175)$ & $-0.0229(0.0169)$ & $0.0128(0.0101)$ & $0.0046(0.0040)$ \\
Gender & $0.0031(0.0054)$ & $-0.0040(0.0052)$ & $0.0021(0.0031)$ & $-0.0001(0.0008)$ \\
Education & $-0.0011(0.0010)$ & $0.0013(0.0010)$ & $0.0000(0.0006)$ & $0.0058(0.0060)$ \\
APOE e2 & $-0.0030(0.0082)$ & $0.0154(0.0079)$ & $-0.0017(0.0046)$ & $-0.0051(0.0046)$ \\
APOE e4 & $-0.0191(0.0063)^{* *}$ & $0.0095(0.0061)$ & $-0.0018(0.0036)$ & $0.0085(0.0056)$ \\
PICALM & $0.0001(0.0076)$ & $0.0061(0.0074)$ & $0.0027(0.0044)$ & 1681 \\
Individuals & 1682 & 1682 & 1677 & 0.5868 \\
Variance: Intercept & 0.4822 & 0.5806 & 0.5514 & 0.0007 \\
Variance: Time & 0.0012 & 0.0011 & 0.0008 & 0.1713 \\
Variance: Residual & 0.3383 & 0.3105 & 0.0892 & -4644.9827 \\
Log Likelihood & -5612.1574 & -5543.3580 & -3532.6002 & 1.41 \\
F-test & 0.01 & 0.35 & 0.19 & 0.093 \\
$R_{m}^{2}$ & 0.115 & 0.073 & 0.212 & 0.798 \\
$R_{c}^{2}$ & 0.629 & 0.668 & 0.885 & \\
\hline$F$-t & & & &
\end{tabular}

$F$-test with Kenward-Roger approximation

$R_{m}^{2}$ : Marginal $R^{2}$ statistics; $R_{c}^{2}$ : Conditional $R^{2}$ statistic

${ }^{* * *} p<0.001,{ }^{* *} p<0.01,{ }^{*} p<0.05$ 
Table 22: SC-GRS with $A P O E$

\begin{tabular}{lcccc}
\hline & Episodic Memory & Digits Backwards & Spot-the-Word & Symbol Digits Modalities Test \\
\hline Initial Status & & & & \\
Intercept & $-1.2860(0.1547)^{* * *}$ & $-1.1407(0.1634)^{* * *}$ & $-2.0800(0.1389)^{* * *}$ & $-1.2928(0.1513)^{* * *}$ \\
Gender & $0.4821(0.0438)^{* * *}$ & $-0.0640(0.0463)$ & $-0.0068(0.0394)$ & $0.1070(0.0430)^{*}$ \\
Education & $0.0900(0.0085)^{* * *}$ & $0.0914(0.0089)^{* * *}$ & $0.1578(0.0076)^{* * *}$ & $0.0950(0.0083)^{* * *}$ \\
SC-GRS & $-0.0090(0.0085)$ & $-0.0013(0.0089)$ & $0.0015(0.0076)$ & $0.0073(0.0083)$ \\
Rate of Change & & & & \\
Time & $-0.0058(0.0195)$ & $-0.0202(0.0190)$ & $0.0203(0.0104)$ & $-0.0403(0.0144)^{* *}$ \\
Gender & $0.0029(0.0055)$ & $-0.0055(0.0054)$ & $0.0037(0.0029)$ & $0.0046(0.0041)$ \\
Education & $-0.0011(0.0011)$ & $0.0014(0.0010)$ & $-0.0001(0.0006)$ & $-0.0002(0.0008)$ \\
SC-GRS & $-0.0010(0.0011)$ & $0.0006(0.0010)$ & $-0.0005(0.0006)$ & $0.0002(0.0008)$ \\
\hline Individuals & 1620 & 1620 & 1615 & 1619 \\
Variance: Intercept & 0.4759 & 0.5857 & 0.5359 & 0.5815 \\
Variance: Time & 0.0012 & 0.0013 & 0.0004 & 0.0008 \\
Variance: Residual & 0.3388 & 0.3096 & 0.0844 & 0.1733 \\
Log Likelihood & -5405.8773 & -5354.5581 & -3259.1441 & -4491.7073 \\
F-test & 1.95 & 0.18 & 0.35 & 0.53 \\
$R_{m}^{2}$ & 0.115 & 0.072 & 0.216 & 0.092 \\
$R_{c}^{2}$ & 0.628 & 0.671 & 0.89 & 0.796 \\
\hline
\end{tabular}

$F$-test with Kenward-Roger approximation

$R_{m}^{2}$ : Marginal $R^{2}$ statistics; $R_{c}^{2}$ : Conditional $R^{2}$ statistic

${ }^{* * *} p<0.001,{ }^{* *} p<0.01,{ }^{*} p<0.05$ 
Table 23: OR-GRS with $A P O E$

\begin{tabular}{lcccc}
\hline & Episodic Memory & Digits Backwards & Spot-the-Word & Symbol Digits Modalities Test \\
\hline Initial Status & & & & $-1.2258(0.1285)^{* * *}$ \\
$\quad$ Intercept & $-1.3818(0.1312)^{* * *}$ & $-1.1511(0.1388)^{* * *}$ & $-2.0627(0.1183)^{* * *}$ & $0.1084(0.0430)^{*}$ \\
Gender & $0.4805(0.0438)^{* * *}$ & $-0.0642(0.0463)$ & $-0.0064(0.0393)$ & $0.0948(0.0083)^{* * *}$ \\
Education & $0.0901(0.0085)^{* * *}$ & $0.0914(0.0089)^{* * *}$ & $0.1578(0.0076)^{* * *}$ & $0.0075(0.0266)$ \\
OR-GRS & $-0.00001(0.0271)$ & $-0.0027(0.0287)$ & $-0.0010(0.0243)$ & $-0.0335(0.0122)^{* *}$ \\
Rate of Change & & & & $0.0048(0.0041)$ \\
$\quad$ Time & $-0.0039(0.0165)$ & $-0.0162(0.0161)$ & $0.0179(0.0089)^{*}$ & $-0.0002(0.0008)$ \\
Gender & $0.0031(0.0055)$ & $-0.0054(0.0054)$ & $0.0037(0.0029)$ & $-0.0036(0.0025)$ \\
Education & $-0.0011(0.0011)$ & $0.0014(0.0010)$ & $-0.0001(0.0006)$ & 1619 \\
OR-GRS & $-0.0086(0.0034)^{*}$ & $0.0016(0.0033)$ & $-0.0018(0.0018)$ & 0.5820 \\
\hline Individuals & 1620 & 1620 & 1615 & 0.0007 \\
Variance: Intercept & 0.4764 & 0.5857 & 0.5359 & 0.1732 \\
Variance: Time & 0.0012 & 0.0013 & 0.0004 & -4491.2292 \\
Variance: Residual & 0.3387 & 0.3097 & 0.0844 & 1.01 \\
Log Likelihood & -5403.6228 & -5354.6026 & -3258.9115 & 0.092 \\
$F$-test & $4.20^{*}$ & 0.13 & 0.58 & 0.796 \\
$R_{m}^{2}$ & 0.116 & 0.072 & 0.216 & 0.89 \\
$R_{c}^{2}$ & 0.628 & 0.671 & & \\
\hline
\end{tabular}

$F$-test with Kenward-Roger approximation

$R_{m}^{2}$ : Marginal $R^{2}$ statistics; $R_{c}^{2}$ : Conditional $R^{2}$ statistic

${ }^{* * *} p<0.001,{ }^{* *} p<0.01,{ }^{*} p<0.05$ 
Table 24: EV-GRS with $A P O E$

\begin{tabular}{lcccc}
\hline & Episodic Memory & Digits Backwards & Spot-the-Word & Symbol Digits Modalities Test \\
\hline Initial Status & & & & $-1.2333(0.1318)^{* * *}$ \\
$\quad$ Intercept & $-1.3751(0.1346)^{* * *}$ & $-1.1487(0.1424)^{* * *}$ & $-2.0676(0.1213)^{* * *}$ & $0.1082(0.0430)^{*}$ \\
Gender & $0.4807(0.0438)^{* * *}$ & $-0.0642(0.0463)$ & $-0.0065(0.0393)$ & $0.0949(0.0083)^{* * *}$ \\
Education & $0.0901(0.0085)^{* * *}$ & $0.0914(0.0089)^{* * *}$ & $0.1578(0.0076)^{* * *}$ & $0.0200(0.0528)$ \\
EV-GRS & $-0.0075(0.0539)$ & $-0.0068(0.0570)$ & $0.0037(0.0483)$ & $-0.0329(0.0126)^{* *}$ \\
Rate of Change & & & & $0.0048(0.0041)$ \\
Time & $-0.0007(0.0170)$ & $-0.0175(0.0165)$ & $0.0189(0.0091)^{*}$ & $-0.0002(0.0008)$ \\
Gender & $0.0031(0.0055)$ & $-0.0054(0.0054)$ & $0.0037(0.0029)$ & $-0.0062(0.0050)$ \\
Education & $-0.0011(0.0011)$ & $0.0014(0.0010)$ & $-0.0001(0.0006)$ & 1619 \\
EV-GRS & $-0.0171(0.0068)^{*}$ & $0.0040(0.0066)$ & $-0.0039(0.0036)$ & 0.5819 \\
\hline Individuals & 1620 & 1620 & 1615 & 0.0007 \\
Variance: Intercept & 0.4764 & 0.5856 & 0.5359 & 0.1733 \\
Variance: Time & 0.0012 & 0.0013 & 0.0004 & -4491.4865 \\
Variance: Residual & 0.3387 & 0.3097 & 0.0844 & 0.75 \\
Log Likelihood & -5403.3829 & -5354.5325 & -3258.8489 & 0.092 \\
$F$-test & $4.44^{*}$ & 0.20 & 0.64 & 0.796 \\
$R_{m}^{2}$ & 0.116 & 0.072 & 0.216 & 0.89 \\
$R_{c}^{2}$ & 0.628 & 0.671 & & \\
\hline
\end{tabular}

$F$-test with Kenward-Roger approximation

$R_{m}^{2}$ : Marginal $R^{2}$ statistics; $R_{c}^{2}$ : Conditional $R^{2}$ statistic

${ }^{* * *} p<0.001,{ }^{* *} p<0.01,{ }^{*} p<0.05$ 
Table 25: SC-GRS without APOE

\begin{tabular}{lcccc}
\hline & Episodic Memory & Digits Backwards & Spot-the-Word & Symbol Digits Modalities Test \\
\hline Initial Status & & & & \\
Intercept & $-1.2759(0.1548)^{* * *}$ & $-1.1455(0.1635)^{* * *}$ & $-2.0759(0.1390)^{* * *}$ & $-1.3005(0.1514)^{* * *}$ \\
Gender & $0.4823(0.0438)^{* * *}$ & $-0.0641(0.0463)$ & $-0.0067(0.0394)$ & $0.1069(0.0430)^{*}$ \\
Education & $0.0899(0.0085)^{* * *}$ & $0.0914(0.0089)^{* * *}$ & $0.1578(0.0076)^{* * *}$ & $0.0950(0.0083)^{* * *}$ \\
SC-GRS & $-0.0101(0.0087)$ & $-0.0009(0.0092)$ & $0.0011(0.0077)$ & $0.0082(0.0085)$ \\
Rate of Change & & & & $-0.0428(0.0144)^{* *}$ \\
Time & $-0.0116(0.0195)$ & $-0.0182(0.0190)$ & $0.0192(0.0104)$ & $0.0045(0.0041)$ \\
Gender & $0.0028(0.0055)$ & $-0.0054(0.0054)$ & $0.0037(0.0029)$ & $-0.0002(0.0008)$ \\
Education & $-0.0011(0.0011)$ & $0.0014(0.0010)$ & $-0.0001(0.0006)$ & $0.0004(0.0008)$ \\
SC-GRS & $-0.0004(0.0011)$ & $0.0004(0.0011)$ & $-0.0004(0.0006)$ & 1619 \\
Individuals & 1620 & 1620 & 1615 & 0.5814 \\
Variance: Intercept & 0.4758 & 0.5858 & 0.5359 & 0.0008 \\
Variance: Time & 0.0012 & 0.0013 & 0.0004 & 0.1733 \\
Variance: Residual & 0.3388 & 0.3096 & 0.0844 & -4491.3769 \\
Log Likelihood & -5406.4959 & -5354.6540 & -3259.2813 & 0.86 \\
$F_{\text {-test }}^{R_{m}^{2}}$ & 1.33 & 0.08 & 0.21 & 0.092 \\
$R_{c}^{2}$ & 0.115 & 0.072 & 0.216 & 0.796 \\
\hline F-tst & 0.628 & 0.671 & 0.89 & \\
\hline
\end{tabular}

$F$-test with Kenward-Roger approximation

$R_{m}^{2}$ : Marginal $R^{2}$ statistics; $R_{c}^{2}$ : Conditional $R^{2}$ statistic

${ }^{* * *} p<0.001,{ }^{* *} p<0.01,{ }^{*} p<0.05$ 
Table 26: OR-GRS without $A P O E$

\begin{tabular}{lcccc}
\hline & Episodic Memory & Digits Backwards & Spot-the-Word & Symbol Digits Modalities Test \\
\hline Initial Status & & & & \\
Intercept & $-1.2465(0.1542)^{* * *}$ & $-1.1698(0.1630)^{* * *}$ & $-2.0526(0.1385)^{* * *}$ & $-1.2770(0.1509)^{* * *}$ \\
Gender & $0.4834(0.0438)^{* * *}$ & $-0.0646(0.0463)$ & $-0.0062(0.0394)$ & $0.1070(0.0430)^{*}$ \\
Education & $0.0900(0.0085)^{* * *}$ & $0.0914(0.0089)^{* * *}$ & $0.1577(0.0076)^{* * *}$ & $0.0949(0.0083)^{* * *}$ \\
OR-GRS & $-0.1116(0.0740)$ & $0.0123(0.0781)$ & $-0.0096(0.0661)$ & $0.0513(0.0723)$ \\
Rate of Change & & & & $-0.0451(0.0144)^{* *}$ \\
Time & $-0.0074(0.0195)$ & $-0.0172(0.0190)$ & $0.0196(0.0104)$ & $0.0044(0.0041)$ \\
Gender & $0.0030(0.0055)$ & $-0.0054(0.0054)$ & $0.0037(0.0029)$ & $-0.0002(0.0008)$ \\
Education & $-0.0011(0.0011)$ & $0.0014(0.0010)$ & $-0.0001(0.0006)$ & $0.0054(0.0069)$ \\
OR-GRS & $-0.0072(0.0093)$ & $0.0027(0.0091)$ & $-0.0035(0.0049)$ & 1619 \\
Individuals & 1620 & 1620 & 1615 & 0.5816 \\
Variance: Intercept & 0.4754 & 0.5858 & 0.5359 & 0.0007 \\
Variance: Time & 0.0012 & 0.0013 & 0.0004 & 0.1733 \\
Variance: Residual & 0.3389 & 0.3096 & -3259.1612 & -4491.3982 \\
Log Likelihood & -5405.1357 & -5354.6316 & 0.33 & 0.84 \\
F-test & 2.69 & 0.10 & 0.216 & 0.092 \\
$R_{m}^{2}$ & 0.116 & 0.072 & 0.89 & 0.796 \\
$R_{c}^{2}$ & 0.627 & 0.671 & \\
\hline
\end{tabular}

$F$-test with Kenward-Roger approximation

$R_{m}^{2}$ : Marginal $R^{2}$ statistics; $R_{c}^{2}$ : Conditional $R^{2}$ statistic

${ }^{* * *} p<0.001,{ }^{* *} p<0.01,{ }^{*} p<0.05$ 
Table 27: EV-GRS without APOE

\begin{tabular}{lcccc}
\hline & Episodic Memory & Digits Backwards & Spot-the-Word & Symbol Digits Modalities Test \\
\hline Initial Status & & & & $-1.2921(0.1509)^{* * *}$ \\
$\quad$ Intercept & $-1.2639(0.1542)^{* * *}$ & $-1.1630(0.1630)^{* * *}$ & $-2.0674(0.1385)^{* * *}$ & $0.1068(0.0430)^{*}$ \\
Gender & $0.4828(0.0438)^{* * *}$ & $-0.0644(0.0463)$ & $-0.0065(0.0394)$ & $0.0950(0.0083)^{* * *}$ \\
Education & $0.0900(0.0085)^{* * *}$ & $0.0914(0.0089)^{* * *}$ & $0.1578(0.0076)^{* * *}$ & $0.0972(0.1105)$ \\
EV-GRS & $-0.1485(0.1130)$ & $0.0102(0.1194)$ & $0.0040(0.1009)$ & $-0.0442(0.0144)^{* *}$ \\
Rate of Change & & & & $0.0045(0.0041)$ \\
$\quad$ Time & $-0.0081(0.0195)$ & $-0.0171(0.0190)$ & $0.0196(0.0104)$ & $-0.0002(0.0008)$ \\
Gender & $0.0029(0.0055)$ & $-0.0054(0.0054)$ & $0.0037(0.0029)$ & $0.0073(0.0105)$ \\
Education & $-0.0011(0.0011)$ & $0.0014(0.0010)$ & $-0.0001(0.0006)$ & 1619 \\
EV-GRS & $-0.0102(0.0143)$ & $0.0041(0.0139)$ & $-0.0054(0.0075)$ & 0.5815 \\
\hline Individuals & 1620 & 1620 & 1615 & 0.0007 \\
Variance: Intercept & 0.4756 & 0.5858 & 0.5359 & 0.1733 \\
Variance: Time & 0.0012 & 0.0013 & 0.0004 & -4491.3083 \\
Variance: Residual & 0.3389 & 0.3096 & 0.0844 & 0.93 \\
Log Likelihood & -5405.7147 & -5354.6601 & -3259.2109 & 0.092 \\
\hline F-test & 2.11 & 0.07 & 0.28 & 0.796 \\
$R_{m}^{2}$ & 0.116 & 0.072 & 0.216 & 0.89 \\
$R_{c}^{2}$ & 0.628 & 0.671 & & \\
\hline
\end{tabular}

$F$-test with Kenward-Roger approximation

$R_{m}^{2}$ : Marginal $R^{2}$ statistics; $R_{c}^{2}$ : Conditional $R^{2}$ statistic

${ }^{* * *} p<0.001,{ }^{* *} p<0.01,{ }^{*} p<0.05$ 
Table 28: BDNF

\begin{tabular}{|c|c|c|c|c|}
\hline & Episodic Memory & Digits Backwards & Spot-the-Word & Symbol Digits Modalities Test \\
\hline \multicolumn{5}{|l|}{ Initial Status } \\
\hline Intercept & $-1.3631(0.1266)^{* * *}$ & $-1.1842(0.1325)^{* * *}$ & $-2.0707(0.1161)^{* * *}$ & $-1.2047(0.1235)^{* * *}$ \\
\hline Gender & $0.4702(0.0436)^{* * *}$ & $-0.0546(0.0456)$ & $-0.0022(0.0398)$ & $0.1019(0.0425)^{*}$ \\
\hline Education & $0.0886(0.0084)^{* * *}$ & $0.0943(0.0088)^{* * *}$ & $0.1569(0.0077)^{* * *}$ & $0.0938(0.0082)^{* * *}$ \\
\hline$A P O E \mathrm{e} 2$ & $-0.0256(0.0668)$ & $-0.0015(0.0698)$ & $0.0689(0.0607)$ & $-0.0600(0.0649)$ \\
\hline$A P O E \mathrm{e} 4$ & $0.0404(0.0508)$ & $0.0348(0.0531)$ & $0.0232(0.0464)$ & $-0.0187(0.0496)$ \\
\hline$B D N F$ & $0.0019(0.0460)$ & $-0.0951(0.0481)^{*}$ & $-0.0213(0.0418)$ & $0.0451(0.0448)$ \\
\hline \multicolumn{5}{|l|}{ Rate of Change } \\
\hline Time & $-0.0117(0.0160)$ & $-0.0132(0.0155)$ & $0.0171(0.0093)$ & $-0.0427(0.0118)^{* * *}$ \\
\hline Gender & $0.0032(0.0055)$ & $-0.0042(0.0053)$ & $0.0019(0.0031)$ & $0.0052(0.0040)$ \\
\hline Education & $-0.0008(0.0011)$ & $0.0011(0.0010)$ & $0.0000(0.0006)$ & $0.0001(0.0008)$ \\
\hline$A P O E \mathrm{e} 2$ & $-0.0015(0.0083)$ & $0.0156(0.0081)$ & $-0.0033(0.0047)$ & $0.0058(0.0061)$ \\
\hline$A P O E \mathrm{e} 4$ & $-0.0197(0.0064)^{* *}$ & $0.0076(0.0062)$ & $-0.0016(0.0037)$ & $-0.0059(0.0047)$ \\
\hline$B D N F$ & $-0.0082(0.0058)$ & $-0.0028(0.0056)$ & $-0.0048(0.0033)$ & $0.0006(0.0042)$ \\
\hline Individuals & 1647 & 1647 & 1642 & 1646 \\
\hline Variance: Intercept & 0.4789 & 0.5723 & 0.5538 & 0.5802 \\
\hline Variance: Time & 0.0012 & 0.0011 & 0.0008 & 0.0008 \\
\hline Variance: Residual & 0.3392 & 0.3108 & 0.0903 & 0.1711 \\
\hline Log Likelihood & -5495.2052 & -5422.6478 & -3473.1845 & -4545.2925 \\
\hline$F$-test & 1.31 & $3.25^{*}$ & 1.75 & 0.64 \\
\hline$R_{m}^{2}$ & 0.113 & 0.077 & 0.214 & 0.093 \\
\hline$R_{c}^{2}$ & 0.626 & 0.668 & 0.884 & 0.797 \\
\hline
\end{tabular}

$F$-test with Kenward-Roger approximation

$R_{m}^{2}$ : Marginal $R^{2}$ statistics; $R_{c}^{2}$ : Conditional $R^{2}$ statistic

${ }^{* * *} p<0.001,{ }^{* *} p<0.01,{ }^{*} p<0.05$ 
Table 29: CETP

\begin{tabular}{lcccc}
\hline & Episodic Memory & Digits Backwards & Spot-the-Word & Symbol Digits Modalities Test \\
\hline Initial Status & & & & \\
Intercept & $-1.3743(0.1270)^{* * *}$ & $-1.1364(0.1335)^{* * *}$ & $-2.0560(0.1158)^{* * *}$ & $-1.2032(0.1240)^{* * *}$ \\
Gender & $0.4757(0.0432)^{* * *}$ & $-0.0548(0.0454)$ & $0.0055(0.0392)$ & $0.1087(0.0422)^{*}$ \\
Education & $0.0893(0.0084)^{* * *}$ & $0.0914(0.0088)^{* * *}$ & $0.1557(0.0076)^{* * *}$ & $0.0946(0.0082)^{* * *}$ \\
APOE e2 & $-0.0221(0.0658)$ & $0.0089(0.0691)$ & $0.0600(0.0594)$ & $-0.0420(0.0641)$ \\
APOE e4 & $0.0347(0.0503)$ & $0.0166(0.0528)$ & $0.0225(0.0457)$ & $-0.0110(0.0492)$ \\
CETP & $0.0052(0.0428)$ & $-0.0527(0.0450)$ & $-0.0073(0.0389)$ & $-0.0029(0.0419)$ \\
Rate of Change & & & & $-0.0375(0.0118)^{* *}$ \\
Time & $-0.0124(0.0159)$ & $-0.0225(0.0155)$ & $0.0186(0.0092)^{*}$ & $0.0045(0.0040)$ \\
Gender & $0.0035(0.0054)$ & $-0.0046(0.0052)$ & $0.0020(0.0031)$ & $-0.0001(0.0008)$ \\
Education & $-0.0010(0.0010)$ & $0.0013(0.0010)$ & $-0.0000(0.0006)$ & $0.0050(0.0060)$ \\
APOE e2 & $-0.0031(0.0082)$ & $0.0148(0.0079)$ & $-0.0019(0.0046)$ & $-0.0056(0.0047)$ \\
APOE e4 & $-0.0194(0.0063)^{* *}$ & $0.0092(0.0061)$ & $-0.0017(0.0036)$ & $-0.0018(0.0039)$ \\
CETP & $-0.0002(0.0053)$ & $0.0096(0.0052)$ & $-0.0051(0.0030)$ & 1687 \\
Individuals & 1688 & 1688 & 1683 & 0.5874 \\
Variance: Intercept & 0.4850 & 0.5842 & 0.5523 & 0.0008 \\
Variance: Time & 0.0012 & 0.0011 & 0.0008 & 0.1707 \\
Variance: Residual & 0.3377 & 0.3112 & 0.0890 & -4661.8838 \\
Log Likelihood & -5631.4349 & -5570.7825 & -3542.5441 & 0.13 \\
F-test & 0.01 & 1.77 & 1.77 & 0.091 \\
$R_{m}^{2}$ & 0.114 & 0.072 & 0.21 & 0.798 \\
$R_{c}^{2}$ & 0.63 & 0.668 & 0.885 & \\
\hline$F$-t & & & &
\end{tabular}

$F$-test with Kenward-Roger approximation

$R_{m}^{2}$ : Marginal $R^{2}$ statistics; $R_{c}^{2}$ : Conditional $R^{2}$ statistic

${ }^{* * *} p<0.001,{ }^{* *} p<0.01,{ }^{*} p<0.05$ 
Table 30: COMT

\begin{tabular}{lcccc}
\hline & Episodic Memory & Digits Backwards & Spot-the-Word & Symbol Digits Modalities Test \\
\hline Initial Status & & & & $-1.1931(0.1273)^{* * *}$ \\
Intercept & $-1.4256(0.1304)^{* * *}$ & $-1.1862(0.1371)^{* * *}$ & $-2.0834(0.1189)^{* * *}$ & $0.1073(0.0422)^{*}$ \\
Gender & $0.4748(0.0432)^{* * *}$ & $-0.0591(0.0454)$ & $0.0070(0.0393)$ & $0.0941(0.0082)^{* * *}$ \\
Education & $0.0891(0.0084)^{* * *}$ & $0.0914(0.0088)^{* * *}$ & $0.1556(0.0076)^{* * *}$ & $-0.0414(0.0640)$ \\
APOE e2 & $-0.0236(0.0657)$ & $0.0099(0.0691)$ & $0.0583(0.0595)$ & $-0.0093(0.0493)$ \\
APOE e4 & $0.0345(0.0503)$ & $0.0221(0.0529)$ & $0.0188(0.0458)$ & $-0.0054(0.0488)$ \\
COMT & $0.0778(0.0498)$ & $0.0320(0.0524)$ & $0.0329(0.0453)$ & $-0.0320(0.0121)^{* *}$ \\
Rate of Change & & & & $0.0048(0.0040)$ \\
Time & $-0.0005(0.0164)$ & $-0.0150(0.0159)$ & $0.0129(0.0094)$ & $-0.0001(0.0008)$ \\
Gender & $0.0035(0.0054)$ & $-0.0044(0.0052)$ & $0.0019(0.0031)$ & $0.0050(0.0060)$ \\
Education & $-0.0010(0.0010)$ & $0.0013(0.0010)$ & $-0.0000(0.0006)$ & $-0.0058(0.0047)$ \\
APOE e2 & $-0.0029(0.0082)$ & $0.0149(0.0079)$ & $-0.0019(0.0046)$ & $-0.0087(0.0046)$ \\
APOE e4 & $-0.0191(0.0063)^{* *}$ & $0.0091(0.0061)$ & $-0.0019(0.0036)$ & 1684 \\
COMT & $-0.0153(0.0062)^{*}$ & $-0.0032(0.0061)$ & $0.0044(0.0036)$ & 0.5861 \\
Individuals & 1685 & 1685 & 1680 & 0.0008 \\
Variance: Intercept & 0.4852 & 0.5844 & 0.5530 & 0.1712 \\
Variance: Time & 0.0012 & 0.0011 & 0.0008 & -4653.1232 \\
Variance: Residual & 0.3368 & 0.3110 & 0.0891 & 2.09 \\
Log Likelihood & -5619.1436 & -5562.8753 & -3539.5246 & 0.091 \\
$F$-test & $3.09 *$ & 0.22 & 1.57 & 0.797 \\
$R_{m}^{2}$ & 0.114 & 0.072 & 0.21 & 0.885 \\
$R_{c}^{2}$ & 0.631 & 0.668 & & \\
\hline$F$-t & & &
\end{tabular}

$F$-test with Kenward-Roger approximation

$R_{m}^{2}$ : Marginal $R^{2}$ statistics; $R_{c}^{2}$ : Conditional $R^{2}$ statistic

${ }^{* * *} p<0.001,{ }^{* *} p<0.01,{ }^{*} p<0.05$ 
Table 31: CTNNBL1

\begin{tabular}{lcccc}
\hline & Episodic Memory & Digits Backwards & Spot-the-Word & Symbol Digits Modalities Test \\
\hline Initial Status & & & & \\
Intercept & $-1.3654(0.1257)^{* * *}$ & $-1.1679(0.1320)^{* * *}$ & $-2.0683(0.1146)^{* * *}$ & $-1.1903(0.1227)^{* * *}$ \\
Gender & $0.4737(0.0432)^{* * *}$ & $-0.0588(0.0454)$ & $0.0061(0.0392)$ & $0.1072(0.0422)^{*}$ \\
Education & $0.0894(0.0084)^{* * *}$ & $0.0918(0.0088)^{* * *}$ & $0.1561(0.0076)^{* * *}$ & $0.0943(0.0082)^{* * *}$ \\
APOE e2 & $-0.0247(0.0658)$ & $0.0107(0.0691)$ & $0.0608(0.0596)$ & $-0.0461(0.0642)$ \\
APOE e4 & $0.0345(0.0503)$ & $0.0198(0.0529)$ & $0.0237(0.0458)$ & $-0.0146(0.0493)$ \\
CTNNBL1 & $-0.0540(0.0668)$ & $-0.0175(0.0701)$ & $0.0237(0.0607)$ & $-0.0770(0.0653)$ \\
Rate of Change & & & & $-0.0396(0.0117)^{* * *}$ \\
Time & $-0.0154(0.0158)$ & $-0.0196(0.0154)$ & $0.0162(0.0091)$ & $0.0046(0.0040)$ \\
Gender & $0.0035(0.0054)$ & $-0.0046(0.0052)$ & $0.0019(0.0031)$ & $-0.0001(0.0008)$ \\
Education & $-0.0010(0.0010)$ & $0.0013(0.0010)$ & $-0.0000(0.0006)$ & $0.0054(0.0060)$ \\
APOE e2 & $-0.0017(0.0082)$ & $0.0157(0.0080)^{*}$ & $-0.0021(0.0046)$ & $-0.0053(0.0047)$ \\
APOE e4 & $-0.0184(0.0063)^{* *}$ & $0.0099(0.0061)$ & $-0.0017(0.0036)$ & $0.0061(0.0061)$ \\
CTNNBL1 & $0.0176(0.0083)^{*}$ & $0.0103(0.0081)$ & $-0.0021(0.0047)$ & 1686 \\
Individuals & 1687 & 1687 & 1682 & 0.5872 \\
Variance: Intercept & 0.4847 & 0.5826 & 0.5525 & 0.0008 \\
Variance: Time & 0.0012 & 0.0011 & 0.0008 & 0.1708 \\
Variance: Residual & 0.3379 & 0.3112 & 0.0891 & -4659.1763 \\
Log Likelihood & -5627.8502 & -5565.1423 & -3542.1389 & 0.90 \\
F-test & 2.27 & 0.89 & 0.13 & 0.092 \\
$R_{m}^{2}$ & 0.114 & 0.073 & 0.211 & 0.798 \\
$R_{c}^{2}$ & 0.63 & 0.667 & 0.885 & \\
\hline$F$-t & & & &
\end{tabular}

$F$-test with Kenward-Roger approximation

$R_{m}^{2}$ : Marginal $R^{2}$ statistics; $R_{c}^{2}$ : Conditional $R^{2}$ statistic

${ }^{* * *} p<0.001,{ }^{* *} p<0.01,{ }^{*} p<0.05$ 
Table 32: FRMD4A (1)

\begin{tabular}{|c|c|c|c|c|}
\hline & Episodic Memory & Digits Backwards & Spot-the-Word & Symbol Digits Modalities Test \\
\hline \multicolumn{5}{|l|}{ Initial Status } \\
\hline Gender & $0.4761(0.0433)^{* * *}$ & $-0.0550(0.0457)$ & $0.0112(0.0394)$ & $0.1130(0.0424)^{* *}$ \\
\hline$A P O E \mathrm{e} 2$ & $-0.0320(0.0661)$ & $-0.0017(0.0697)$ & $0.0644(0.0599)$ & $-0.0438(0.0644)$ \\
\hline$A P O E$ e 4 & $0.0378(0.0503)$ & $0.0143(0.0531)$ & $0.0280(0.0459)$ & $-0.0112(0.0493)$ \\
\hline FRMD4A_1 & $-0.0223(0.0429)$ & $0.0396(0.0453)$ & $0.0370(0.0391)$ & $-0.0065(0.0420)$ \\
\hline Gender & $0.0034(0.0054)$ & $-0.0054(0.0053)$ & $0.0015(0.0031)$ & $0.0048(0.0040)$ \\
\hline Education & $-0.0011(0.0010)$ & $0.0013(0.0010)$ & $-0.0000(0.0006)$ & $-0.0001(0.0008)$ \\
\hline$A P O E \mathrm{e} 2$ & $-0.0023(0.0082)$ & $0.0150(0.0080)$ & $-0.0021(0.0047)$ & $0.0052(0.0061)$ \\
\hline$A P O E \mathrm{e} 4$ & $-0.0194(0.0063)^{* *}$ & $0.0093(0.0061)$ & $-0.0024(0.0036)$ & $-0.0052(0.0047)$ \\
\hline FRMD4A_1 & $0.0066(0.0054)$ & $-0.0103(0.0052)^{*}$ & $-0.0048(0.0030)$ & $0.0011(0.0040)$ \\
\hline Log Likelihood & -5595.0460 & -5534.3969 & -3521.7654 & -4629.2447 \\
\hline$F$-test & 0.75 & 1.96 & 1.33 & 0.04 \\
\hline$R_{m}^{2}$ & 0.114 & 0.072 & 0.211 & 0.092 \\
\hline$R_{c}^{2}$ & 0.628 & 0.669 & 0.885 & 0.798 \\
\hline
\end{tabular}

$F$-test with Kenward-Roger approximation

$R_{m}^{2}$ : Marginal $R^{2}$ statistics; $R_{c}^{2}$ : Conditional $R^{2}$ statistic

${ }^{* * *} p<0.001,{ }^{* *} p<0.01,{ }^{*} p<0.05$ 
Table 33: FRMD4A (2)

\begin{tabular}{|c|c|c|c|c|}
\hline & Episodic Memory & Digits Backwards & Spot-the-Word & Symbol Digits Modalities Test \\
\hline Initial Status & & & & \\
\hline Intercept & $-1.3894(0.1262)^{* * *}$ & $-1.1436(0.1323)^{* * *}$ & $-2.0971(0.1148)^{* * *}$ & $-1.1988(0.1228)^{* * *}$ \\
\hline Gender & $0.4762(0.0434)^{* * *}$ & $-0.0488(0.0455)$ & $0.0059(0.0393)$ & $0.1127(0.0423)^{* *}$ \\
\hline Education & $0.0905(0.0084)^{* * *}$ & $0.0911(0.0088)^{* * *}$ & $0.1571(0.0077)^{* * *}$ & $0.0951(0.0082)^{* * *}$ \\
\hline$A P O E \mathrm{e} 2$ & $-0.0181(0.0659)$ & $0.0130(0.0691)$ & $0.0593(0.0595)$ & $-0.0384(0.0640)$ \\
\hline$A P O E$ e 4 & $0.0384(0.0505)$ & $0.0165(0.0529)$ & $0.0218(0.0458)$ & $-0.0043(0.0492)$ \\
\hline FRMD4A_2 & $-0.0014(0.0637)$ & $-0.1417(0.0668)^{*}$ & $0.1348(0.0578)^{*}$ & $-0.1019(0.0620)$ \\
\hline Rate of Change & & & & \\
\hline Time & $-0.0093(0.0158)$ & $-0.0228(0.0154)$ & $0.0177(0.0091)$ & $-0.0384(0.0117)^{* *}$ \\
\hline Gender & $0.0035(0.0054)$ & $-0.0055(0.0052)$ & $0.0023(0.0031)$ & $0.0045(0.0040)$ \\
\hline Education & $-0.0012(0.0011)$ & $0.0015(0.0010)$ & $-0.0001(0.0006)$ & $-0.0002(0.0008)$ \\
\hline$A P O E \mathrm{e} 2$ & $-0.0030(0.0082)$ & $0.0143(0.0079)$ & $-0.0018(0.0047)$ & $0.0046(0.0060)$ \\
\hline$A P O E \mathrm{e} 4$ & $-0.0199(0.0063)^{* *}$ & $0.0096(0.0061)$ & $-0.0019(0.0036)$ & $-0.0064(0.0047)$ \\
\hline FRMD4A_2 & $-0.0032(0.0080)$ & $0.0215(0.0077)^{* *}$ & $-0.0097(0.0045)^{*}$ & $0.0014(0.0059)$ \\
\hline Individuals & 1681 & 1681 & 1676 & 1680 \\
\hline Variance: Intercept & 0.4861 & 0.5809 & 0.5504 & 0.5838 \\
\hline Variance: Time & 0.0012 & 0.0010 & 0.0008 & 0.0007 \\
\hline Variance: Residual & 0.3378 & 0.3128 & 0.0892 & 0.1710 \\
\hline Log Likelihood & -5610.6513 & -5548.6451 & -3530.1780 & -4638.1688 \\
\hline$F$-test & 0.12 & $4.29^{*}$ & $3.63^{*}$ & 1.39 \\
\hline$R_{m}^{2}$ & 0.114 & 0.073 & 0.213 & 0.093 \\
\hline$R_{c}^{2}$ & 0.63 & 0.666 & 0.885 & 0.798 \\
\hline
\end{tabular}

$F$-test with Kenward-Roger approximation

$R_{m}^{2}$ : Marginal $R^{2}$ statistics; $R_{c}^{2}$ : Conditional $R^{2}$ statistic

${ }^{* * *} p<0.001,{ }^{* *} p<0.01,{ }^{*} p<0.05$ 
Table 34: Intergenic ChrX

\begin{tabular}{|c|c|c|c|c|}
\hline \multirow{2}{*}{$\begin{array}{c}\text { Initial Status } \\
\text { Intercept }\end{array}$} & Episodic Memory & Digits Backwards & Spot-the-Word & Symbol Digits Modalities Test \\
\hline & & & & \\
\hline Gender & $0.4785(0.0434)^{* * *}$ & $-0.0430(0.0456)$ & $0.0069(0.0396)$ & $0.1068(0.0426)^{*}$ \\
\hline$A P O E \mathrm{e} 2$ & $-0.0260(0.0657)$ & $0.0135(0.0690)$ & $0.0576(0.0596)$ & $-0.0440(0.0643)$ \\
\hline$A P O E \mathrm{e} 4$ & $0.0311(0.0502)$ & $0.0175(0.0528)$ & $0.0197(0.0459)$ & $-0.0090(0.0494)$ \\
\hline inter_x & $0.0310(0.0745)$ & $-0.1325(0.0785)$ & $-0.0547(0.0679)$ & $0.0160(0.0733)$ \\
\hline Gender & $0.0038(0.0054)$ & $-0.0052(0.0053)$ & $0.0018(0.0031)$ & $0.0040(0.0040)$ \\
\hline Education & $-0.0009(0.0010)$ & $0.0012(0.0010)$ & $-0.0000(0.0006)$ & $-0.0001(0.0008)$ \\
\hline$A P O E \mathrm{e} 2$ & $-0.0030(0.0081)$ & $0.0145(0.0079)$ & $-0.0016(0.0047)$ & $0.0051(0.0060)$ \\
\hline$A P O E \mathrm{e} 4$ & $-0.0192(0.0063)^{* *}$ & $0.0097(0.0061)$ & $-0.0018(0.0036)$ & $-0.0059(0.0047)$ \\
\hline inter_x & $-0.0232(0.0092)^{*}$ & $0.0018(0.0090)$ & $0.0040(0.0053)$ & $0.0024(0.0068)$ \\
\hline Log Likelihood & -5586.0617 & -5525.8787 & -3524.9632 & -4633.4901 \\
\hline$F$-test & $3.56^{*}$ & 1.64 & 0.44 & 0.13 \\
\hline$R_{m}^{2}$ & 0.115 & 0.073 & 0.21 & 0.091 \\
\hline$R_{c}^{2}$ & 0.627 & 0.667 & 0.884 & 0.798 \\
\hline
\end{tabular}

$F$-test with Kenward-Roger approximation

$R_{m}^{2}$ : Marginal $R^{2}$ statistics; $R_{c}^{2}$ : Conditional $R^{2}$ statistic

${ }^{* * *} p<0.001,{ }^{* *} p<0.01,{ }^{*} p<0.05$ 
Table 35: LGALS3

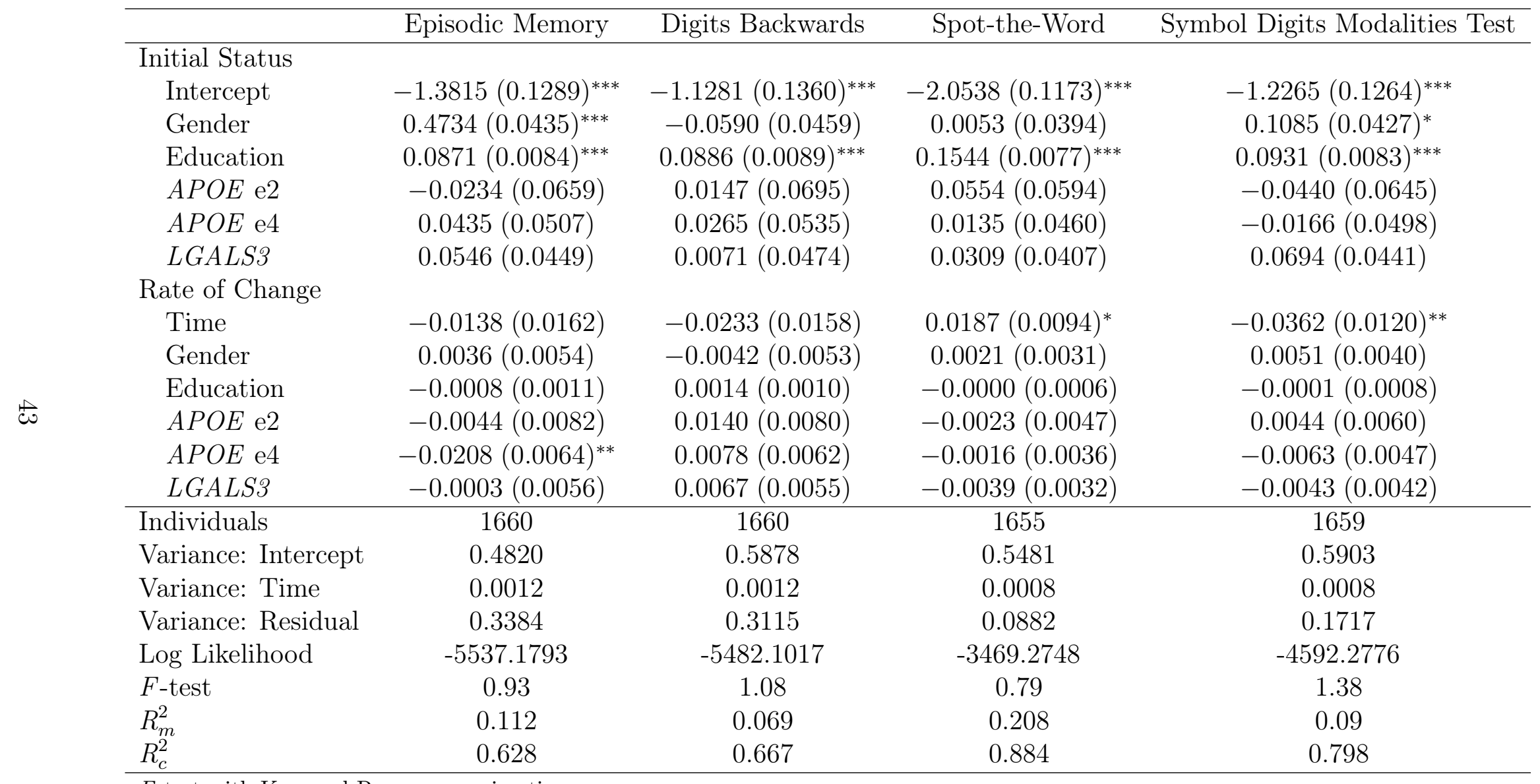

$F$-test with Kenward-Roger approximation

$R_{m}^{2}$ : Marginal $R^{2}$ statistics; $R_{c}^{2}$ : Conditional $R^{2}$ statistic

${ }^{* * *} p<0.001,{ }^{* *} p<0.01,{ }^{*} p<0.05$ 
Table 36: MMP12

\begin{tabular}{|c|c|c|c|c|}
\hline & Episodic Memory & Digits Backwards & Spot-the-Word & Symbol Digits Modalities Test \\
\hline \multicolumn{5}{|l|}{ Initial Status } \\
\hline Gender & $0.4739(0.0433)^{* * *}$ & $-0.0543(0.0454)$ & $0.0085(0.0393)$ & $0.1087(0.0422)^{*}$ \\
\hline Education & $0.0891(0.0084)^{* * *}$ & $0.0911(0.0088)^{* * *}$ & $0.1560(0.0076)^{* * *}$ & $0.0948(0.0082)^{* * *}$ \\
\hline APOE e2 & $-0.0210(0.0660)$ & $-0.0005(0.0692)$ & $0.0559(0.0597)$ & $-0.0477(0.0642)$ \\
\hline APOE e4 & $0.0365(0.0503)$ & $0.0128(0.0528)$ & $0.0196(0.0458)$ & $-0.0135(0.0491)$ \\
\hline MMP12 & $0.0020(0.0479)$ & $0.0116(0.0501)$ & $0.0067(0.0434)$ & $0.0632(0.0466)$ \\
\hline Rate of Change & & & & \\
\hline Time & $-0.0096(0.0159)$ & $-0.0146(0.0155)$ & $0.0167(0.0092)$ & $-0.0378(0.0117)^{* *}$ \\
\hline Gender & $0.0033(0.0054)$ & $-0.0047(0.0052)$ & $0.0020(0.0031)$ & $0.0046(0.0040)$ \\
\hline Education & $-0.0011(0.0010)$ & $0.0013(0.0010)$ & $-0.0001(0.0006)$ & $-0.0001(0.0008)$ \\
\hline APOE e2 & $-0.0044(0.0082)$ & $0.0143(0.0080)$ & $-0.0021(0.0047)$ & $0.0051(0.0061)$ \\
\hline APOE e4 & $-0.0194(0.0063)^{* *}$ & $0.0096(0.0061)$ & $-0.0017(0.0036)$ & $-0.0057(0.0047)$ \\
\hline MMP12 & $-0.0061(0.0059)$ & $-0.0095(0.0058)$ & $-0.0007(0.0034)$ & $-0.0035(0.0044)$ \\
\hline Individuals & 1684 & 1684 & 1679 & 1683 \\
\hline Variance: Intercept & 0.4865 & 0.5815 & 0.5532 & 0.5850 \\
\hline Variance: Time & 0.0012 & 0.0011 & 0.0008 & 0.0008 \\
\hline Variance: Residual & 0.3372 & 0.3113 & 0.0888 & 0.1699 \\
\hline Log Likelihood & -5616.3168 & -5554.7791 & -3532.8400 & -4644.0230 \\
\hline$F$-test & 0.67 & 1.55 & 0.03 & 0.98 \\
\hline$R_{m}^{2}$ & 0.113 & 0.072 & 0.21 & 0.092 \\
\hline$R_{c}^{2}$ & 0.63 & 0.667 & 0.885 & 0.799 \\
\hline
\end{tabular}

$F$-test with Kenward-Roger approximation

$R_{m}^{2}$ : Marginal $R^{2}$ statistics; $R_{c}^{2}$ : Conditional $R^{2}$ statistic

${ }^{* * *} p<0.001,{ }^{* *} p<0.01,{ }^{*} p<0.05$ 
Table 37: MTHFD1L

\begin{tabular}{lcccc}
\hline & Episodic Memory & Digits Backwards & Spot-the-Word & Symbol Digits Modalities Test \\
\hline Initial Status & & & & \\
Intercept & $-1.3803(0.1255)^{* * *}$ & $-1.1555(0.1321)^{* * *}$ & $-2.0649(0.1146)^{* * *}$ & $-1.2129(0.1227)^{* * *}$ \\
Gender & $0.4761(0.0432)^{* * *}$ & $-0.0578(0.0454)$ & $0.0070(0.0392)$ & $0.1073(0.0422)^{*}$ \\
Education & $0.0895(0.0084)^{* * *}$ & $0.0912(0.0088)^{* * *}$ & $0.1558(0.0076)^{* * *}$ & $0.0948(0.0082)^{* * *}$ \\
APOE e2 & $-0.0209(0.0657)$ & $0.0121(0.0691)$ & $0.0602(0.0595)$ & $-0.0410(0.0641)$ \\
APOE e4 & $0.0361(0.0502)$ & $0.0174(0.0528)$ & $0.0229(0.0457)$ & $-0.0100(0.0492)$ \\
MTHFD1L & $0.0249(0.0583)$ & $-0.0431(0.0613)$ & $0.0136(0.0529)$ & $0.0332(0.0570)$ \\
Rate of Change & & & & $-0.0371(0.0116)^{* *}$ \\
Time & $-0.0115(0.0157)$ & $-0.0172(0.0154)$ & $0.0171(0.0091)$ & $0.0042(0.0040)$ \\
Gender & $0.0031(0.0054)$ & $-0.0045(0.0052)$ & $0.0020(0.0031)$ & $-0.0002(0.0008)$ \\
Education & $-0.0010(0.0010)$ & $0.0013(0.0010)$ & $-0.0000(0.0006)$ & $0.0052(0.0060)$ \\
APOE e2 & $-0.0029(0.0081)$ & $0.0147(0.0079)$ & $-0.0019(0.0046)$ & $-0.0054(0.0046)$ \\
APOE e4 & $-0.0192(0.0063)^{* *}$ & $0.0094(0.0061)$ & $-0.0018(0.0036)$ & $-0.0053(0.0054)$ \\
MTHFD1L & $-0.0006(0.0073)$ & $-0.0016(0.0070)$ & $-0.0046(0.0041)$ & 1685 \\
Individuals & 1686 & 1686 & 1681 & 0.5869 \\
Variance: Intercept & 0.4828 & 0.5840 & 0.5524 & 0.0007 \\
Variance: Time & 0.0011 & 0.0011 & 0.0008 & 0.1710 \\
Variance: Residual & 0.3379 & 0.3116 & 0.0892 & -4653.5998 \\
Log Likelihood & -5621.4661 & -5565.9494 & -3541.6760 & 0.53 \\
$F$-test & 0.10 & 0.44 & 0.63 & 0.092 \\
$R_{m}^{2}$ & 0.629 & 0.072 & 0.21 & 0.798 \\
$R_{c}^{2}$ & 0.668 & 0.885 & \\
\hline$F$-tit & & & &
\end{tabular}

$F$-test with Kenward-Roger approximation

$R_{m}^{2}$ : Marginal $R^{2}$ statistics; $R_{c}^{2}$ : Conditional $R^{2}$ statistic

${ }^{* * *} p<0.001,{ }^{* *} p<0.01,{ }^{*} p<0.05$ 
Table 38: PAICS

\begin{tabular}{lcccc}
\hline & Episodic Memory & Digits Backwards & Spot-the-Word & Symbol Digits Modalities Test \\
\hline Initial Status & & & & \\
Intercept & $-1.3733(0.1253)^{* * *}$ & $-1.1482(0.1319)^{* * *}$ & $-2.0499(0.1142)^{* * *}$ & $-1.1990(0.1224)^{* * *}$ \\
Gender & $0.4777(0.0432)^{* * *}$ & $-0.0567(0.0455)$ & $0.0052(0.0392)$ & $0.1113(0.0423)^{* *}$ \\
Education & $0.0891(0.0084)^{* * *}$ & $0.0910(0.0088)^{* * *}$ & $0.1556(0.0076)^{* * *}$ & $0.0949(0.0082)^{* * *}$ \\
APOE e2 & $-0.0203(0.0658)$ & $0.0065(0.0692)$ & $0.0569(0.0595)$ & $-0.0460(0.0642)$ \\
APOE e4 & $0.0378(0.0503)$ & $0.0167(0.0529)$ & $0.0255(0.0457)$ & $-0.0131(0.0493)$ \\
PAICS & $0.0289(0.0655)$ & $-0.0777(0.0690)$ & $-0.0698(0.0596)$ & $-0.0840(0.0642)$ \\
Rate of Change & & & & $-0.0375(0.0116)^{* *}$ \\
Time & $-0.0099(0.0157)$ & $-0.0180(0.0153)$ & $0.0158(0.0091)$ & $0.0045(0.0040)$ \\
Gender & $0.0030(0.0054)$ & $-0.0049(0.0052)$ & $0.0020(0.0031)$ & $-0.0002(0.0008)$ \\
Education & $-0.0011(0.0010)$ & $0.0013(0.0010)$ & $-0.0000(0.0006)$ & $0.0049(0.0060)$ \\
APOE e2 & $-0.0034(0.0082)$ & $0.0152(0.0080)$ & $-0.0018(0.0047)$ & $-0.0059(0.0047)$ \\
APOE e4 & $-0.0194(0.0063)^{* *}$ & $0.0095(0.0061)$ & $-0.0017(0.0036)$ & $0.0004(0.0062)$ \\
PAICS & $-0.0087(0.0083)$ & $0.0060(0.0081)$ & $0.0036(0.0047)$ & 1684 \\
Individuals & 1685 & 1685 & 1680 & 0.5873 \\
Variance: Intercept & 0.4829 & 0.5861 & 0.5510 & 0.0008 \\
Variance: Time & 0.0011 & 0.0012 & 0.0008 & 0.1709 \\
Variance: Residual & 0.3385 & 0.3102 & 0.0886 & -4652.2568 \\
Log Likelihood & -5619.0860 & -5559.5847 & -3531.2662 & 0.93 \\
$F$-test & 0.54 & 0.66 & 0.75 & 0.092 \\
$R_{m}^{2}$ & 0.113 & 0.072 & 0.211 & 0.798 \\
$R_{c}^{2}$ & 0.628 & 0.669 & 0.885 & \\
\hline$F$-t & & & &
\end{tabular}

$F$-test with Kenward-Roger approximation

$R_{m}^{2}$ : Marginal $R^{2}$ statistics; $R_{c}^{2}$ : Conditional $R^{2}$ statistic

${ }^{* * *} p<0.001,{ }^{* *} p<0.01,{ }^{*} p<0.05$ 
Table 39: $P D E E^{r} A$

\begin{tabular}{lcccc}
\hline & Episodic Memory & Digits Backwards & Spot-the-Word & Symbol Digits Modalities Test \\
\hline Initial Status & & & & \\
Intercept & $-1.3419(0.1300)^{* * *}$ & $-1.0930(0.1358)^{* * *}$ & $-2.0376(0.1179)^{* * *}$ & $-1.1544(0.1264)^{* * *}$ \\
Gender & $0.4720(0.0435)^{* * *}$ & $-0.0553(0.0455)$ & $-0.0009(0.0393)$ & $0.0998(0.0424)^{*}$ \\
Education & $0.0893(0.0084)^{* * *}$ & $0.0913(0.0088)^{* * *}$ & $0.1562(0.0076)^{* * *}$ & $0.0947(0.0082)^{* * *}$ \\
APOE e2 & $-0.0143(0.0663)$ & $0.0131(0.0692)$ & $0.0608(0.0596)$ & $-0.0325(0.0643)$ \\
APOE e4 & $0.0358(0.0506)$ & $0.0102(0.0528)$ & $0.0206(0.0458)$ & $-0.0132(0.0493)$ \\
PDE7A & $-0.0393(0.0460)$ & $-0.1028(0.0481)^{*}$ & $-0.0320(0.0416)$ & $-0.0771(0.0449)$ \\
Rate of Change & & & & $-0.0421(0.0120)^{* * *}$ \\
Time & $-0.0147(0.0163)$ & $-0.0236(0.0159)$ & $0.0160(0.0094)$ & $0.0044(0.0040)$ \\
Gender & $0.0030(0.0054)$ & $-0.0041(0.0053)$ & $0.0019(0.0031)$ & $-0.0003(0.0008)$ \\
Education & $-0.0011(0.0011)$ & $0.0012(0.0010)$ & $-0.0000(0.0006)$ & $0.0039(0.0060)$ \\
APOE e2 & $-0.0030(0.0082)$ & $0.0139(0.0080)$ & $-0.0025(0.0047)$ & $-0.0058(0.0047)$ \\
APOE e4 & $-0.0206(0.0063)^{* *}$ & $0.0105(0.0062)$ & $-0.0023(0.0036)$ & $0.0089(0.0042)^{*}$ \\
PDE7A & $0.0065(0.0058)$ & $0.0096(0.0056)$ & $-0.0001(0.0033)$ & 1663 \\
Individuals & 1664 & 1664 & 1659 & 0.5816 \\
Variance: Intercept & 0.4846 & 0.5766 & 0.5470 & 0.0007 \\
Variance: Time & 0.0011 & 0.0012 & 0.0008 & 0.1712 \\
Variance: Residual & 0.3396 & 0.3098 & 0.0893 & -4584.1715 \\
Log Likelihood & -5551.9141 & -5480.8181 & -3490.5474 & 2.76 \\
$F$-test & 0.69 & 2.60 & 0.35 & 0.092 \\
$R_{m}^{2}$ & 0.113 & 0.073 & 0.213 & 0.796 \\
$R_{c}^{2}$ & 0.627 & 0.668 & 0.884 &
\end{tabular}

$F$-test with Kenward-Roger approximation

$R_{m}^{2}$ : Marginal $R^{2}$ statistics; $R_{c}^{2}$ : Conditional $R^{2}$ statistic

${ }^{* * *} p<0.001,{ }^{* *} p<0.01,{ }^{*} p<0.05$ 
Table 40: SNTG1

\begin{tabular}{lcccc}
\hline & Episodic Memory & Digits Backwards & Spot-the-Word & Symbol Digits Modalities Test \\
\hline Initial Status & & & & \\
Intercept & $-1.4248(0.1281)^{* * *}$ & $-1.1881(0.1348)^{* * *}$ & $-2.0312(0.1166)^{* * *}$ & $-1.2098(0.1251)^{* * *}$ \\
Gender & $0.4757(0.0432)^{* * *}$ & $-0.0531(0.0455)$ & $0.0112(0.0391)$ & $0.1103(0.0422)^{* *}$ \\
Education & $0.0888(0.0084)^{* * *}$ & $0.0912(0.0088)^{* * *}$ & $0.1560(0.0076)^{* * *}$ & $0.0946(0.0082)^{* * *}$ \\
APOE e2 & $-0.0210(0.0658)$ & $0.0052(0.0693)$ & $0.0623(0.0594)$ & $-0.0417(0.0641)$ \\
APOE e4 & $0.0371(0.0502)$ & $0.0155(0.0528)$ & $0.0196(0.0455)$ & $-0.0126(0.0491)$ \\
SNTG1 & $0.0864(0.0460)$ & $0.0378(0.0484)$ & $-0.0480(0.0417)$ & $0.0079(0.0450)$ \\
Rate of Change & & & & $-0.0375(0.0119)^{* *}$ \\
Time & $-0.0068(0.0161)$ & $-0.0158(0.0157)$ & $0.0158(0.0093)$ & $0.0045(0.0040)$ \\
Gender & $0.0032(0.0054)$ & $-0.0051(0.0052)$ & $0.0020(0.0031)$ & $-0.0001(0.0008)$ \\
Education & $-0.0010(0.0010)$ & $0.0013(0.0010)$ & $-0.0000(0.0006)$ & $0.0047(0.0060)$ \\
APOE e2 & $-0.0028(0.0082)$ & $0.0158(0.0079)^{*}$ & $-0.0024(0.0046)$ & $-0.0056(0.0047)$ \\
APOE e4 & $-0.0195(0.0063)^{* *}$ & $0.0094(0.0061)$ & $-0.0018(0.0036)$ & $-0.0015(0.0042)$ \\
SNTG1 & $-0.0084(0.0057)$ & $-0.0025(0.0056)$ & $0.0002(0.0033)$ & 1685 \\
Individuals & 1686 & 1686 & 1681 & 0.5858 \\
Variance: Intercept & 0.4832 & 0.5842 & 0.5478 & 0.0008 \\
Variance: Time & 0.0012 & 0.0011 & 0.0008 & 0.1710 \\
Variance: Residual & 0.3376 & 0.3117 & 0.0891 & -4656.2925 \\
Log Likelihood & -5622.8872 & -5565.5717 & -3534.8577 & 0.06 \\
F-test & 1.94 & 0.31 & 0.74 & 0.092 \\
$R_{m}^{2}$ & 0.115 & 0.072 & 0.212 & 0.798 \\
$R_{c}^{2}$ & 0.63 & 0.667 & 0.884 & \\
\hline$F$-t & & & &
\end{tabular}

$F$-test with Kenward-Roger approximation

$R_{m}^{2}$ : Marginal $R^{2}$ statistics; $R_{c}^{2}$ : Conditional $R^{2}$ statistic

${ }^{* * *} p<0.001,{ }^{* *} p<0.01,{ }^{*} p<0.05$ 
Table 41: SORL1

\begin{tabular}{lcccc}
\hline & Episodic Memory & Digits Backwards & Spot-the-Word & Symbol Digits Modalities Test \\
\hline Initial Status & & & & \\
Intercept & $-1.3745(0.1299)^{* * *}$ & $-1.1530(0.1363)^{* * *}$ & $-2.1243(0.1184)^{* * *}$ & $-1.2185(0.1267)^{* * *}$ \\
Gender & $0.4770(0.0435)^{* * *}$ & $-0.0509(0.0457)$ & $0.0067(0.0395)$ & $0.1129(0.0425)^{* *}$ \\
Education & $0.0873(0.0084)^{* * *}$ & $0.0900(0.0088)^{* * *}$ & $0.1559(0.0077)^{* * *}$ & $0.0951(0.0082)^{* * *}$ \\
APOE e2 & $-0.0406(0.0666)$ & $-0.0087(0.0698)$ & $0.0613(0.0602)$ & $-0.0436(0.0648)$ \\
APOE e4 & $0.0303(0.0507)$ & $0.0240(0.0532)$ & $0.0228(0.0461)$ & $-0.0165(0.0496)$ \\
SORL1 & $0.0461(0.0468)$ & $0.0056(0.0492)$ & $0.0836(0.0426)^{*}$ & $0.0103(0.0458)$ \\
Rate of Change & & & & $-0.0407(0.0120)^{* * *}$ \\
Time & $-0.0104(0.0163)$ & $-0.0226(0.0158)$ & $0.0210(0.0094)^{*}$ & $0.0049(0.0040)$ \\
Gender & $0.0023(0.0054)$ & $-0.0056(0.0053)$ & $0.0023(0.0031)$ & $-0.0002(0.0008)$ \\
Education & $-0.0007(0.0011)$ & $0.0013(0.0010)$ & $-0.0001(0.0006)$ & $0.0037(0.0061)$ \\
APOE e2 & $-0.0018(0.0083)$ & $0.0149(0.0080)$ & $-0.0031(0.0047)$ & $-0.0059(0.0047)$ \\
APOE e4 & $-0.0190(0.0064)^{* *}$ & $0.0095(0.0062)$ & $-0.0018(0.0036)$ & $0.0051(0.0043)$ \\
SORL1 & $-0.0072(0.0059)$ & $0.0065(0.0057)$ & $-0.0064(0.0033)$ & 1663 \\
Individuals & 1664 & 1664 & 1659 & 0.5863 \\
Variance: Intercept & 0.4856 & 0.5803 & 0.5528 & 0.0007 \\
Variance: Time & 0.0012 & 0.0010 & 0.0008 & 0.1723 \\
Variance: Residual & 0.3378 & 0.3138 & 0.0895 & -4592.4832 \\
Log Likelihood & -5550.1301 & -5491.1402 & -3503.0898 & 0.92 \\
$F$-test & 0.85 & 0.94 & 2.73 & 0.092 \\
$R_{m}^{2}$ & 0.113 & 0.071 & 0.21 & 0.796 \\
$R_{c}^{2}$ & 0.63 & 0.663 & 0.884 &
\end{tabular}

$F$-test with Kenward-Roger approximation

$R_{m}^{2}$ : Marginal $R^{2}$ statistics; $R_{c}^{2}$ : Conditional $R^{2}$ statistic

${ }^{* * *} p<0.001,{ }^{* *} p<0.01,{ }^{*} p<0.05$ 
Table 42: SPON1

\begin{tabular}{lcccc}
\hline & Episodic Memory & Digits Backwards & Spot-the-Word & Symbol Digits Modalities Test \\
\hline Initial Status & & & & \\
Intercept & $-1.3728(0.1252)^{* * *}$ & $-1.1560(0.1316)^{* * *}$ & $-2.0536(0.1140)^{* * *}$ & $-1.2059(0.1223)^{* * *}$ \\
Gender & $0.4776(0.0432)^{* * *}$ & $-0.0547(0.0454)$ & $0.0068(0.0392)$ & $0.1078(0.0422)^{*}$ \\
Education & $0.0890(0.0084)^{* * *}$ & $0.0914(0.0088)^{* * *}$ & $0.1560(0.0076)^{* * *}$ & $0.0946(0.0082)^{* * *}$ \\
APOE e2 & $-0.0246(0.0658)$ & $0.0121(0.0691)$ & $0.0611(0.0594)$ & $-0.0420(0.0641)$ \\
APOE e4 & $0.0310(0.0503)$ & $0.0164(0.0529)$ & $0.0229(0.0457)$ & $-0.0102(0.0493)$ \\
SPON1 & $0.0585(0.0687)$ & $-0.0930(0.0722)$ & $-0.1037(0.0626)$ & $0.0093(0.0673)$ \\
Rate of Change & & & & $-0.0380(0.0116)^{* *}$ \\
Time & $-0.0116(0.0157)$ & $-0.0180(0.0153)$ & $0.0155(0.0091)$ & $0.0046(0.0040)$ \\
Gender & $0.0032(0.0054)$ & $-0.0048(0.0052)$ & $0.0020(0.0031)$ & $-0.0002(0.0008)$ \\
Education & $-0.0010(0.0010)$ & $0.0013(0.0010)$ & $-0.0000(0.0006)$ & $0.0051(0.0060)$ \\
APOE e2 & $-0.0028(0.0082)$ & $0.0147(0.0079)$ & $-0.0020(0.0046)$ & $-0.0058(0.0047)$ \\
APOE e4 & $-0.0190(0.0063)^{* *}$ & $0.0094(0.0061)$ & $-0.0021(0.0036)$ & $-0.0037(0.0064)$ \\
SPON1 & $-0.0091(0.0087)$ & $0.0075(0.0084)$ & $0.0067(0.0050)$ & 1686 \\
Individuals & 1687 & 1687 & 1682 & 0.5876 \\
Variance: Intercept & 0.4846 & 0.5843 & 0.5513 & 0.0008 \\
Variance: Time & 0.0012 & 0.0011 & 0.0008 & 0.1710 \\
Variance: Residual & 0.3377 & 0.3114 & 0.0890 & -4659.6870 \\
Log Likelihood & -5627.9148 & -5568.9914 & -3541.2258 & 0.17 \\
F-test & 0.62 & 0.88 & 1.69 & 0.091 \\
$R_{m}^{2}$ & 0.114 & 0.072 & 0.211 & 0.798 \\
$R_{c}^{2}$ & 0.63 & 0.668 & 0.885 & \\
\hline$F$-t & & & &
\end{tabular}

$F$-test with Kenward-Roger approximation

$R_{m}^{2}$ : Marginal $R^{2}$ statistics; $R_{c}^{2}$ : Conditional $R^{2}$ statistic

${ }^{* * *} p<0.001,{ }^{* *} p<0.01,{ }^{*} p<0.05$ 
Table 43: ZNF224

\begin{tabular}{lcccc}
\hline & Episodic Memory & Digits Backwards & Spot-the-Word & Symbol Digits Modalities Test \\
\hline Initial Status & & & & $-1.2192(0.1230)^{* * *}$ \\
Intercept & $-1.3761(0.1260)^{* * *}$ & $-1.1897(0.1323)^{* * *}$ & $-2.0947(0.1147)^{* * *}$ & $0.1101(0.0422)^{* *}$ \\
Gender & $0.4755(0.0432)^{* * *}$ & $-0.0547(0.0454)$ & $0.0080(0.0392)$ & $0.0946(0.0082)^{* * *}$ \\
Education & $0.0894(0.0084)^{* * *}$ & $0.0917(0.0088)^{* * *}$ & $0.1561(0.0076)^{* * *}$ & $-0.0436(0.0641)$ \\
APOE e2 & $-0.0229(0.0658)$ & $0.0074(0.0690)$ & $0.0552(0.0594)$ & $-0.0140(0.0493)$ \\
APOE e4 & $0.0364(0.0503)$ & $0.0156(0.0529)$ & $0.0192(0.0457)$ & $0.0468(0.0453)$ \\
ZNF224 & $0.0121(0.0464)$ & $0.0706(0.0487)$ & $0.1001(0.0420)^{*}$ & $-0.0398(0.0117)^{* * *}$ \\
Rate of Change & & & & $0.0045(0.0040)$ \\
Time & $-0.0117(0.0158)$ & $-0.0197(0.0154)$ & $0.0163(0.0091)$ & $-0.0002(0.0008)$ \\
Gender & $0.0033(0.0054)$ & $-0.0046(0.0052)$ & $0.0019(0.0031)$ & $0.0048(0.0060)$ \\
Education & $-0.0010(0.0010)$ & $0.0013(0.0010)$ & $-0.0000(0.0006)$ & $-0.0057(0.0047)$ \\
APOE e2 & $-0.0029(0.0082)$ & $0.0146(0.0079)$ & $-0.0019(0.0046)$ & $0.0050(0.0043)$ \\
APOE e4 & $-0.0193(0.0063)^{* *}$ & $0.0089(0.0061)$ & $-0.0016(0.0036)$ & 1686 \\
ZNF224 & $-0.0019(0.0058)$ & $0.0069(0.0056)$ & $-0.0009(0.0033)$ & 0.5871 \\
Individuals & 1687 & 1687 & 1682 & 0.0008 \\
Variance: Intercept & 0.4851 & 0.5828 & 0.5506 & 0.1709 \\
Variance: Time & 0.0012 & 0.0011 & 0.0008 & -4658.0289 \\
Variance: Residual & 0.3378 & 0.3112 & 0.0891 & 1.81 \\
Log Likelihood & -5629.1238 & -5562.5897 & -3539.3767 & 0.093 \\
F-test & 0.06 & $3.43^{*}$ & $3.04 *$ & 0.798 \\
$R_{m}^{2}$ & 0.114 & 0.075 & 0.213 & 0.885 \\
$R_{c}^{2}$ & 0.63 & 0.668 & &
\end{tabular}

$F$-test with Kenward-Roger approximation

$R_{m}^{2}$ : Marginal $R^{2}$ statistics; $R_{c}^{2}$ : Conditional $R^{2}$ statistic

${ }^{* * *} p<0.001,{ }^{* *} p<0.01,{ }^{*} p<0.05$ 
Table 44: Top LOAD risk SNPs and GRS: Parameter estimates and model fit statistics for SNP/GRS main effects according to last diagnosis

\begin{tabular}{|c|c|c|c|c|c|}
\hline & & Episodic & Digits & Spot-the-Word & Symbol Digits \\
\hline & & Memory & Backwards & & Modalities Test \\
\hline & & Estimate (SE) & Estimate (SE) & Estimate (SE) & Estimate (SE) \\
\hline \multirow[t]{3}{*}{$A P O E \in \mathscr{2}$} & Intercept & $-0.032(0.071)$ & $0.037(0.074)$ & $0.039(0.064)$ & $-0.028(0.069)$ \\
\hline & Slope - CN & $-0.002(0.009)$ & $0.016(0.009)$ & $0.000(0.005)$ & $0.002(0.006)$ \\
\hline & Slope - CI & $0.007(0.024)$ & $0.035(0.025)$ & $-0.017(0.015)$ & $0.021(0.020)$ \\
\hline \multirow[t]{4}{*}{$A P O E \in 4$} & Intercept & $0.044(0.054)$ & $0.011(0.057)$ & $0.028(0.049)$ & $-0.010(0.053)$ \\
\hline & Slope - CN & $-0.015(0.007)^{*}$ & $0.013(0.007)^{*}$ & $0.000(0.004)$ & $-0.007(0.005)$ \\
\hline & Slope - CI & $-0.050(0.020)^{*}$ & $0.004(0.021)$ & $-0.017(0.013)$ & $-0.002(0.016)$ \\
\hline & $F$-test & 1.93 & 1.96 & 0.50 & 0.67 \\
\hline \multirow[t]{4}{*}{$A B C A$ 7-rs3764650 } & Intercept & $-0.173(0.059)^{* *}$ & $0.021(0.063)$ & $-0.040(0.054)$ & $-0.053(0.058)$ \\
\hline & Slope - CN & $0.004(0.007)$ & $0.007(0.007)$ & $-0.000(0.004)$ & $0.008(0.005)$ \\
\hline & Slope - CI & $-0.026(0.020)$ & $-0.041(0.021)^{*}$ & $-0.004(0.013)$ & $-0.029(0.016)$ \\
\hline & $F$-test & $4.32^{* *}$ & 1.81 & 0.27 & 1.99 \\
\hline \multirow[t]{3}{*}{ BIN1-rs744373 } & Intercept & $-0.034(0.046)$ & $0.079(0.048)$ & $-0.015(0.041)$ & $0.043(0.044)$ \\
\hline & Slope - CN & $-0.008(0.006)$ & $-0.002(0.006)$ & $-0.003(0.003)$ & $-0.004(0.004)$ \\
\hline & Slope - CI & $0.016(0.017)$ & $0.003(0.017)$ & $0.014(0.011)$ & $0.024(0.014)$ \\
\hline
\end{tabular}




\begin{tabular}{|c|c|c|c|c|c|c|}
\hline & & $F$-test & 1.73 & 1.01 & 0.97 & 1.62 \\
\hline & CD2AP-rs9296559 & Intercept & $0.046(0.046)$ & $0.040(0.048)$ & $-0.010(0.042)$ & $0.011(0.045)$ \\
\hline & & Slope - CN & $-0.007(0.006)$ & $-0.001(0.006)$ & $-0.003(0.003)$ & $-0.000(0.004)$ \\
\hline & & Slope - CI & $-0.003(0.016)$ & $0.013(0.017)$ & $0.009(0.010)$ & $-0.002(0.013)$ \\
\hline & & $F$-test & 0.59 & 0.53 & 0.62 & 0.03 \\
\hline & CD33-rs34813869 & Intercept & $0.000(0.072)$ & $-0.056(0.076)$ & $-0.041(0.065)$ & $0.086(0.070)$ \\
\hline & & Slope - CN & $-0.000(0.009)$ & $-0.004(0.009)$ & $0.002(0.005)$ & $-0.006(0.007)$ \\
\hline & & Slope - CI & $-0.034(0.026)$ & $-0.033(0.026)$ & $-0.008(0.016)$ & $0.002(0.021)$ \\
\hline & & $F$-test & 0.55 & 1.09 & 0.25 & 0.57 \\
\hline 0 & $C L U-\mathrm{rs} 11136000$ & Intercept & $0.016(0.062)$ & $0.060(0.065)$ & $0.013(0.055)$ & $0.055(0.060)$ \\
\hline & & Slope - CN & $0.005(0.008)$ & $0.002(0.008)$ & $0.004(0.004)$ & $0.005(0.006)$ \\
\hline & & Slope - CI & $-0.012(0.021)$ & $-0.008(0.021)$ & $-0.012(0.013)$ & $-0.025(0.017)$ \\
\hline & & $F$-test & 0.37 & 0.54 & 0.74 & 1.52 \\
\hline & CR1-rs3818361 & Intercept & $-0.029(0.049)$ & $0.018(0.052)$ & $-0.054(0.044)$ & $-0.032(0.048)$ \\
\hline & & Slope - CN & $-0.010(0.006)$ & $-0.003(0.006)$ & $0.001(0.003)$ & $0.000(0.005)$ \\
\hline & & Slope - CI & $-0.011(0.016)$ & $-0.023(0.017)$ & $0.004(0.010)$ & $0.003(0.013)$ \\
\hline & & $F$-test & 2.07 & 0.72 & 0.50 & 0.17 \\
\hline & EPHA1-rs11767557 & Intercept & $0.071(0.128)$ & $0.113(0.135)$ & $-0.078(0.116)$ & $-0.267(0.125)^{*}$ \\
\hline & & Slope - CN & $-0.019(0.016)$ & $-0.026(0.015)$ & $0.004(0.009)$ & $0.005(0.012)$ \\
\hline
\end{tabular}




\begin{tabular}{|c|c|c|c|c|c|}
\hline & Slope - CI & $0.061(0.052)$ & $0.064(0.053)$ & $-0.021(0.032)$ & $0.086(0.043)^{*}$ \\
\hline & $F$-test & 1.07 & 1.55 & 0.35 & 2.60 \\
\hline$M S_{4} A_{4} A$-rs4938933 & Intercept & $-0.080(0.061)$ & $-0.043(0.064)$ & $0.093(0.055)$ & $0.058(0.059)$ \\
\hline & Slope - CN & $0.008(0.008)$ & $0.008(0.007)$ & $0.003(0.004)$ & $-0.006(0.006)$ \\
\hline & Slope - CI & $-0.000(0.019)$ & $0.014(0.020)$ & $-0.003(0.012)$ & $-0.013(0.016)$ \\
\hline & $F$-test & 0.68 & 0.48 & 1.63 & 0.68 \\
\hline$M S_{4} A 4 E-r s 670139$ & Intercept & $-0.045(0.048)$ & $-0.044(0.050)$ & $0.133(0.043)^{* *}$ & $0.055(0.046)$ \\
\hline & Slope - CN & $0.010(0.006)$ & $0.004(0.006)$ & $-0.001(0.003)$ & $-0.002(0.004)$ \\
\hline & Slope - CI & $0.006(0.016)$ & $0.013(0.017)$ & $0.005(0.010)$ & $0.014(0.013)$ \\
\hline & $F$-test kr & 0.44 & 0.65 & $4.06^{* *}$ & 2.35 \\
\hline$M S 4 A 6 A-r s 610932$ & Intercept & $-0.091(0.059)$ & $-0.029(0.062)$ & $0.042(0.053)$ & $0.035(0.057)$ \\
\hline & Slope - CN & $0.007(0.007)$ & $0.007(0.007)$ & $0.003(0.004)$ & $-0.003(0.005)$ \\
\hline & Slope - CI & $0.004(0.019)$ & $0.019(0.019)$ & $0.017(0.012)$ & $-0.009(0.015)$ \\
\hline & $F$-test & 0.83 & 0.59 & 1.28 & 0.26 \\
\hline PICALM-rs3851179 & Intercept & $0.029(0.065)$ & $0.010(0.069)$ & $0.049(0.059)$ & $0.041(0.064)$ \\
\hline & Slope - CN & $-0.005(0.008)$ & $0.002(0.008)$ & $0.002(0.005)$ & $0.006(0.006)$ \\
\hline & Slope - CI & $-0.021(0.023)$ & $0.006(0.023)$ & $-0.016(0.014)$ & $-0.007(0.018)$ \\
\hline & $F$-test & 0.37 & 0.06 & 0.74 & 0.72 \\
\hline SC-GRS & Intercept & $-0.008(0.009)$ & $0.003(0.010)$ & $0.008(0.008)$ & $0.009(0.009)$ \\
\hline
\end{tabular}




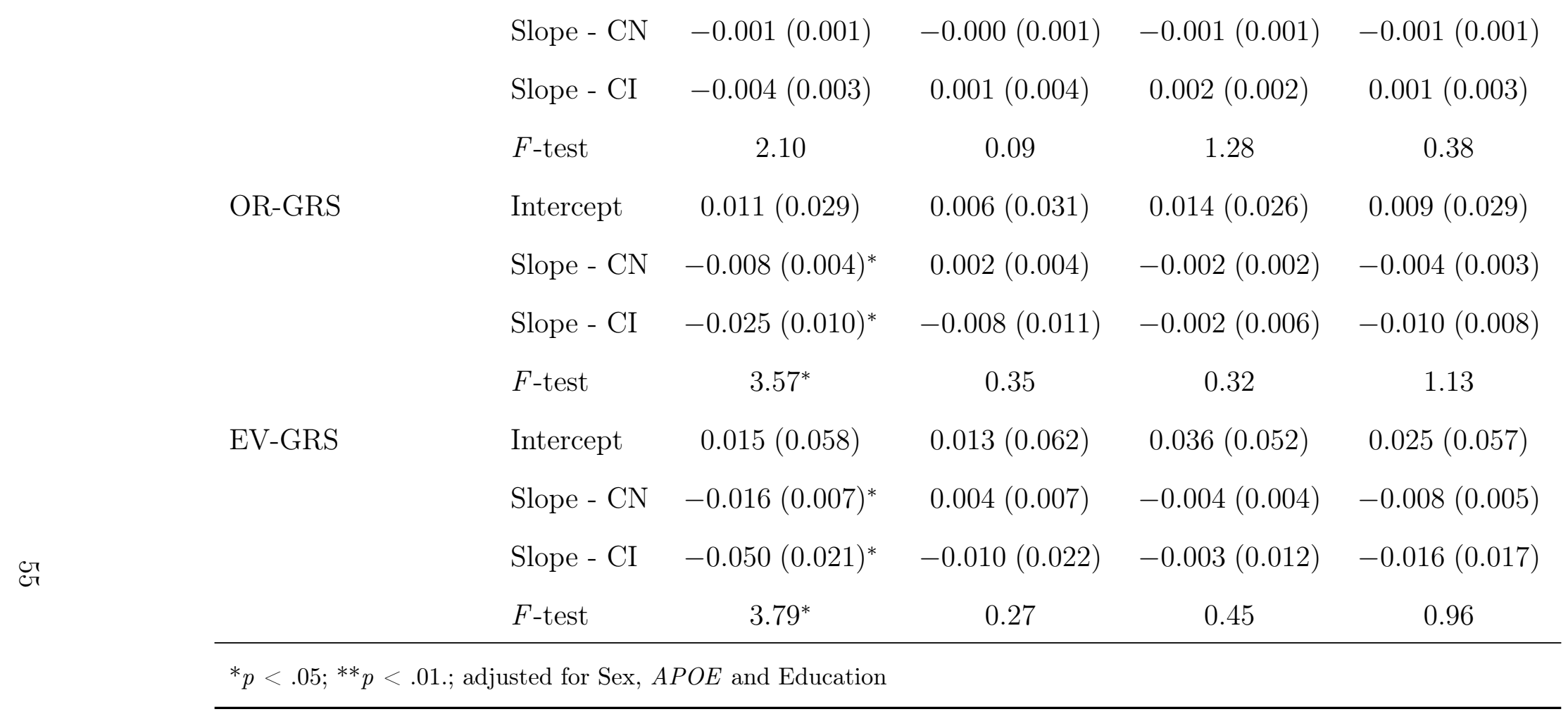


Table 45: Additional SNPs: Parameter estimates and model fit for SNP main effects according last diagnosis

\begin{tabular}{|c|c|c|c|c|c|}
\hline & & Episodic & Digits & Spot-the-Word & Symbol Digits \\
\hline & & Memeory & Backwards & & Modalities Test \\
\hline & & Estimate (SE) & Estimate (SE) & Estimate (SE) & Estimate (SE) \\
\hline \multirow[t]{4}{*}{$B D N F-$ rs6265 } & Intercept & $-0.016(0.049)$ & $-0.103(0.052)^{*}$ & $-0.008(0.045)$ & $0.042(0.048)$ \\
\hline & Slope - CN & $-0.005(0.006)$ & $-0.003(0.006)$ & $-0.004(0.003)$ & $-0.002(0.005)$ \\
\hline & Slope - CI & $-0.030(0.017)$ & $0.027(0.018)$ & $-0.022(0.011)^{*}$ & $0.013(0.014)$ \\
\hline & $F$-test & 1.58 & 2.60 & 2.11 & 0.60 \\
\hline \multirow[t]{4}{*}{$C E T P$-rs 5882} & Intercept & $-0.020(0.046)$ & $-0.068(0.048)$ & $-0.009(0.041)$ & $-0.001(0.045)$ \\
\hline & Slope - CN & $0.002(0.006)$ & $0.010(0.006)$ & $-0.004(0.003)$ & $0.001(0.004)$ \\
\hline & Slope - CI & $-0.029(0.016)$ & $0.000(0.017)$ & $-0.024(0.010)^{*}$ & $-0.013(0.013)$ \\
\hline & $F$-test & 1.22 & 1.19 & 2.49 & 0.33 \\
\hline \multirow[t]{4}{*}{$C O M T$-rs4680 } & Intercept & $0.104(0.053)$ & $0.033(0.056)$ & $0.022(0.048)$ & $-0.016(0.052)$ \\
\hline & Slope - CN & $-0.015(0.007)^{*}$ & $-0.004(0.007)$ & $0.004(0.004)$ & $-0.008(0.005)$ \\
\hline & Slope - CI & $-0.009(0.017)$ & $-0.008(0.018)$ & $0.001(0.011)$ & $-0.005(0.014)$ \\
\hline & $F$-test & 1.97 & 0.21 & 0.61 & 1.20 \\
\hline \multirow[t]{3}{*}{ CTNNBL1-rs6125962 } & Intercept & $-0.059(0.070)$ & $-0.021(0.074)$ & $0.001(0.064)$ & $-0.089(0.068)$ \\
\hline & Slope - CN & $0.015(0.009)$ & $0.006(0.009)$ & $-0.005(0.005)$ & $0.002(0.007)$ \\
\hline & Slope - CI & $0.055(0.022)^{*}$ & $0.058(0.022)^{* *}$ & $0.030(0.014)^{*}$ & $0.032(0.017)$ \\
\hline
\end{tabular}




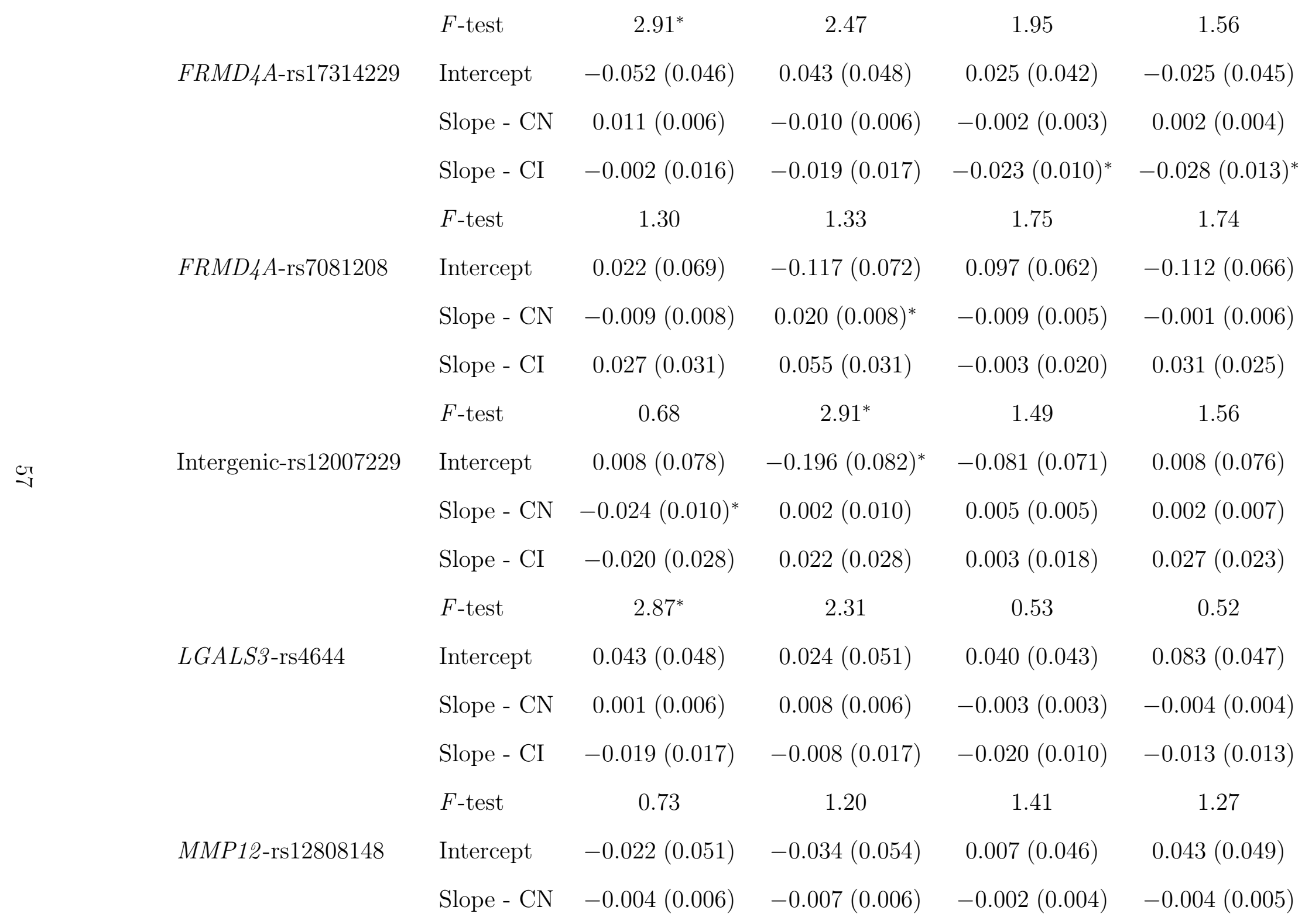




\begin{tabular}{|c|c|c|c|c|c|}
\hline & Slope - CI & $0.006(0.018)$ & $-0.011(0.018)$ & $0.020(0.011)$ & $0.001(0.014)$ \\
\hline & $F$-test & 0.45 & 1.11 & 1.09 & 0.34 \\
\hline MTHFD1L-rs11754661 & Intercept & $0.063(0.062)$ & $-0.047(0.065)$ & $0.009(0.056)$ & $0.072(0.060)$ \\
\hline & Slope - CN & $0.002(0.008)$ & $-0.003(0.008)$ & $-0.004(0.004)$ & $-0.004(0.006)$ \\
\hline & Slope - CI & $-0.028(0.020)$ & $0.014(0.020)$ & $-0.024(0.012)^{*}$ & $-0.008(0.016)$ \\
\hline & $F$-test & 1.13 & 0.51 & 1.52 & 0.55 \\
\hline PAICS-rs11549976 & Intercept & $0.042(0.071)$ & $-0.057(0.075)$ & $-0.078(0.065)$ & $-0.090(0.070)$ \\
\hline & Slope - CN & $-0.011(0.009)$ & $0.005(0.009)$ & $0.002(0.005)$ & $0.003(0.007)$ \\
\hline & Slope - CI & $0.026(0.026)$ & $0.018(0.027)$ & $0.037(0.016)^{*}$ & $0.003(0.021)$ \\
\hline & $F$-test & 0.89 & 0.31 & 2.16 & 0.56 \\
\hline PDE $7 A$-rs 10808746 & Intercept & $-0.021(0.049)$ & $-0.109(0.052)^{*}$ & $-0.019(0.045)$ & $-0.049(0.048)$ \\
\hline & Slope - CN & $0.003(0.006)$ & $0.010(0.006)$ & $0.000(0.003)$ & $0.008(0.005)$ \\
\hline & Slope - CI & $0.033(0.018)$ & $0.004(0.019)$ & $-0.008(0.012)$ & $0.036(0.015)^{*}$ \\
\hline & $F$-test & 1.12 & 1.70 & 0.27 & $3.06^{*}$ \\
\hline SNTG1-rs16914781 & Intercept & $0.062(0.049)$ & $0.036(0.052)$ & $-0.029(0.045)$ & $0.008(0.048)$ \\
\hline & Slope - CN & $-0.007(0.006)$ & $-0.002(0.006)$ & $-0.003(0.003)$ & $-0.001(0.005)$ \\
\hline & Slope - CI & $-0.023(0.018)$ & $-0.020(0.018)$ & $0.006(0.011)$ & $-0.012(0.014)$ \\
\hline & $F$-test & 1.02 & 0.48 & 0.64 & 0.26 \\
\hline SORL1-rs668387 & Intercept & $0.034(0.050)$ & $0.010(0.053)$ & $0.091(0.045)^{*}$ & $0.017(0.049)$ \\
\hline
\end{tabular}




\begin{tabular}{|c|c|c|c|c|c|}
\hline & Slope - CN & $-0.008(0.006)$ & $0.004(0.006)$ & $-0.004(0.004)$ & $0.003(0.005)$ \\
\hline & Slope - CI & $-0.010(0.017)$ & $-0.002(0.017)$ & $-0.017(0.011)$ & $0.000(0.014)$ \\
\hline & $F$-test & 0.57 & 0.27 & 2.02 & 0.27 \\
\hline SPON1-rs11023139 & Intercept & $0.084(0.075)$ & $-0.093(0.079)$ & $-0.096(0.068)$ & $0.033(0.074)$ \\
\hline & Slope - CN & $-0.012(0.009)$ & $0.006(0.009)$ & $0.004(0.005)$ & $-0.005(0.007)$ \\
\hline & Slope - CI & $-0.009(0.028)$ & $0.023(0.028)$ & $-0.014(0.018)$ & $0.008(0.023)$ \\
\hline & $F$-test & 0.63 & 0.60 & 0.97 & 0.24 \\
\hline \multirow[t]{4}{*}{ rs3746319 } & Intercept & $0.025(0.050)$ & $0.097(0.053)$ & $0.096(0.045)^{*}$ & $0.056(0.049)$ \\
\hline & Slope - CN & $-0.005(0.006)$ & $0.004(0.006)$ & $0.001(0.003)$ & $0.002(0.005)$ \\
\hline & Slope - CI & $-0.018(0.019)$ & $0.004(0.019)$ & $-0.007(0.012)$ & $0.020(0.015)$ \\
\hline & $F$-test & 0.33 & 2.58 & 2.45 & 1.29 \\
\hline
\end{tabular}




\section{References}

Baune, B.T., Konrad, C., Grotegerd, D., Suslow, T., Ohrmann, P., Bauer, J., Arolt, V., Heindel, W., Domschke, K., Schoning, S., Rauch, A.V., Sehlmeyer, C., Kugel, H., Dannlowski, U., 2012. Tumor necrosis factor gene variation predicts hippocampus volume in healthy individuals. Biological Psychiatry 72, 655-662.

Bayer, J., Rune, G., Kutsche, K., Schwarze, U., Kalisch, R., Büchel, C., Sommer, T., 2013. Estrogen and the male hippocampus: Genetic variation in the aromatase gene predicting serum estrogen is associated with hippocampal gray matter volume in men. Hippocampus 23, 117-121.

Beecham, G.W., Martin, E.R., Li, Y.J., Slifer, M.A., Gilbert, J.R., Haines, J.L.,

Pericak-Vance, M.A., 2009. Genome-wide Association Study Implicates a Chromosome 12 Risk Locus for Late-Onset Alzheimer Disease. American journal of human genetics $84,35-43$.

Bis, J.C., DeCarli, C., Smith, A.V., van der Lijn, F., Crivello, F., Fornage, M., Debette, S., Shulman, J.M., Schmidt, H., Srikanth, V., Schuur, M., Yu, L., Choi, S.H., Sigurdsson, S., Verhaaren, B.F.J., DeStefano, A.L., Lambert, J.C., Jack, C.R.J., Struchalin, M., Stankovich, J., Ibrahim-Verbaas, C.A., Fleischman, D., Zijdenbos, A., den Heijer, T., Mazoyer, B., Coker, L.H., Enzinger, C., Danoy, P., Amin, N., Arfanakis, K., van Buchem, M.A., de Bruijn, R.F.A.G., Beiser, A., Dufouil, C., Huang, J., Cavalieri, M., Thomson, R., Niessen, W.J., Chibnik, L.B., Gislason, G.K., Hofman, A., Pikula, A., Amouyel, P., Freeman, K.B., Phan, T.G., Oostra, B.A., Stein, J.L., Medland, S.E., Vasquez, A.A., Hibar, D.P., Wright, M.J., Franke, B., Martin, N.G., Thompson, P.M., Nalls, M.A., Uitterlinden, A.G., Au, R., Elbaz, A., Beare, R.J., van Swieten, J.C., López, O.L., Harris, T.B., Chouraki, V., Breteler, M.M.B., De Jager, P.L., Becker, J.T., Vernooij, M.W., Knopman, D., Fazekas, F., Wolf, P.A., van der Lugt, A., Gudnason, V., Longstreth, W.T.J., Brown, M.A., Bennett, D.A., van Duijn, C.M., Mosley, T.H., 
Schmidt, R., Tzourio, C., Launer, L.J., Ikram, M.A., Seshadri, S., 2012. Common variants at 12q14 and 12q24 are associated with hippocampal volume. Nat Genet 44, $545-551$.

De Jager, P.L., Shulman, J.M., Chibnik, L.B., Keenan, B.T., Raj, T., Wilson, R.S., Yu, L., Leurgans, S.E., Tran, D., Aubin, C., Anderson, C.D., Biffi, A., Corneveaux, J.J., Huentelman, M.J., Rosand, J., Daly, M.J., Myers, A.J., Reiman, E.M., Bennett, D.A., Evans, D.A., 2012. A genome-wide scan for common variants affecting the rate of age-related cognitive decline. Neurobiology of Aging 33, 1017.e1-1017.e15.

Fornage, M., Debette, S., Bis, J.C., Schmidt, H., Ikram, M.A., Dufouil, C., Sigurdsson, S., Lumley, T., DeStefano, A.L., Fazekas, F., Vrooman, H.A., Shibata, D.K., Maillard, P., Zijdenbos, A., Smith, A.V., Gudnason, H., de Boer, R., Cushman, M., Mazoyer, B., Heiss, G., Vernooij, M.W., Enzinger, C., Glazer, N.L., Beiser, A., Knopman, D.S., Cavalieri, M., Niessen, W.J., Harris, T.B., Petrovic, K., Lopez, O.L., Au, R., Lambert, J.C., Hofman, A., Gottesman, R.F., Garcia, M., Heckbert, S.R., Atwood, L.D., Catellier, D.J., Uitterlinden, A.G., Yang, Q., Smith, N.L., Aspelund, T., Romero, J.R., Rice, K., Taylor, K.D., Nalls, M.A., Rotter, J.I., Sharrett, R., van Duijn, C.M., Amouyel, P., Wolf, P.A., Gudnason, V., van der Lugt, A., Boerwinkle, E., Psaty, B.M., Seshadri, S., Tzourio, C., Breteler, M.M.B., Mosley, T.H., Schmidt, R., Longstreth, W.T., DeCarli, C., Launer, L.J., 2011. Genome-wide association studies of cerebral white matter lesion burden. Annals of Neurology 69, 928-939.

Harold, D., Abraham, R., Hollingworth, P., Sims, R., Gerrish, A., Hamshere, M.L., Pahwa, J.S., Moskvina, V., Dowzell, K., Williams, A., Jones, N., Thomas, C., Stretton, A., Morgan, A.R., Lovestone, S., Powell, J., Proitsi, P., Lupton, M.K., Brayne, C., Rubinsztein, D.C., Gill, M., Lawlor, B., Lynch, A., Morgan, K., Brown, K.S., Passmore, P.A., Craig, D., McGuinness, B., Todd, S., Holmes, C., Mann, D., Smith, A.D., Love, S., Kehoe, P.G., Hardy, J., Mead, S., Fox, N., Rossor, M., Collinge, J., Maier, W., 
Jessen, F., Schurmann, B., van den Bussche, H., Heuser, I., Kornhuber, J., Wiltfang, J., Dichgans, M., Frolich, L., Hampel, H., Hull, M., Rujescu, D., Goate, A.M., Kauwe, J.S.K., Cruchaga, C., Nowotny, P., Morris, J.C., Mayo, K., Sleegers, K., Bettens, K., Engelborghs, S., De yn, P.P., Van Broeckhoven, C., Livingston, G., Bass, N.J., Gurling, H., McQuillin, A., Gwilliam, R., Deloukas, P., Al-Chalabi, A., Shaw, C.E., Tsolaki, M., Singleton, A.B., Guerreiro, R., Muhleisen, T.W., Nothen, M.M., Moebus, S., Jockel, K.H., Klopp, N., Wichmann, H.E., Carrasquillo, M.M., Pankratz, V.S., Younkin, S.G., Holmans, P.A., O’Donovan, M., Owen, M.J., Williams, J., 2009. Genome-wide association study identifies variants at CLU and PICALM associated with Alzheimer's disease. Nat Genet 41, 1088-1093.

Harris, S.E., Fox, H., Wright, A.F., Hayward, C., Starr, J.M., Whalley, L.J., Deary, I.J., 2006. The brain-derived neurotrophic factor Val66Met polymorphism is associated with age-related change in reasoning skills. Molecular Psychiatry 11, 505-513.

Harris, S.E., Wright, A.F., Hayward, C., Starr, J.M., Whalley, L.J., Deary, I.J., 2005.

The functional COMT polymorphism, Val 158 Met, is associated with logical memory and the personality trait intellect/imagination in a cohort of healthy 79 year olds. Neuroscience Letters 385, 1-6.

Ho, A.J., Stein, J.L., Hua, X., Lee, S., Hibar, D.P., Leow, A.D., Dinov, I.D., Toga, A.W., Saykin, A.J., Shen, L., Foroud, T., Pankratz, N., Huentelman, M.J., Craig, D.W., Gerber, J.D., Allen, A.N., Corneveaux, J.J., Stephan, D.A., DeCarli, C.S., DeChairo, B.M., Potkin, S.G., Jack, C.R., Weiner, M.W., Raji, C.A., Lopez, O.L., Becker, J.T., Carmichael, O.T., Thompson, P.M., Initiative, t.A.D.N., 2010. A commonly carried allele of the obesity-related FTO gene is associated with reduced brain volume in the healthy elderly. Proceedings of the National Academy of Sciences 107, 8404-8409.

Hollingworth, P., Harold, D., Sims, R., Gerrish, A., Lambert, J.C., Carrasquillo, M.M., Abraham, R., Hamshere, M.L., Pahwa, J.S., Moskvina, V., Dowzell, K., Jones, N., 
Stretton, A., Thomas, C., Richards, A., Ivanov, D., Widdowson, C., Chapman, J., Lovestone, S., Powell, J., Proitsi, P., Lupton, M.K., Brayne, C., Rubinsztein, D.C., Gill, M., Lawlor, B., Lynch, A., Brown, K.S., Passmore, P.A., Craig, D., McGuinness, B., Todd, S., Holmes, C., Mann, D., Smith, A.D., Beaumont, H., Warden, D., Wilcock, G., Love, S., Kehoe, P.G., Hooper, N.M., Vardy, E.R.L.C., Hardy, J., Mead, S., Fox, N.C., Rossor, M., Collinge, J., Maier, W., Jessen, F., Ruther, E., Schurmann, B., Heun, R., Kolsch, H., van den Bussche, H., Heuser, I., Kornhuber, J., Wiltfang, J., Dichgans, M., Frolich, L., Hampel, H., Gallacher, J., Hull, M., Rujescu, D., Giegling, I., Goate, A.M., Kauwe, J.S.K., Cruchaga, C., Nowotny, P., Morris, J.C., Mayo, K., Sleegers, K., Bettens, K., Engelborghs, S., De yn, P.P., Van Broeckhoven, C., Livingston, G., Bass, N.J., Gurling, H., McQuillin, A., Gwilliam, R., Deloukas, P., Al-Chalabi, A., Shaw, C.E., Tsolaki, M., Singleton, A.B., Guerreiro, R., Muhleisen, T.W., Nothen, M.M., Moebus, S., Jockel, K.H., Klopp, N., Wichmann, H.E., Pankratz, V.S., Sando, S.B., Aasly, J.O., Barcikowska, M., Wszolek, Z.K., Dickson, D.W., Graff-Radford, N.R., Petersen, R.C., van Duijn, C.M., Breteler, M.M.B., Ikram, M.A., DeStefano, A.L., Fitzpatrick, A.L., Lopez, O., Launer, L.J., Seshadri, S., Berr, C., Campion, D., Epelbaum, J., Dartigues, J.F., Tzourio, C., Alperovitch, A., Lathrop, M., Feulner, T.M., Friedrich, P., Riehle, C., Krawczak, M., Schreiber, S., Mayhaus, M., Nicolhaus, S., Wagenpfeil, S., Steinberg, S., Stefansson, H., Stefansson, K., Snaedal, J., Bjornsson, S., Jonsson, P.V., Chouraki, V., Genier-Boley, B., Hiltunen, M., Soininen, H., Combarros, O., Zelenika, D., Delepine, M., Bullido, M.J., Pasquier, F., Mateo, I., Frank-García, A., Porcellini, E., Hanon, O., Coto, E., Alvarez, V., Bosco, P., Siciliano, G., Mancuso, M., Panza, F., Solfrizzi, V., Nacmias, B., Sorbi, S., Bossu, P., Piccardi, P., Arosio, B., Annoni, G., Seripa, D., Pilotto, A., Scarpini, E., Galimberti, D., Brice, A., Hannequin, D., Licastro, F., Jones, L., Holmans, P.A., Jonsson, T., Riemenschneider, M., Morgan, K., Younkin, S.G., Owen, M.J., O'Donovan, M., Amouyel, P., Williams, J., 2011. Common variants at ABCA7, MS4A6A/MS4A4E, EPHA1, CD33 and CD2AP are associated with Alzheimer's disease. 
Nat Genet 43, 429-435.

Houlihan, L.M., Harris, S.E., Luciano, M., Gow, A.J., Starr, J.M., Visscher, P.M., Deary, I.J., 2009. Replication study of candidate genes for cognitive abilities: the Lothian Birth Cohort 1936. Genes, Brain and Behavior 8, 238-247.

Izaks, G.J., van der Knaap, A.M., Gansevoort, R.T., Navis, G., Slaets, J.P.J., Dullaart, R.P.F., 2012. Cholesteryl Ester Transfer Protein (CETP) genotype and cognitive function in persons aged 35 years or older. Neurobiology of Aging 33, 1851.e7-1851.e16.

Jahanshad, N., Rajagopalan, P., Hua, X., Hibar, D.P., Nir, T.M., Toga, A.W., Jack, C.R., Saykin, A.J., Green, R.C., Weiner, M.W., Medland, S.E., Montgomery, G.W., Hansell, N.K., McMahon, K.L., de Zubicaray, G.I., Martin, N.G., Wright, M.J., Thompson, P.M., Initiative, t.A.D.N., 2013. Genome-wide scan of healthy human connectome discovers SPON1 gene variant influencing dementia severity. Proceedings of the National Academy of Sciences 110, 4768-4773.

Johnson, A.D., Newton-Cheh, C., Chasman, D.I., Ehret, G.B., Johnson, T., Rose, L., Rice, K., Verwoert, G.C., Launer, L.J., Gudnason, V., Larson, M.G., Chakravarti, A., Psaty, B.M., Caulfield, M., van Duijn, C.M., Ridker, P.M., Munroe, P.B., Levy, D., 2011a. Association of hypertension drug target genes with blood pressure and hypertension in 86,588 individuals. Hypertension 57, 903-910.

Johnson, T., Gaunt, T.R., Newhouse, S.J., Padmanabhan, S., Tomaszewski, M., Kumari, M., Morris, R.W., Tzoulaki, I., O’Brien, E.T., Poulter, N.R., Sever, P., Shields, D.C., Thom, S., Wannamethee, S.G., Whincup, P.H., Brown, M.J., Connell, J.M., Dobson, R.J., Howard, P.J., Mein, C.A., Onipinla, A., Shaw-Hawkins, S., Zhang, Y., Davey Smith, G., Day, I.N.M., Lawlor, D.A., Goodall, A.H., Fowkes, F.G., Abecasis, G.R., Elliott, P., Gateva, V., Braund, P.S., Burton, P.R., Nelson, C.P., Tobin, M.D., van der Harst, P., Glorioso, N., Neuvrith, H., Salvi, E., Staessen, J.A., Stucchi, A., 
Devos, N., Jeunemaitre, X., Plouin, P.F., Tichet, J., Juhanson, P., Org, E., Putku, M., Sober, S., Veldre, G., Viigimaa, M., Levinsson, A., Rosengren, A., Thelle, D.S., Hastie, C.E., Hedner, T., Lee, W.K., Melander, O., Wahlstrand, B., Hardy, R., Wong, A., Cooper, J.A., Palmen, J., Chen, L., Stewart, A.F.R., Wells, G.A., Westra, H.J., Wolfs, M.G.M., Clarke, R., Franzosi, M.G., Goel, A., Hamsten, A., Lathrop, M., Peden, J.F., Seedorf, U., Watkins, H., Ouwehand, W.H., Sambrook, J., Stephens, J., Casas, J.P., Drenos, F., Holmes, M.V., Kivimaki, M., Shah, S., Shah, T., Talmud, P.J., Whittaker, J., Wallace, C., Delles, C., Laan, M., Kuh, D., Humphries, S.E., Nyberg, F., Cusi, D., Roberts, R., Newton-Cheh, C., Franke, L., Stanton, A.V., Dominiczak, A.F., Farrall, M., Hingorani, A.D., Samani, N.J., Caulfield, M.J., Munroe, P.B., 2011b. Blood pressure loci identified with a gene-centric array. American Journal of Human Genetics 89, 688-700.

Joyner, A.H., J, C.R., Bloss, C.S., Bakken, T.E., Rimol, L.M., Melle, I., Agartz, I., Djurovic, S., Topol, E.J., Schork, N.J., Andreassen, O.A., Dale, A.M., 2009. A common MECP2 haplotype associates with reduced cortical surface area in humans in two independent populations. Proceedings of the National Academy of Sciences 106, $15483-15488$.

Kamboh, M.I., Demirci, F.Y., Wang, X., Minster, R.L., Carrasquillo, M.M., Pankratz, V.S., Younkin, S.G., Saykin, A.J., Jun, G., Baldwin, C., Logue, M.W., Buros, J., Farrer, L., Pericak-Vance, M.A., Haines, J.L., Sweet, R.A., Ganguli, M., Feingold, E., DeKosky, S.T., Lopez, O.L., Barmada, M.M., 2012. Genome-wide association study of Alzheimer's disease. Transl Psychiatry 2, e117.

Kohannim, O., Hibar, D.P., Jahanshad, N., Stein, J.L., Hua, X., Toga, A.W., Jack, C.R.J., Weiner, M.W., Thompson, P.M., 2012. PREDICTING TEMPORAL LOBE VOLUME ON MRI FROM GENOTYPES USING L(1)-L(2) REGULARIZED REGRESSION. Proceedings / IEEE International Symposium on Biomedical Imaging: from nano to macro. IEEE International Symposium on Biomedical Imaging , 1160-1163. 
Lambert, J.C., Grenier-Boley, B., Harold, D., Zelenika, D., Chouraki, V., Kamatani, Y., Sleegers, K., Ikram, M.A., Hiltunen, M., Reitz, C., Mateo, I., Feulner, T., Bullido, M., Galimberti, D., Concari, L., Alvarez, V., Sims, R., Gerrish, A., Chapman, J., Deniz-Naranjo, C., Solfrizzi, V., Sorbi, S., Arosio, B., Spalletta, G., Siciliano, G., Epelbaum, J., Hannequin, D., Dartigues, J.F., Tzourio, C., Berr, C., Schrijvers, E.M.C., Rogers, R., Tosto, G., Pasquier, F., Bettens, K., Van Cauwenberghe, C., Fratiglioni, L., Graff, C., Delepine, M., Ferri, R., Reynolds, C.A., Lannfelt, L., Ingelsson, M., Prince, J.A., Chillotti, C., Pilotto, A., Seripa, D., Boland, A., Mancuso, M., Bossu, P., Annoni, G., Nacmias, B., Bosco, P., Panza, F., Sanchez-Garcia, F., Del Zompo, M., Coto, E., Owen, M., O’Donovan, M., Valdivieso, F., Caffara, P., Scarpini, E., Combarros, O., Buee, L., Campion, D., Soininen, H., Breteler, M., Riemenschneider, M., Van Broeckhoven, C., Alperovitch, A., Lathrop, M., Tregouet, D.A., Williams, J., Amouyel, P., 2013. Genome-wide haplotype association study identifies the FRMD4A gene as a risk locus for Alzheimer's disease. Molecular Psychiatry 18, 461-470.

Lambert, J.C., Heath, S., Even, G., Campion, D., Sleegers, K., Hiltunen, M., Combarros, O., Zelenika, D., Bullido, M.J., Tavernier, B., Letenneur, L., Bettens, K., Berr, C., Pasquier, F., Fievet, N., Barberger-Gateau, P., Engelborghs, S., De yn, P., Mateo, I., Franck, A., Helisalmi, S., Porcellini, E., Hanon, O., de Pancorbo, M.M., Lendon, C., Dufouil, C., Jaillard, C., Leveillard, T., Alvarez, V., Bosco, P., Mancuso, M., Panza, F., Nacmias, B., Bossu, P., Piccardi, P., Annoni, G., Seripa, D., Galimberti, D., Hannequin, D., Licastro, F., Soininen, H., Ritchie, K., Blanche, H., Dartigues, J.F., Tzourio, C., Gut, I., Van Broeckhoven, C., Alperovitch, A., Lathrop, M., Amouyel, P., 2009. Genome-wide association study identifies variants at CLU and CR1 associated with Alzheimer's disease. Nat Genet 41, 1094-1099.

Levy, D., Ehret, G.B., Rice, K., Verwoert, G.C., Launer, L.J., Dehghan, A., Glazer, N.L., Morrison, A.C., Johnson, A.D., Aspelund, T., Aulchenko, Y., Lumley, T., Kottgen, A., Vasan, R.S., Rivadeneira, F., Eiriksdottir, G., Guo, X., Arking, D.E., Mitchell, G.F., 
Mattace-Raso, F.U.S., Smith, A.V., Taylor, K., Scharpf, R.B., Hwang, S.J., Sijbrands, E.J.G., Bis, J., Harris, T.B., Ganesh, S.K., O’Donnell, C.J., Hofman, A., Rotter, J.I., Coresh, J., Benjamin, E.J., Uitterlinden, A.G., Heiss, G., Fox, C.S., Witteman, J.C.M., Boerwinkle, E., Wang, T.J., Gudnason, V., Larson, M.G., Chakravarti, A., Psaty, B.M., van Duijn, C.M., 2009. Genome-wide association study of blood pressure and hypertension. Nat Genet 41, 677-687.

Li, J., Chen, C., Lei, X., Wang, Y., Chen, C., He, Q., Moyzis, R.K., Xue, G., Zhu, B., Cao, Z., Dong, Q., 2013. The NTSR1 gene modulates the association between hippocampal structure and working memory performance. NeuroImage 75, 79-86.

Mandelman, S.D., Grigorenko, E.L., 2012. BDNF Val66Met and cognition: all, none, or some? A meta-analysis of the genetic association. Genes, Brain and Behavior 11, $127-136$.

Markett, S., Reuter, M., Montag, C., Weber, B., 2013. The dopamine D2 receptor gene DRD2 and the nicotinic acetylcholine receptor gene CHRNA4 interact on striatal gray matter volume: evidence from a genetic imaging study. NeuroImage 64, 167-172.

Melville, S.A., Buros, J., Parrado, A.R., Vardarajan, B., Logue, M.W., Shen, L., Risacher, S.L., Kim, S., Jun, G., DeCarli, C., Lunetta, K.L., Baldwin, C.T., Saykin, A.J., Farrer, L.A., the Alzheimer's Disease Neuroimaging, I., 2012. Multiple loci influencing hippocampal degeneration identified by genome scan. Annals of Neurology 72, 65-75.

Naj, A.C., Jun, G., Beecham, G.W., Wang, L.S., Vardarajan, B.N., Buros, J., Gallins, P.J., Buxbaum, J.D., Jarvik, G.P., Crane, P.K., Larson, E.B., Bird, T.D., Boeve, B.F., Graff-Radford, N.R., De Jager, P.L., Evans, D., Schneider, J.A., Carrasquillo, M.M., Ertekin-Taner, N., Younkin, S.G., Cruchaga, C., Kauwe, J.S.K., Nowotny, P., Kramer, P., Hardy, J., Huentelman, M.J., Myers, A.J., Barmada, M.M., Demirci, F.Y., Baldwin, C.T., Green, R.C., Rogaeva, E., George-Hyslop, P.S., Arnold, S.E., Barber, R., Beach, 
T., Bigio, E.H., Bowen, J.D., Boxer, A., Burke, J.R., Cairns, N.J., Carlson, C.S., Carney, R.M., Carroll, S.L., Chui, H.C., Clark, D.G., Corneveaux, J., Cotman, C.W., Cummings, J.L., DeCarli, C., DeKosky, S.T., Diaz-Arrastia, R., Dick, M., Dickson, D.W., Ellis, W.G., Faber, K.M., Fallon, K.B., Farlow, M.R., Ferris, S., Frosch, M.P., Galasko, D.R., Ganguli, M., Gearing, M., Geschwind, D.H., Ghetti, B., Gilbert, J.R., Gilman, S., Giordani, B., Glass, J.D., Growdon, J.H., Hamilton, R.L., Harrell, L.E., Head, E., Honig, L.S., Hulette, C.M., Hyman, B.T., Jicha, G.A., Jin, L.W., Johnson, N., Karlawish, J., Karydas, A., Kaye, J.A., Kim, R., Koo, E.H., Kowall, N.W., Lah, J.J., Levey, A.I., Lieberman, A.P., Lopez, O.L., Mack, W.J., Marson, D.C., Martiniuk, F., Mash, D.C., Masliah, E., McCormick, W.C., McCurry, S.M., McDavid, A.N., McKee, A.C., Mesulam, M., Miller, B.L., Miller, C.A., Miller, J.W., Parisi, J.E., Perl, D.P., Peskind, E., Petersen, R.C., Poon, W.W., Quinn, J.F., Rajbhandary, R.A., Raskind, M., Reisberg, B., Ringman, J.M., Roberson, E.D., Rosenberg, R.N., Sano, M., Schneider, L.S., Seeley, W., Shelanski, M.L., Slifer, M.A., Smith, C.D., Sonnen, J.A., Spina, S., Stern, R.A., Tanzi, R.E., Trojanowski, J.Q., Troncoso, J.C., Van Deerlin, V.M., Vinters, H.V., Vonsattel, J.P., Weintraub, S., Welsh-Bohmer, K.A., Williamson, J., Woltjer, R.L., Cantwell, L.B., Dombroski, B.A., Beekly, D., Lunetta, K.L., Martin, E.R., Kamboh, M.I., Saykin, A.J., Reiman, E.M., Bennett, D.A., Morris, J.C., Montine, T.J., Goate, A.M., Blacker, D., Tsuang, D.W., Hakonarson, H., Kukull, W.A., Foroud, T.M., Haines, J.L., Mayeux, R., Pericak-Vance, M.A., Farrer, L.A., Schellenberg, G.D., 2011. Common variants at MS4A4/MS4A6E, CD2AP, CD33 and EPHA1 are associated with late-onset Alzheimer's disease. Nat Genet 43, 436-441.

Nho, K., Corneveaux, J.J., Kim, S., Lin, H., Risacher, S.L., Shen, L., Swaminathan, S., Ramanan, V.K., Liu, Y., Foroud, T., Inlow, M.H., Siniard, A.L., Reiman, R.A., Aisen, P.S., Petersen, R.C., Green, R.C., Jack, C.R., Weiner, M.W., Baldwin, C.T., Lunetta, K., Farrer, L.A., Furney, S.J., Lovestone, S., Simmons, A., Mecocci, P., Vellas, B., Tsolaki, M., Kloszewska, I., Soininen, H., McDonald, B.C., Farlow, M.R., Ghetti, B., 
Huentelman, M.J., Saykin, A.J., 2013. Whole-exome sequencing and imaging genetics identify functional variants for rate of change in hippocampal volume in mild cognitive impairment. Molecular Psychiatry 18, 781-787.

Papassotiropoulos, A., Stefanova, E., Vogler, C., Gschwind, L., Ackermann, S., Spalek, K., Rasch, B., Heck, A., Aerni, A., Hanser, E., Demougin, P., Huynh, K.D., Luechinger, R., Klarhofer, M., Novakovic, I., Kostic, V., Boesiger, P., Scheffler, K., de Quervain, D.J.F., 2013. A genome-wide survey and functional brain imaging study identify CTNNBL1 as a memory-related gene. Molecular Psychiatry 18, 255-263.

Rogaeva, E., Meng, Y., Lee, J.H., Gu, Y., Kawarai, T., Zou, F., Katayama, T., Baldwin, C.T., Cheng, R., Hasegawa, H., Chen, F., Shibata, N., Lunetta, K.L., Pardossi-Piquard, R., Bohm, C., Wakutani, Y., Cupples, L.A., Cuenco, K.T., Green, R.C., Pinessi, L., Rainero, I., Sorbi, S., Bruni, A., Duara, R., Friedland, R.P., Inzelberg, R., Hampe, W., Bujo, H., Song, Y.Q., Andersen, O.M., Willnow, T.E., Graff-Radford, N., Petersen, R.C., Dickson, D., Der, S.D., Fraser, P.E., Schmitt-Ulms, G., Younkin, S., Mayeux, R., Farrer, L.A., St George-Hyslop, P., 2007. The neuronal sortilin-related receptor SORL1 is genetically associated with Alzheimer disease. Nat Genet 39, 168-177.

Roussotte, F.F., Jahanshad, N., Hibar, D.P., Sowell, E.R., Kohannim, O., Barysheva, M., Hansell, N.K., McMahon, K.L., de Zubicaray, G.I., Montgomery, G.W., Martin, N.G., Wright, M.J., Toga, A.W., Jack, C.R.J., Weiner, M.W., Thompson, P.M., 2014. A commonly carried genetic variant in the delta opioid receptor gene, OPRD1, is associated with smaller regional brain volumes: replication in elderly and young populations. Human brain mapping 35, 1226-1236.

Salvi, E., Kutalik, Z., Glorioso, N., Benaglio, P., Frau, F., Kuznetsova, T., Arima, H., Hoggart, C., Tichet, J., Nikitin, Y.P., Conti, C., Seidlerova, J., Tikhonoff, V., Stolarz-Skrzypek, K., Johnson, T., Devos, N., Zagato, L., Guarrera, S., Zaninello, R., Calabria, A., Stancanelli, B., Troffa, C., Thijs, L., Rizzi, F., Simonova, G., Lupoli, 
S., Argiolas, G., Braga, D., D’Alessio, M.C., Ortu, M.F., Ricceri, F., Mercurio, M., Descombes, P., Marconi, M., Chalmers, J., Harrap, S., Filipovsky, J., Bochud, M., Iacoviello, L., Ellis, J., Stanton, A.V., Laan, M., Padmanabhan, S., Dominiczak, A.F., Samani, N.J., Melander, O., Jeunemaitre, X., Manunta, P., Shabo, A., Vineis, P., Cappuccio, F.P., Caulfield, M.J., Matullo, G., Rivolta, C., Munroe, P.B., Barlassina, C., Staessen, J.A., Beckmann, J.S., Cusi, D., 2012. Genomewide Association Study Using a High-Density Single Nucleotide Polymorphism Array and Case-Control Design Identifies a Novel Essential Hypertension Susceptibility Locus in the Promoter Region of Endothelial NO Synthase. Hypertension 59, 248-255.

Schrijvers, E.M.C., Schurmann, B., Koudstaal, P.J., van den Bussche, H., van Duijn, C.M., Hentschel, F., Heun, R., Hofman, A., Jessen, F., Kolsch, H., Kornhuber, J., Peters, O., Rivadeneira, F., Ruther, E., Uitterlinden, A.G., Riedel-Heller, S., Dichgans, M., Wiltfang, J., Maier, W., Breteler, M.M.B., Ikram, M.A., 2012. Genome-Wide Association Study of Vascular Dementia. Stroke 43, 315-319.

Seshadri S, F.A.L.I.M., et al, 2010. GEnome-wide analysis of genetic loci associated with alzheimer disease. JAMA 303, 1832-1840.

Sherva, R., Tripodis, Y., Bennett, D.A., Chibnik, L.B., Crane, P.K., De Jager, P.L., Farrer, L.A., Saykin, A.J., Shulman, J.M., Green, R.C., 2013. Genome-wide association study of the rate of cognitive decline in Alzheimer's disease. Alzheimer's \& Dementia .

Shulman, J.M., Chibnik, L.B., Aubin, C., Schneider, J.A., Bennett, D.A., De Jager, P.L., 2010. Intermediate Phenotypes Identify Divergent Pathways to Alzheimer's Disease. PLoS ONE 5, e11244.

Stein, J.L., Hibar, D.P., Madsen, S.K., Khamis, M., McMahon, K.L., de Zubicaray, G.I., Hansell, N.K., Montgomery, G.W., Martin, N.G., Wright, M.J., Saykin, A.J., Jack, C.R.J., Weiner, M.W., Toga, A.W., Thompson, P.M., 2011. Discovery and replication 
of dopamine-related gene effects on caudate volume in young and elderly populations ( $\mathrm{N}=1198)$ using genome-wide search. Molecular Psychiatry 16, 927-937.

Trompet, S., Jukema, W., Mooijaart, S.P., Ford, I., Stott, D.J., Westendorp, R.G.J., de Craen, A.J.M., 2012. Genetic variation in galectin-3 gene associates with cognitive function at old age. Neurobiology of Aging 33, 2232.e1-2232.e9.

Velez, J.I., Chandrasekharappa, S.C., Henao, E., Martinez, A.F., Harper, U., Jones, M., Solomon, B.D., Lopez, L., Garcia, G., Aguirre-Acevedo, D.C., Acosta-Baena, N., Correa, J.C., Lopera-Gomez, C.M., Jaramillo-Elorza, M.C., Rivera, D., Kosik, K.S., Schork, N.J., Swanson, J.M., Lopera, F., Arcos-Burgos, M., 2013. Pooling/bootstrap-based GWAS (pbGWAS) identifies new loci modifying the age of onset in PSEN1 p.Glu280Ala Alzheimer's disease. Molecular Psychiatry 18, 568-575. 